\title{
DESIGN, IMPLEMENTATION AND EXPERIMENTAL TESTING OF AN INERTIAL SENSOR SYSTEM TO QUANTIFY WING DEFLECTION
}

\author{
A Dissertation by \\ Georg Schirmer \\ Master of Science, Wichita State University, 2013 \\ Bachelor of Science in Aerospace Engineering, Wichita State University, 2011 \\ Submitted to the Department of Aerospace Engineering \\ and the faculty of the Graduate School of \\ Wichita State University \\ in partial fulfillment of \\ the requirements for the degree of \\ Doctor of Philosophy
}

May 2019 
(c) Copyright 2019 by Georg Schirmer

All Rights Reserved 


\section{DESIGN, IMPLEMENTATION AND EXPERIMENTAL TESTING OF AN INERTIAL SENSOR SYSTEM TO QUANTIFY WING DEFLECTION}

The following faculty members have examined the final copy of this dissertation for form and content, and recommend that it be accepted in partial fulfillment of the requirements for the degree of Doctor of Philosophy, with a major in Aerospace Engineering.

James Steck, Committee Chair

Animesh Chakravarthy, Committee Member

Brandon Buerge, Committee Member

Edwin Sawan, Committee Member

Ward Jewell, Committee Member

Accepted for the College of Engineering

Dennis Livesay, Dean

Accepted for the Graduate School

Kerry Wilks, Interim Dean 


\section{DEDICATION}

To my daughter Adelynn, whose inherent and ever-growing curiosity reminds me of the fuel for creativity and ingenuity as she motivates and inspires me every day. 


\section{ACKNOWLEDGEMENTS}

Every innovation begins with an idea, maybe a vision that evolves and manifests itself in the creative mind. However, the process of turning such a vision into reality is one that often necessitates the help and support of many others who deserve credit and recognition. I want to thank my father whose ingenuity has been a lifelong inspiration to me and who played an integral role in the early conceptual stages of this project, particularly during the initial preliminary testing. Throughout my time at Wichita State University I had the pleasure of working with many individuals who have positively influenced me in one way or another and I owe my sincere gratitude to them all. I would like to recognize the GAFL research group for the outstanding team work and particularly Alok Menon, who has been a true friend and tremendous help throughout every phase of the project. I want to give special thanks to committee chair Dr. James Steck, for his support, guidance, and encouragement during this research. I also want to express my appreciation to committee members Dr. Buerge, Dr. Chakravarthy, Dr. Jewell and Dr. Sawan for their willingness to devote their time and effort and provide me with invaluable input. I would like to give thanks to Dr. Suresh Keshavanarayana and his graduate student Dmitrii Ivanov for the help and allowing me to use the WSU Flight Structures Lab and equipment as well as NIAR, John Laffen and his staff for giving me the opportunity to test in the WSU Walter Beech Wind Tunnel. Finally, I want to thank my wife for her patience, her sacrifices and her support throughout this journey. In the end I would not have accomplished this without the help of many others. 


\begin{abstract}
This research investigates a novel approach of using inertial micro-electromechanical sensors in combination with a Kalman state estimator to quantify wing deflection of a highly flexible morphing wing. Motivated by NASA's and Boeing's collaborative research into multiobjective control of a highly flexible morphing wing, the work described herein illustrates the development and implementation of wing shape output feedback part of such a system. Equations for prediction and measurement are developed to estimate bending and torsion. Initially a static structural model serves as a means to construct deflection measurements using sensor data. System inputs are obtained using 3-axis accelerometers and gyroscopes. Sensor fusion of accelerometer and gyroscopic data is discussed. The system is expanded to incorporate a structural dynamics finite element model for the prediction and advanced inertial measurement unit motion processors to take measurements. Sensor analysis is performed to determine sensor specific performance characteristics which are used for the state estimator design. Extensive bench and wind tunnel testing of the final system design was conducted. The implementation, static and dynamic deflection testing and results of the system design are discussed. Future work is proposed and includes the development of a more advanced structural FEM model and the use of higher accuracy sensors units.
\end{abstract}




\section{TABLE OF CONTENTS}

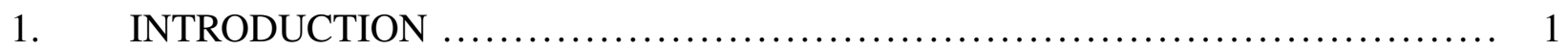

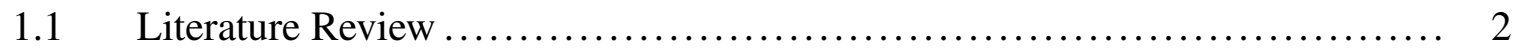

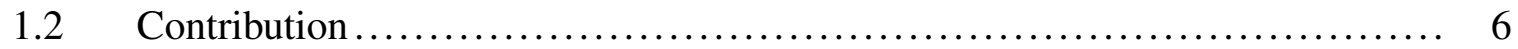

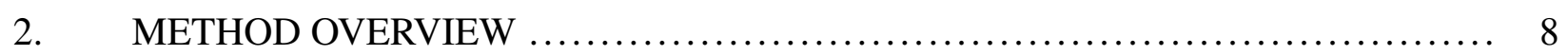

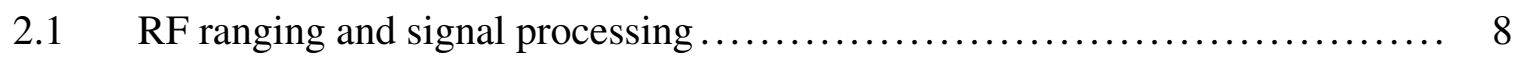

$2.1 .1 \quad$ Interferometry Approach (Amplitude) $\ldots \ldots \ldots \ldots \ldots \ldots \ldots \ldots \ldots \ldots . \ldots \ldots$

2.1.2 $\quad$ Pulse width modulation using a signal multiplier..................... 10

2.1 .3 Polarized signal attenuation approach $\ldots \ldots \ldots \ldots \ldots \ldots \ldots \ldots \ldots \ldots \ldots \ldots \ldots \ldots \ldots$

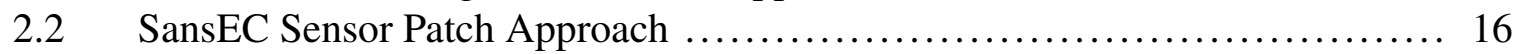

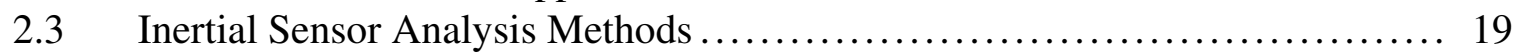

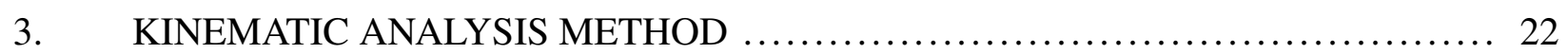

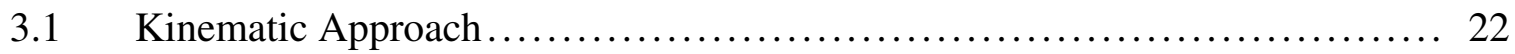

3.1.1 Kalman Filter and Sensor Fusion of kinematic and static sensor data. ... 24

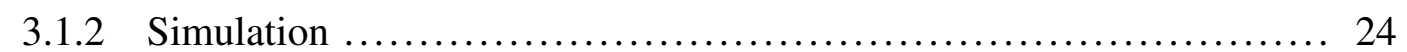

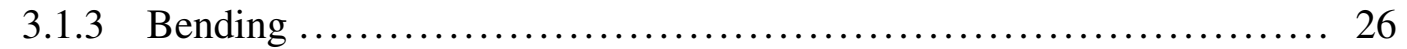

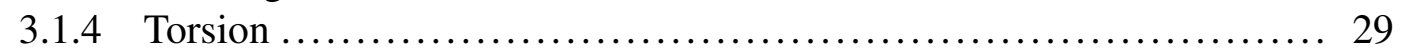

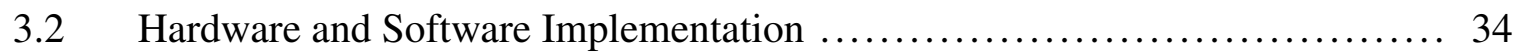

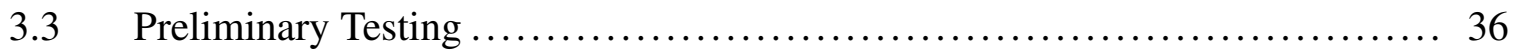

4. RESULTS AND DISCUSSION - PART 1: KINEMATIC APPROACH .............. 39

$4.1 \quad$ Position tracking using only accelerometer data $\ldots \ldots \ldots \ldots \ldots \ldots \ldots \ldots \ldots \ldots \ldots$

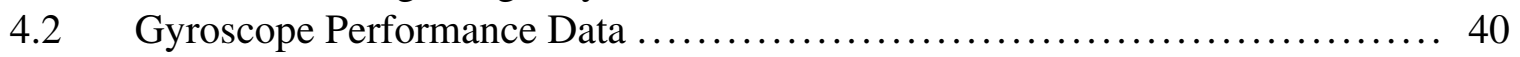

$4.3 \quad$ Kinematic Approach with Kalman Filter Implementation.................... 42

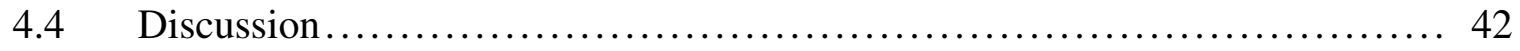

$4.4 .1 \quad$ Limitations of the Kinematic Approach........................... 43

5. STRUCTURAL DYNAMICS ANALYSIS METHOD $\ldots \ldots \ldots \ldots \ldots \ldots \ldots \ldots \ldots \ldots \ldots$

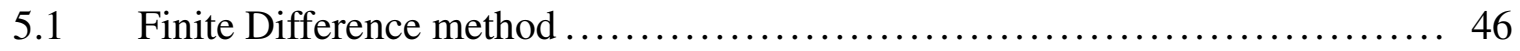

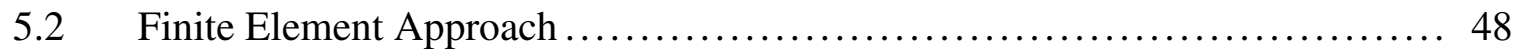

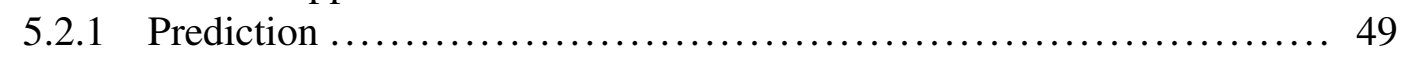

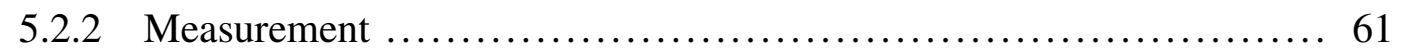

$5.2 .3 \quad$ Kalman Filter applied to FEM prediction ........................ 62 
TABLE OF CONTENTS (continued)

Chapter

Page

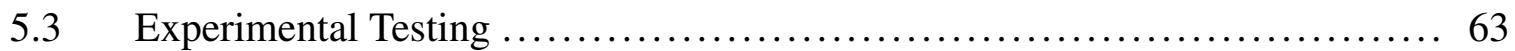

$5.3 .1 \quad$ Structural Dynamic Bench Testing .............................. 63

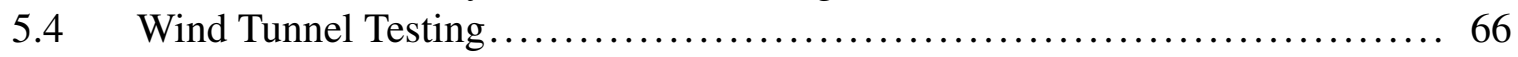

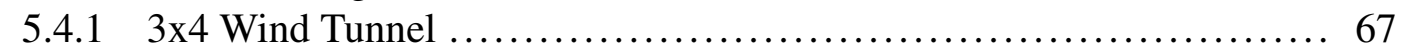

6. RESULTS AND DISCUSSION - PART 2: FINITE ELEMENT APPROACH. ........ 72

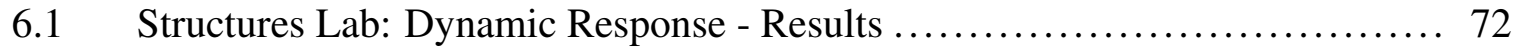

$6.1 .1 \quad$ Free Vibration, Non-Zero, Bending, No Twist Initial Condition........ 72

6.1.2 Free Vibration with complex S-shape Bending Initial condition......... 77

6.1 .3 Free Vibration with combined bending and twist initial condition ...... 81

$6.2 \quad$ Wind Tunnel: Dynamic Response - Results ............................ 84

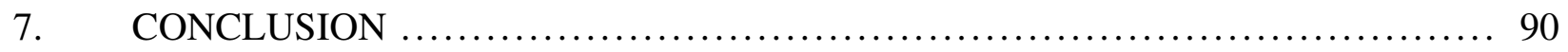

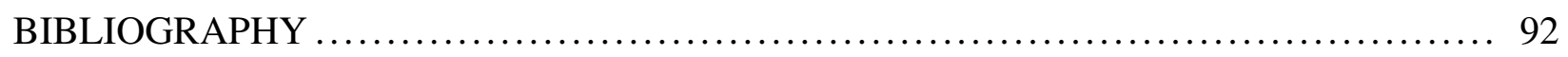

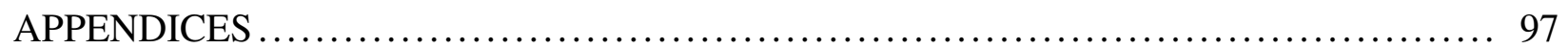

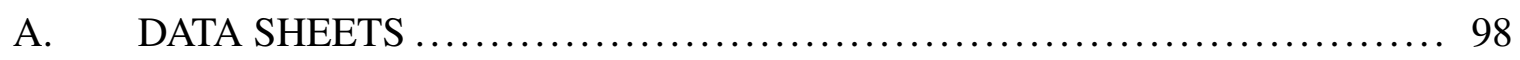




\section{LIST OF FIGURES}

Figure $\quad$ Page

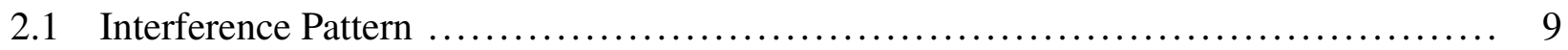

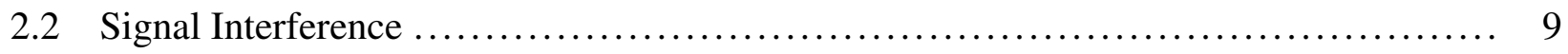

2.3 Conceptual graphic of pulse width modulation $\ldots \ldots \ldots \ldots \ldots \ldots \ldots \ldots \ldots \ldots \ldots \ldots \ldots, 11$

2.4 Impulse arrives with a delay of $1 / 3$ pulse width $\ldots \ldots \ldots \ldots \ldots \ldots \ldots \ldots \ldots \ldots \ldots \ldots \ldots$

2.5 Impulse arrives with a delay of $2 / 3$ pulse width $\ldots \ldots \ldots \ldots \ldots \ldots \ldots \ldots \ldots \ldots \ldots \ldots \ldots \ldots$

2.6 Conceptual Dipole Installation $\ldots \ldots \ldots \ldots \ldots \ldots \ldots \ldots \ldots \ldots \ldots \ldots \ldots \ldots \ldots \ldots \ldots \ldots \ldots \ldots$

$2.7 \quad$ Dipole directional signal attenuation $\ldots \ldots \ldots \ldots \ldots \ldots \ldots \ldots \ldots \ldots \ldots \ldots \ldots \ldots \ldots \ldots$

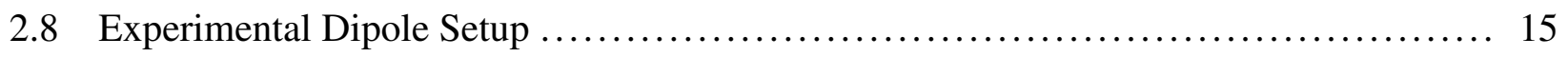

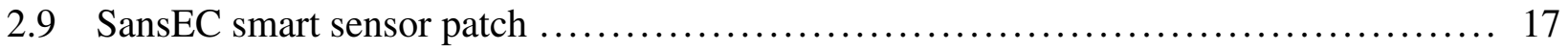

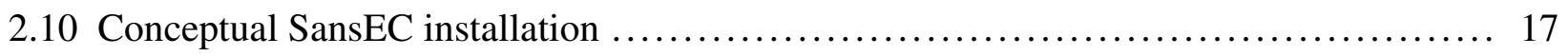

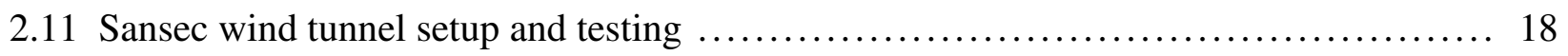

2.12 Frequency shift as result of deflection $\ldots \ldots \ldots \ldots \ldots \ldots \ldots \ldots \ldots \ldots \ldots \ldots \ldots \ldots \ldots \ldots \ldots$

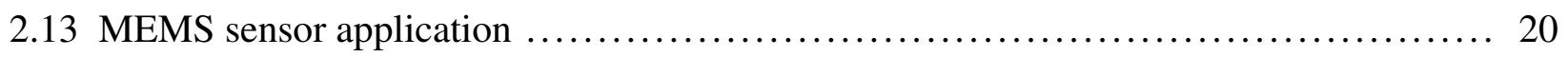

3.1 Simulation of Kalman Filter State Estimator $\ldots \ldots \ldots \ldots \ldots \ldots \ldots \ldots \ldots \ldots \ldots \ldots \ldots$

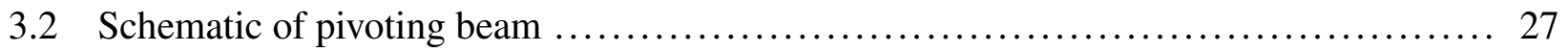

$3.3 \quad$ Wing deflection measurement using single accelerometer $\ldots \ldots \ldots \ldots \ldots \ldots \ldots \ldots \ldots \ldots 29$

3.4 Torsion Measurement with Accelerometer and Gyro $\ldots \ldots \ldots \ldots \ldots \ldots \ldots \ldots \ldots \ldots \ldots \ldots$

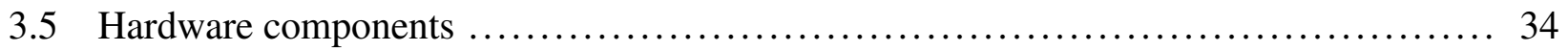

$3.6 \quad$ Sensor close-up and tear down (courtesy of Adafruit Inc. and Chipworks Inc............ 35

3.7 Pivoting beam with single 3 -axis accelerometer $\ldots \ldots \ldots \ldots \ldots \ldots \ldots \ldots \ldots \ldots \ldots \ldots \ldots$ 


\section{LIST OF FIGURES (continued)}

Figure $\quad$ Page

3.8 Torsion Measurement with Accelerometer and Gyro $\ldots \ldots \ldots \ldots \ldots \ldots \ldots \ldots \ldots \ldots \ldots . \ldots . \ldots . \ldots . \ldots$

4.1 Displacement based on integrating raw acceleration $\ldots \ldots \ldots \ldots \ldots \ldots \ldots \ldots \ldots \ldots . \ldots . \ldots$

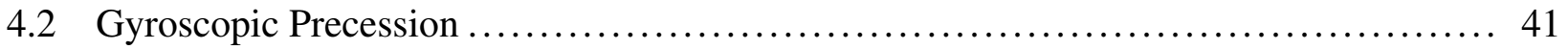

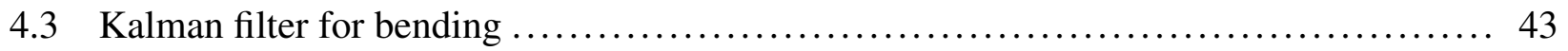

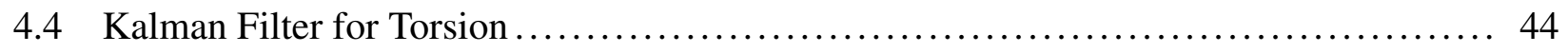

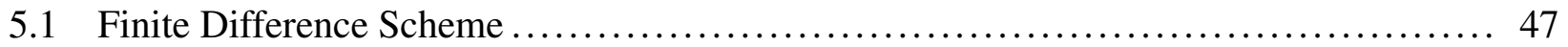

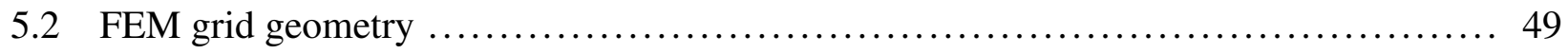

5.3 Illustration of sensors system arrangement $\ldots \ldots \ldots \ldots \ldots \ldots \ldots \ldots \ldots \ldots \ldots \ldots \ldots \ldots$

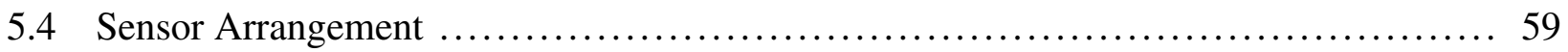

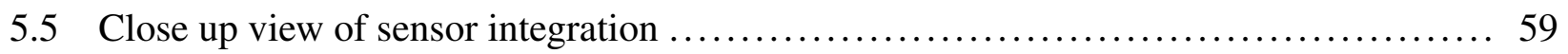

5.6 Experiment to obtain tune material properties $\ldots \ldots \ldots \ldots \ldots \ldots \ldots \ldots \ldots \ldots \ldots \ldots \ldots \ldots$. 61

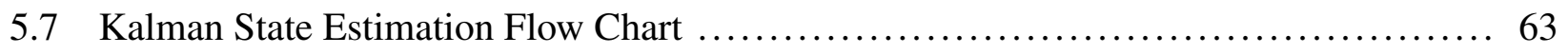

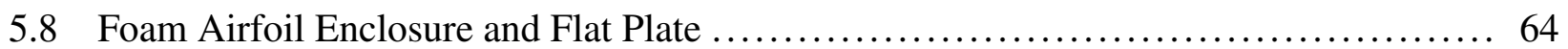

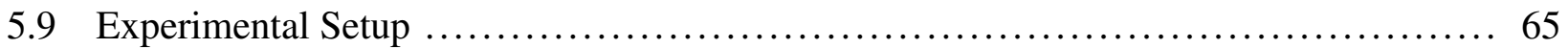

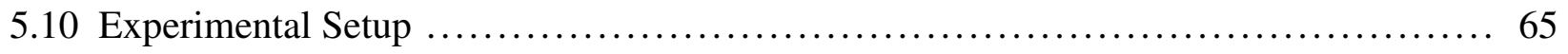

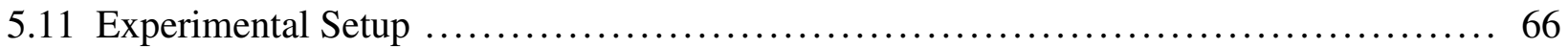

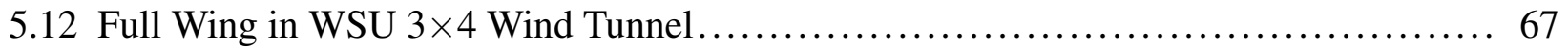

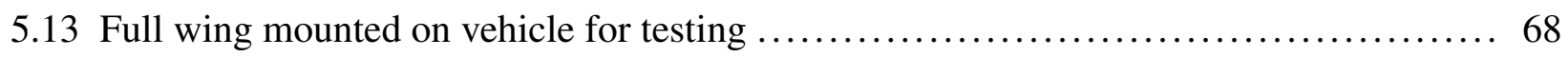

5.14 Optical Measurement System Installation $\ldots \ldots \ldots \ldots \ldots \ldots \ldots \ldots \ldots \ldots \ldots \ldots \ldots \ldots$

5.15 Half wing section PCB mounted in 7 x10 wind tunnel $\ldots \ldots \ldots \ldots \ldots \ldots \ldots \ldots \ldots \ldots$ 


\section{LIST OF FIGURES (continued)}

Figure $\quad$ Page

5.16 Half wing section PCB with foam structure mounted 7 x10 wind tunnel $\ldots \ldots \ldots \ldots \ldots . . . . .71$

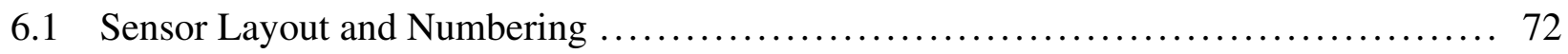

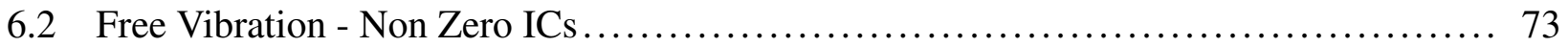

6.3 Free Vibration: Non-Zero Bending Initial Conditions, No Twist, Leading Edge Sensor

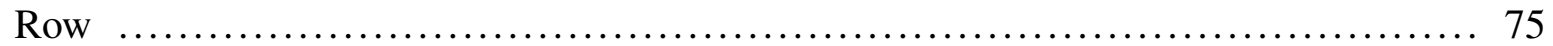

6.4 Free Vibration: Non-Zero Bending Initial Conditions, No Twist, Trailing Edge Sensor

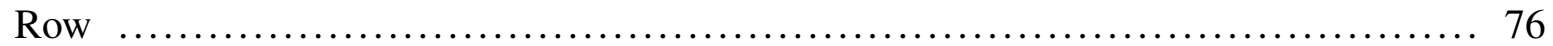

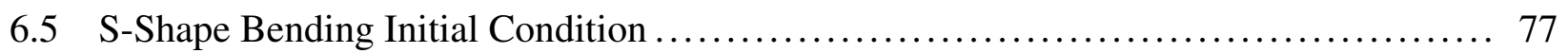

6.6 Initial Conditions: S-Shape Bending, No Twist, Leading Edge Sensor Row ........... 79

$6.7 \quad$ Initial Conditions: S-Shape Bending, No Twist, Trailing Edge Sensor Row ........... 80

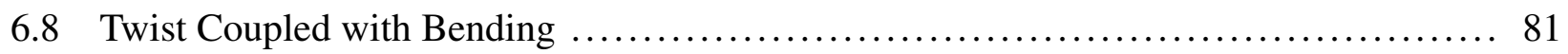

6.9 Initial Conditions: Twist Coupled with Bending, Leading Edge Sensor Row ........... 82

6.10 Initial Conditions: Twist Coupled with Bending, Trailing Edge Sensor Row .......... 83

6.11 Kalman filter performance over an extended periods of time $\ldots \ldots \ldots \ldots \ldots \ldots \ldots \ldots \ldots . \ldots 5$

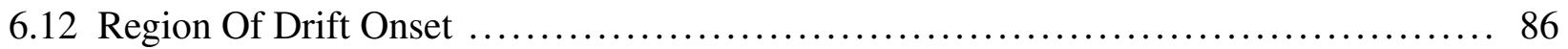

6.13 Wind Tunnel Test: 2.5 PSF, $15^{\circ}$ AOA:Leading Edge Sensor Row $\ldots \ldots \ldots \ldots \ldots \ldots \ldots .87$

6.14 Wind Tunnel Test: 2.5 PSF, $15^{\circ}$ AOA: Trailing Edge Sensor Row $\ldots \ldots \ldots \ldots \ldots \ldots \ldots$

A.1 Sensor: ARAMIS Adjustable Base 6M (Courtesy of GOM) $\ldots \ldots \ldots \ldots \ldots \ldots \ldots \ldots \ldots \ldots 98$

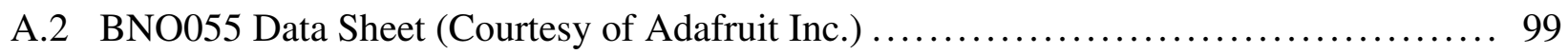

A.3 Arduino Nano Data Sheet (Courtesy of element14) $\ldots \ldots \ldots \ldots \ldots \ldots \ldots \ldots \ldots \ldots \ldots \ldots \ldots$ 


\section{LIST OF TABLES}

Table

Page

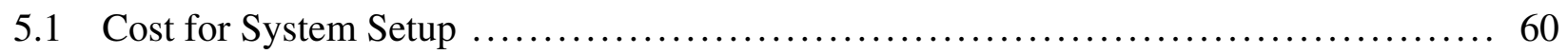

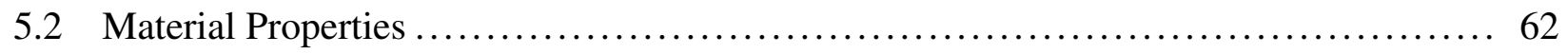

$6.1 \quad$ Mean Square Error for Measurement, Prediction and Estimate. .................... 74

$6.2 \quad$ RMS Error Table for S-Shape Initial Condition $\ldots \ldots \ldots \ldots \ldots \ldots \ldots \ldots \ldots \ldots \ldots \ldots . \ldots$

6.3 RMS Error Table for Combined Bending and Twist $\ldots \ldots \ldots \ldots \ldots \ldots \ldots \ldots \ldots \ldots \ldots . \ldots . \ldots . \ldots$

6.4 RMS Error Table for Wind Tunnel Test $\ldots \ldots \ldots \ldots \ldots \ldots \ldots \ldots \ldots \ldots \ldots \ldots \ldots \ldots \ldots \ldots$ 


\section{ACRONYMS}

\begin{tabular}{|c|c|}
\hline $\mathrm{AOI}$ & Angle of Incidence \\
\hline FEM & Finite Element Method \\
\hline $\mathrm{GPa}$ & Gigapascal \\
\hline HFDF & High Frequency Direction Finding \\
\hline MEMS & microelectronic mechanical sensors \\
\hline RMSE & Root Mean Square Error \\
\hline \multicolumn{2}{|c|}{ GREEK SYMBOLS } \\
\hline$\phi$ & bending angle \\
\hline$\phi_{i}$ & Hermite cubic interpolation function \\
\hline$\psi$ & weight function \\
\hline$\rho$ & density \\
\hline$\theta$ & twist angle \\
\hline \multicolumn{2}{|c|}{ OTHER SYMBOLS } \\
\hline$w$ & displacement in $\mathrm{z}$ direction \\
\hline $\mathcal{D}$ & flexural rigidity \\
\hline$c$ & chord length in meters \\
\hline$c_{i}$ & state vector \\
\hline$h$ & plate thickness in meter \\
\hline$L$ & lift force \\
\hline$l$ & wing span in meters \\
\hline$M_{x}$ & approximation function \\
\hline$R$ & error residual \\
\hline
\end{tabular}




\section{CHAPTER 1 \\ INTRODUCTION}

Much of an aircraft's flight condition and configuration is captured by means of advanced sensor technology. Airspeed is obtained using high accuracy static and dynamic pressure probes; altitude can be determined by means of precision barometers and air data computers. In modern aviation, a commercial aircraft's data acquisition system delivers a wide array of useful information regarding the flight and the aircraft's operational state in real time. Even though knowledge of a wing's physical shape can be most pertinent to the design of adaptive and morphing wing controls or structural health monitoring, currently only limited real time structural information is available to the pilot and the flight control system. While some sensors detect the proper actuation of vital aircraft components such as the extension or retraction of flaps, spoilers and landing gear, the acquisition of structural deformation data within non-actuated components remains a challenge. In fact, when considering the structural integrity, much of the aircraft's health monitoring is conducted on the ground as part of routine maintenance intervals. Knowledge of structural deformation such as bending and torsion can be of significance to the pilot during a sudden turbulence encounter. In order for a pilot or flight control system to draw a conclusion about the aircraft's structural health, it is imperative for the real time deflection and torsion data to be measurable. With this information the pilot or control logic can then determine whether or not evasive maneuvers should be performed to return the aircraft into a state of acceptable structural stress or strain margins. Additionally, inferring the shape of a moving aircraft component or structure in flight without the use of optical interrogation techniques and extensive experimental setup has historically been a more challenging undertaking than the task itself might suggest. An ever-changing reference frame as well as the technical and logistical difficulties of incorporating a self-contained sensory system, capable of supplying sufficient information pose challenges which an engineer has to overcome in order to provide both pilot and controller logic with the required feedback.

Some methods of producing real time deflection and torsion data as well as the respective 
limitations of such methods are discussed in the next chapter. While much interest is being directed toward the proliferating research field of evolving morphing wing control and design, there is a need to advance research efforts towards the development for adequate sensing methods of shape changes and structural deformations. This research project examines methods to obtain reliable, real-time, static and dynamic bending and torsion data used to model the deformation of an aircraft component. In particular, the principal focus of the herein discussed work is the conceptual development and experimental validation of a system capable of sensing the deformation of an aerodynamic structure such as an aircraft wing in real time. For this, various sensing and processing methods were considered and evaluated for further investigation, some of which were also discussed by authors of previous work. The approach of using MEMS inertial sensors in combination with a Kalman filter design as seen in other applications offered the most promising qualities such as cost efficiency, size as well as computational performance, and was therefore favored for further exploration. Simulations of the Kalman filter logic incorporating the anticipated noise parameters for input and process noise served as a basic proof of concept. Preliminary experiments of a kinematic approach for flat plate under static and dynamic loads further validated the concept but also identified limitations of such an approach. Those limitations were addressed in an extension to the kinematic approach by introducing a finite element model. This structural dynamic model was used to predict the deflection of the flat plate structure. The data was mapped to the sensed deflection output data in order to obtain a state estimate, used to synthesize the displacement at any location between sensors. This method enhanced the robustness and accuracy of the system.

\section{$1.1 \quad$ Literature Review}

Environmental, weight reduction, and performance considerations have steered modern aircraft research and development in a direction where an airplane will be able to dynamically change its characteristics and configuration, morphing and adapting to various flight conditions and performance envelopes [1, 2]. Present morphing wing research essentially incorporates three categories which investigate the control, the actuation and the sensing of the morphing structure. 
Variable airfoil geometry control was investigated for the purpose of drag reduction. The main objective of the referenced work by Popov et al [3-5] was to develop a system for the active control of wing airfoil geometry during flight to allow drag reduction. Simulations and experimental work were used to successfully validate results. Extensive work has been done on the subject of morphing wing control and actuation: Menon et al [6] are investigating the flight dynamics model of an aeroelastic aircraft wing structure. Preceding this work was extensive research on the control and drag reduction of morphing wings. Particular focus was directed at the actuation of variable trailing edge [7-[11]. flap control system. Their objective is to implement an adaptive control design that will change the wing shape, more specifically the trailing edge and consequently maximizing lift in various flight regimes. The implementation of smart material actuators to include shape memory alloys (SMA), piezoelectric actuators (PZT), and shape memory polymers (SMP), is investigated by Sofla et al. [12]. A similar approach was pursued in work conducted by Vos et al. wherein a new type of piezoelectric actuator was used to achieve the desired trailing edge deflections [13].

Knowledge of aircraft shape and structure, particularly wing deformation is not only useful as an input for a control design but also structural health monitoring. Generally speaking, there are two most commonly used methods for quantifying structural deformation, which can be classified as strain methods and optical interrogation techniques, both of which have been investigated thoroughly. Van der Draai et al.[14] have investigated two techniques, namely the Painted Pattern Method and the Projected Pattern method whereby detected distortions in the pattern that extend across- the wing surface are compared to actual measurements along sections of the wing using a 3D coordinate measuring device and theoretical predictions. Similarly, Veerman et al [15] describe measuring actual wing deflection under static and dynamic loading by a technique referred to as Image Pattern Correlation Technique (IPCT). This also non-invasive method correlates the change of closely spaced dot patterns to a deformation of a wing structure under various structural loadings [15]. Davis [16] discussed the use of videogrammetry wherein a camera, mounted onto the tail of the aircraft tracks various targets across the wing structure. 
Experimental wind tunnel testing yielded acceptable results; however, the author recommends optimizing camera placement and more advanced imagery equipment. Research efforts of Frost et al [2] present an extension to such classical optical methods with a central focus on improving the overall performance of conventional machine vision systems. Their findings describe the development of a biomimetic optical sensor to improve the performance of conventional sensor in terms of weight, power consumption and computational requirements. On a smaller scale Dixon et al [17] have examined a fiber optic interferometry approach on the basis of amplifying small transverse deflections. The results suggest a high accuracy; however, replicating this method for larger scale deflections would likely not be feasible and the required aperture difficult to implement. As part of NASA's HIMAT flight program an advanced technique for measuring wing deflection was developed using an array of infrared LEDs and sensors [18, 19].

Extensive research has been directed towards methods correlating deformations to more easily quantifiable strain measurements. A thoroughly investigated approach that is often implemented in structural health monitoring and can easily be related to deformations is the fiber optic Bragg grate method whereby the modal behavior of optical fibers within structures is analyzed and related to strain and displacement [20, 21]. Deriving displacement information from strain data as received from strain gages along a structure is also discussed in work done by Bogert et al. [22].

In recent years a new approach of using SansEC smart sensors has been investigated. A sensor patch is adhered externally to a structure that is statically and dynamically deformed. A deformation such as bending or torsion will change the frequency response of the patch relating a frequency shift to the change of the geometric shape [23, 24]. While this method shows advantages such as fault and damage tolerance in conjunction with simple manufacturing and implementation, experimental results also suggest an undesirable error distribution and volatility in accuracy and reliability. This can mostly be attributed to its sensitivity to external factors such as electro and ferro magnetic interferences as well as changes within the immediate operating environment such as atmospheric fluctuations i.e. temperature, humidity etc., which can significantly skew the sensor 
output unless such parameters are properly accounted for.

A vastly different approach to obtaining the desired deformation measurement would conceivably be the correlation of distance to signal attenuation as discussed by Luo et al [25]: An RFID tag system was used inversely to infer the exact position of the tag reader. This method however shows inadequate resolution to be adapted for further development to accurately measure structural deformation. Similarly using a radar system as described by Bahl and Padmanabhan [25] would yield comparable drawbacks.

A technique of estimating the unknown aero-elastic state vectors was described by Nhan et al. whereby constructing a state observer using accelerometers provides a good example of supplying estimated states to a morphing wing control design as is investigated herein [1]. The concept of using inertial sensors such as accelerometers and gyros for the purpose of sensing deflection has become an appealing approach in part due to the technological advancements in size, performance and availability of modern sensors.

Research was conducted by Gong et al [26] wherein the authors describe experimental validation of mathematical models for both accelerometers and gyroscopes. Obtained estimates were compared to data obtained from a conventional large form factor IMU (Inertial Measurement Unit). The author's conclusion suggests desirable performance characteristics of accelerometers and gyros, however the dimensions of IMUs used in the experiment would likely be impractical for the implementation on an aircraft structure. Of particular interest is the work by Kowalczuk and Merta who investigated position and displacement of a Robot using MEMS accelerometer and gyro sensors in conjunction with a Kalman filter to reduce the integration error buildup [[27]. Experimental results show desired performance in that the system is robust and can estimate the position of a robot within acceptable error margins. Work done by Shark et al. illustrates the usage of a Kalman filter in conjunction with an accelerometer in order to estimate the time history of a boat's vertical displacement while floating when exposed to wave perturbations [28]. Their findings show that the applied Kalman filter is successful in estimating small displacements and capable of preventing the rapid error accumulation due to the cyclic double integration of the 
accelerometer data, thus providing sound reason a similar configuration can be used on an aircraft wing structure.

\subsection{Contribution}

The previously discussed work presented by other researchers provides a basis for the adaptation of described methods and the development of new approaches towards the goal of measuring structural aircraft deformation. It is important to mention that while many of the described approaches yield adequate results, implementing and possibly integrating these methods in the manufacturing process poses difficulties and in certain cases proves unfeasible. For instance, experiments conducted with conventional visual interrogation methods or fiber optic interferometry require extensive setup and calibration. While acceptable within the developmental and engineering flight test environment, such methods would be unsuitable for the permanent installation on an aircraft. The primary contribution of the work described herein is:

1. The analysis and evaluation of new approaches and concepts

2. The expansion from previous research choosing feasible sensing methods

3. The design, implementation and finally experimental testing, including wind tunnel testing of static and dynamic structures in order to validate the sensor system on an actual wing.

A starting point for this project was the notion of surveying an aircraft wing by using electromagnetic ranging. Conceptually analogous to radar, this novel approach would capture the distance and displacement of pre-defined grid points across the wing surface by relating the distance to signal attenuation and the time it takes the signal to travel between transmitter and receiving grid point. Basic experiments using a signal generator, oscilloscope and network analyzer identified the capabilities and limitations of this approach. The latter of which inspired a new process, in which the displacement between transmitter and receiver was correlated to a shift in phase or amplitude. For the acquisition of torsion data, initial experiments were conducted using a polarized high frequency RF signal. The signal strength at the receiving dipole antenna was examined as it was undergoing a coplanar rotation with reference to the transmitting dipole 
antenna to determine if the corresponding signal attenuation can be sufficiently related to twist of a structural component.

The previously summarized experiments have helped to identify the inherent limitations of the associated methods. A more robust and reliable approach of using MEMS sensors was favored and refined by introducing a Kalman filter state estimator which filters and fuses sensor information from multiple sensors to obtain the most probable deflection or torsion estimate. Investigative efforts discussed by other authors have not been applied to any aircraft component or utilized for the purpose of sensing structural deformation. Most methods described by their work are limited to a single point of interest to which a Kalman state estimator is applied. To expand on this concept, the components of this project are 1.) the implementation of a structural dynamic model in order to observe the dynamic behavior of the wing when exposed to actual flight conditions. 2.) The development of a state observer which estimates the wing deformation at various points required to compute the response of the wing in real time. The following Chapter 2 will revisit the problem statement and discuss various approaches which were considered and investigated. Chapter 3 will provide a detailed account of the method selected for preliminary testing and discuss the experimental setup and testing prcocess. Preliminary results for the kinematic approach are discussed in Chapter 4. Chapter 5 will expand on the findings by addressing the limitations of the kinematic approach in replacing the kinematic equations of motion with a finite element model. Results for the finite element method will be shown and discussed in Chapter 6 , The final Chapter 7 will reflect on the methods and results obtained and discuss future work. 


\section{CHAPTER 2}

\section{METHOD OVERVIEW}

For this project previous research was examined to determine which methods are presently or could potentially be used to detect structural changes. Several concepts could conceivably be expanded on to achieve the desired measurement of structural deformation. Much emphasis was

directed at exploring novel approaches in order to solve the central problem of sensing the shape of an airplane wing. The various methods that were investigated are 1.) Interferometry Approach, 2.) Pulse Width Modulation, 3.) described within this section, 4.) Polarized signal attenuation 5.) SansEC self-resonating sensor patches, and 6.) Inertial Sensors.

\subsection{RF ranging and signal processing}

Using high frequency radio signals for electromagnetic ranging in order to obtain position and consequently displacement information hypothetically provided a conceptually sound and noninvasive approach [30]. In early stages of this investigation a central concern was to determine which type of medium would be most capable of acquiring the small changes in position over time.

At first, tests were conducted on the propagation of high-frequency waves. For this, a series of simple experiments were set up in order to explore both the capabilities and the limitations associated with the various methods and to confirm findings expressed in other research. These included two interferometry approaches which explore the effect of displacement between transmitter and receiver on changes in amplitude and phase. For the purpose of detecting torsion, rotational displacement was examined by evaluating polarized RF signals. Next, the use of SansEC self-resonating patches was considered and discussed. Finally, the use of inertial sensors in combination with a Kalman filter state estimator was investigated and favored for further development and implementation. 


\subsubsection{Interferometry Approach (Amplitude)}

Among the first theories to be explored was a concept based on interferometry. High frequency signals of known amplitude were considered to be transmitted omni-directionally from the wing root. The signals which propagate from two separate transmitting sources S1 and S2 generate interference by overlapping as seen in Fig. 2.1. The resulting wave is measured at Sf which is the arithmetic sum of the two waves S1 and S2.

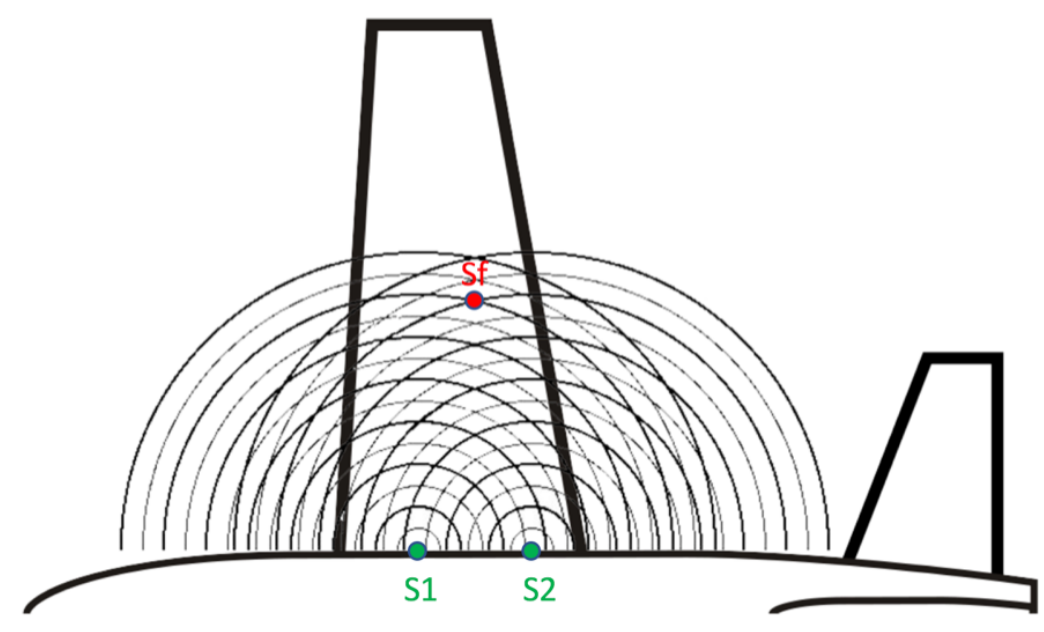

Figure 2.1: Interference Pattern

Depending on the phase angle as determined by the distance traveled to the receiving antenna from either transmitting antenna, these waves will form intermediate peak values between the interference minimum and maximum as shown in Figure 2.2a and 2.2a

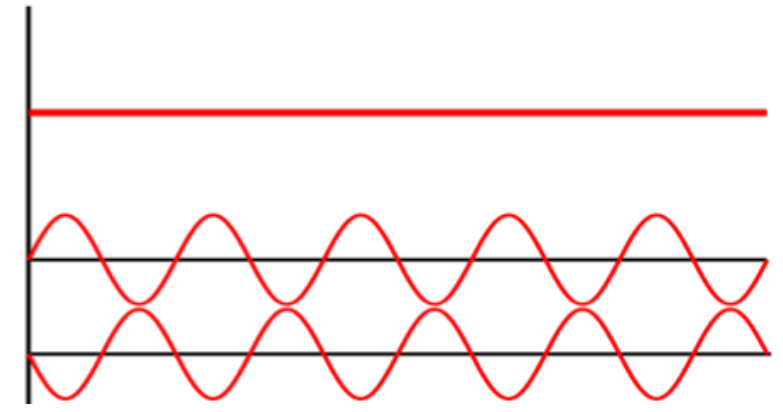

(a) Interference Minimum

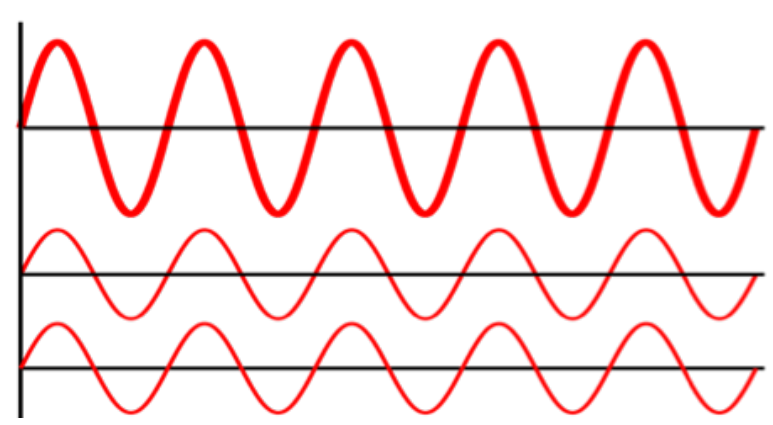

(b) Interference Maximum

Figure 2.2: Signal Interference 
Eq. 2.1 and Eq. 2.2 show the relationship between the two signals and the resulting interference as a function of space and time

$$
\begin{aligned}
S_{1}+S_{2} & =S_{f} \\
S_{f}(x, t) & =A \sin (k x-\omega t)+A \sin (k x-\omega t+\phi) \\
& =2 A \cos \left(\frac{\phi}{2}\right) \sin \left(k x-\omega t+\frac{\phi}{2}\right)
\end{aligned}
$$

Using interference patterns as shown in Fig. 2.1, the location of a sensor can be inferred by relating changes in amplitude to position relative to interference maximums and minimums. An experimental setup in the WSU General Aviation Flight Lab served as a proof of concept using a high frequency RF signal of approximately $2 \mathrm{GHz}$. The signal was separated into two synchronous signals using a splitter to be transmitted from antennas S1 and S2 which were approximately one meter apart. A receiving antenna Sf, which was connected to an oscilloscope was used to probe and identify interference maxima and minima in order to relate the displacement of the receiving antenna to measurable changes in magnitude. This method, if found effective, would provide displacement information of individual grid points used to assemble a structural model of the wing to later on include both deflection and torsion. However, it was found that interference from external sources created a significant amount of noise and skewed the measured amplitude

\subsubsection{Pulse width modulation using a signal multiplier}

For this method the time-of-flight of an electromagnetic impulse was considered. The approach is based on the premise that the deformation of a structure such as bending or torsion results in a change of mechanical quantities including the distance between a reference point and a measurement point. A distance change can conceivably be quantified by examining the time-offlight between the points with that of a reference signal. The theory behind this concept is based on modulating the pulse width of a square wave signal by multiplying a received signal by the transmitted reference signal. The phase difference between the impulse waves and the resulting pulse width of the product wave can then be related to the distance between the corresponding 
transmitter and receiver.

First, it is assumed that impulse waves of equal frequency can be maintained at the point of measurement and point of reference. A comparison of the transmitted, unaltered signal at the reference point and the measurement point is conducted. With an increase in distance between reference and measurement point, the signal will arrive with delay. The opposite is true whenever the distance is reduced. The mentioned time delay is expressed as a phase shift between measured and reference signal. The phase difference between two impulses can be described by the duty cycle, an expression of time during which, at a measurement point, the non-zero states of both impulses coincide. Figure 2.3 illustrates the concept of applying pulse width modulation to the given problem statement.

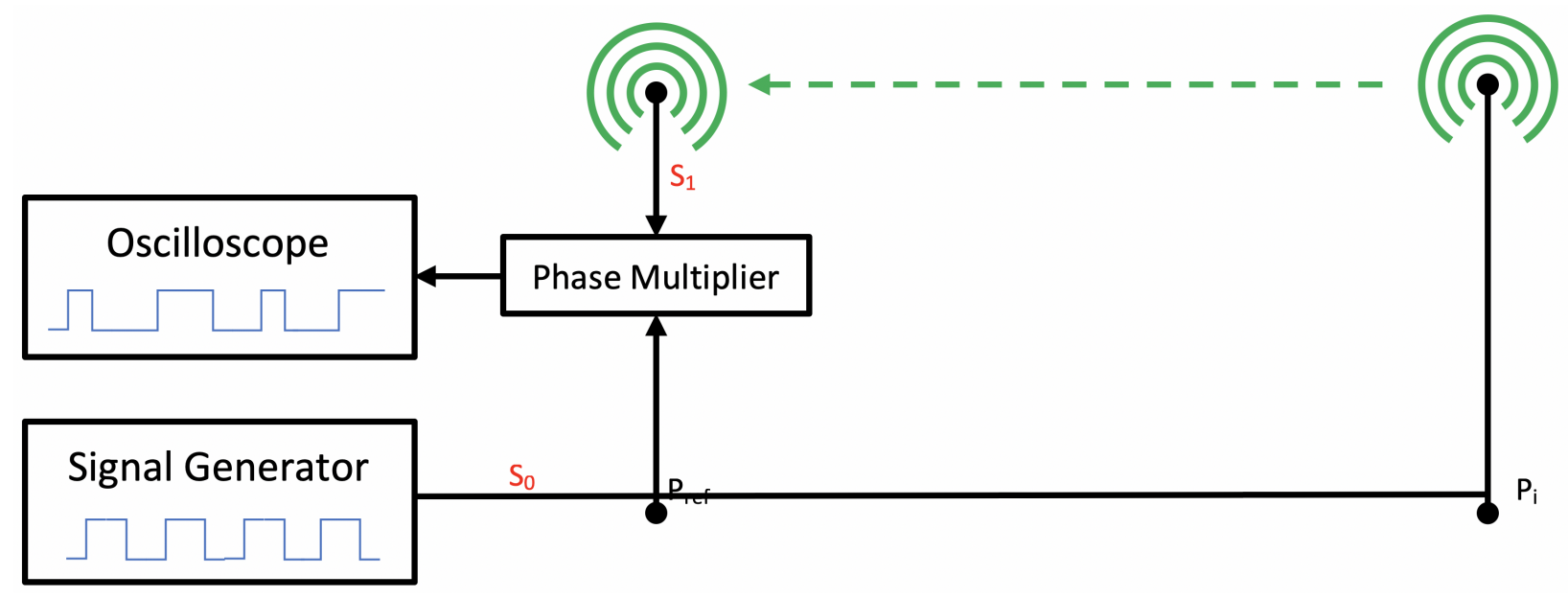

Figure 2.3: Conceptual graphic of pulse width modulation

A high frequency square wave signal is routed such that it originates at a reference point $P_{\text {ref }}$ (likely located near the wing root) from there travels via hard-wire to a point of inspection $P_{i}$. There, it will wirelessly return to $P_{r e f}$. Upon arrival, the signal $S_{1}$ will be multiplied with the outbound signal at the origin $S_{0}$. When multiplying two impulse waves of magnitude 1 , the resulting wave form will also be a square wave of magnitude 1. An appropriate equivalent electronic circuit to implement the multiplication is a logical operator to evaluate the duty cycle also called an AND-gate. Table 1 shows the switching logic of such a gate. The AND-gate sets the potential to 1 only during intervals where both, input S1 and S2 carry a non-zero potential 
simultaneously. Figure 2.4 and Figure 2.5 illustrate the phase shift and corresponding duty cycle of two impulse sequences with identical frequency but varying displacement of $1 / 3$ pulse width and $2 / 3$ pulse width respectively.

A (S1)

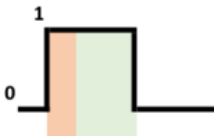

B (S2)

A $\times$ B
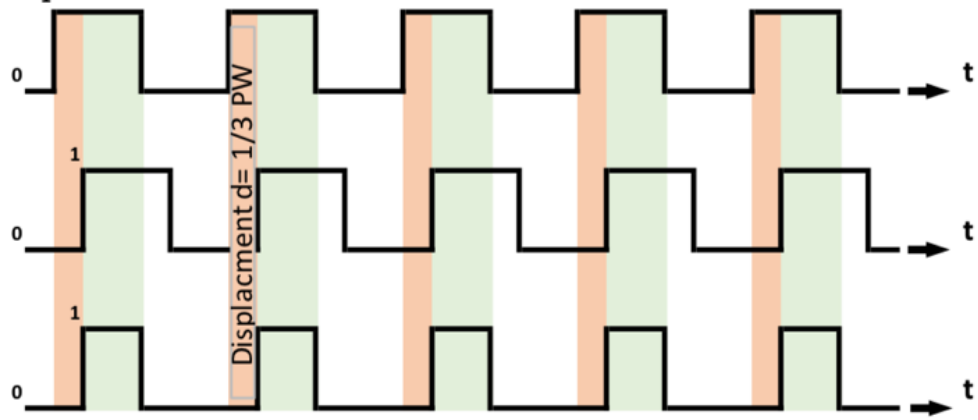

Figure 2.4: Impulse arrives with a delay of $1 / 3$ pulse width

A (S1)

B (S2)

0
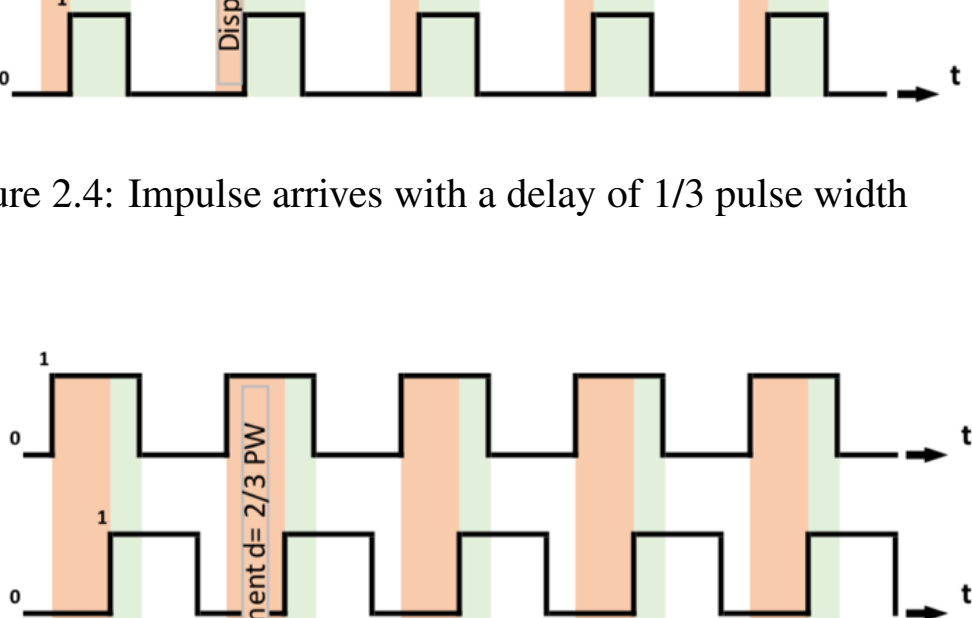

n

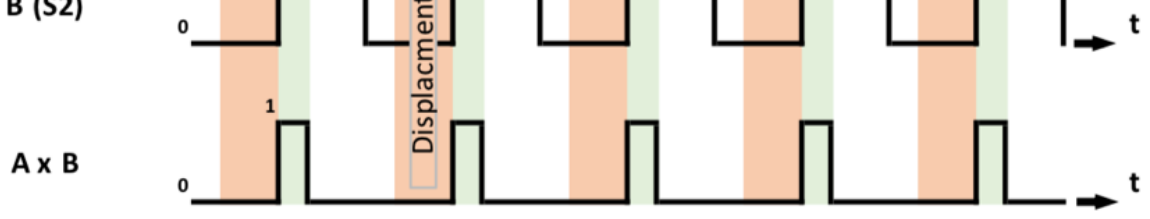

Figure 2.5: Impulse arrives with a delay of $2 / 3$ pulse width

The described method has conceptual drawbacks, however, which render this approach less desirable. First, the technique allows for detecting only a small range of displacements of exactly one half the pulse width. It cannot be assumed that the structural deformation can be limited so as to exclusively fall within this range. Also, in order to transmit a signal between two points, the antenna properties of the receiver and transmitter must match the corresponding frequency. Based on the structural dimensions of an aircraft this poses a controlling limit to the maximum wavelength that can be used in order to accommodate several antennas in the wing and fuselage structure. For example, a wavelength of 5 meters, which would presumably provide adequate resolution and 
assure most deflections to be captured, requires a 2.5-meter antenna dipole equation (2.4) and a resulting frequency of $600 \mathrm{Mhz}$ equation (2.5)].

$$
\begin{aligned}
& l_{A}=\lambda / 2=5 \mathrm{~m} / 2=2.5 \mathrm{~m} \\
& f=\frac{c}{\lambda}=\frac{3 \times 10^{6} \mathrm{~m} / \mathrm{s}}{5 \mathrm{~m}}=600 \mathrm{Mhz}
\end{aligned}
$$

Even for a typical Boeing 737-800, which has a wing span of 36 meters it would be difficult to install an array of antennas with such dimensions. A reasonable frequency range is therefore assumed to fall between $1 \mathrm{GHz}$ to $2 \mathrm{GHz}$. However, for this frequency range signal generators cannot synthesize a true square wave. With an increase in frequency, the steep sides flatten out. This behavior then makes a proportional evaluation no longer possible. It was therefore decided to direct research efforts towards other more favorable directions.

\subsubsection{Polarized signal attenuation approach}

There are several ways to utilize an antenna in order to determine the direction or angle of incidence from which a captured signal originates. For this, antenna types and design were considered to explore a potential adaptation to allow short distance directional measurements. The considered implementation would be comprised of a transmitting dipole at the wing root, perpendicular to the wing chord and multiple receiving dipoles parallel to the wing chord as shown in Figure 2.6. Since the early days of radio technology the loop antenna has been a well-known antenna type used to track the angle of incidence (AOI) of a received signal for various purposes such as navigation, tracking and ranging of signals [29]. The advantage of a loop antenna is its ability to receive both vertically and horizontally polarized signals. For the purpose of this research an experiment was conducted: a signal was polarized in one direction: both transmitting and receiving antenna were horizontally aligned. Therefore, a simplified antenna design was selected, consisting of a half-wave dipole with length of $l=\lambda / 2$ Figure 2.6. The experiment was carried out with a "stretched" dipole for the transmitter, and a folded dipole for the receiver. 


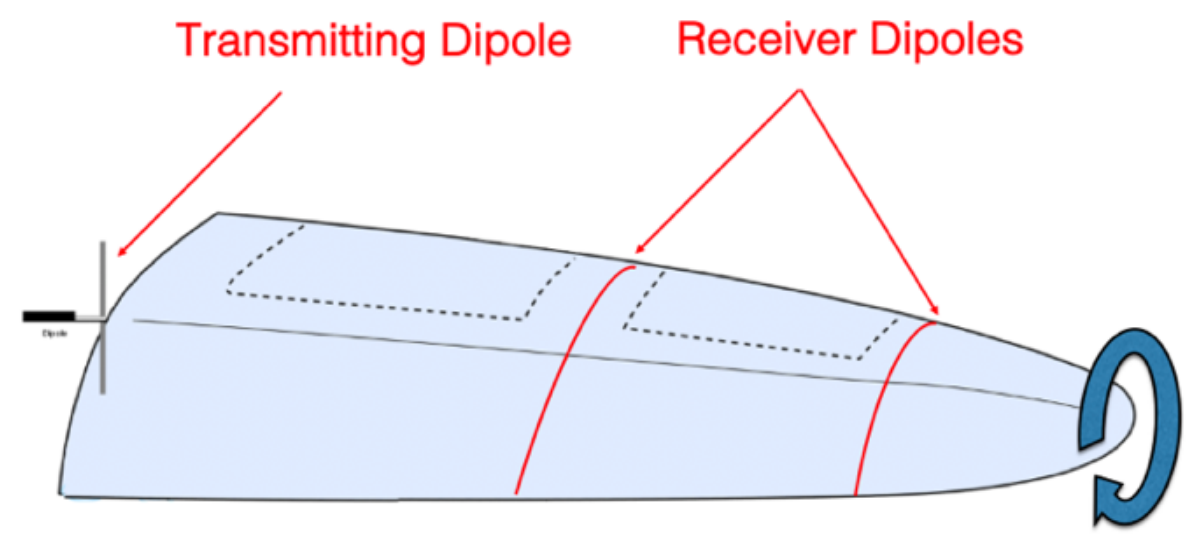

Figure 2.6: Conceptual Dipole Installation

Based on the relation $f \dot{\lambda}=c$ and a selected frequency of $100 \mathrm{MHz}$, the corresponding wavelength resulted in 3 meters. The material dependent length correction which accounts for the conductor diameter was neglected as it would only minimally influence the results. Figure 2.7 shows the theoretical ideal gain or attenuation characteristic of a horizontally aligned dipole.

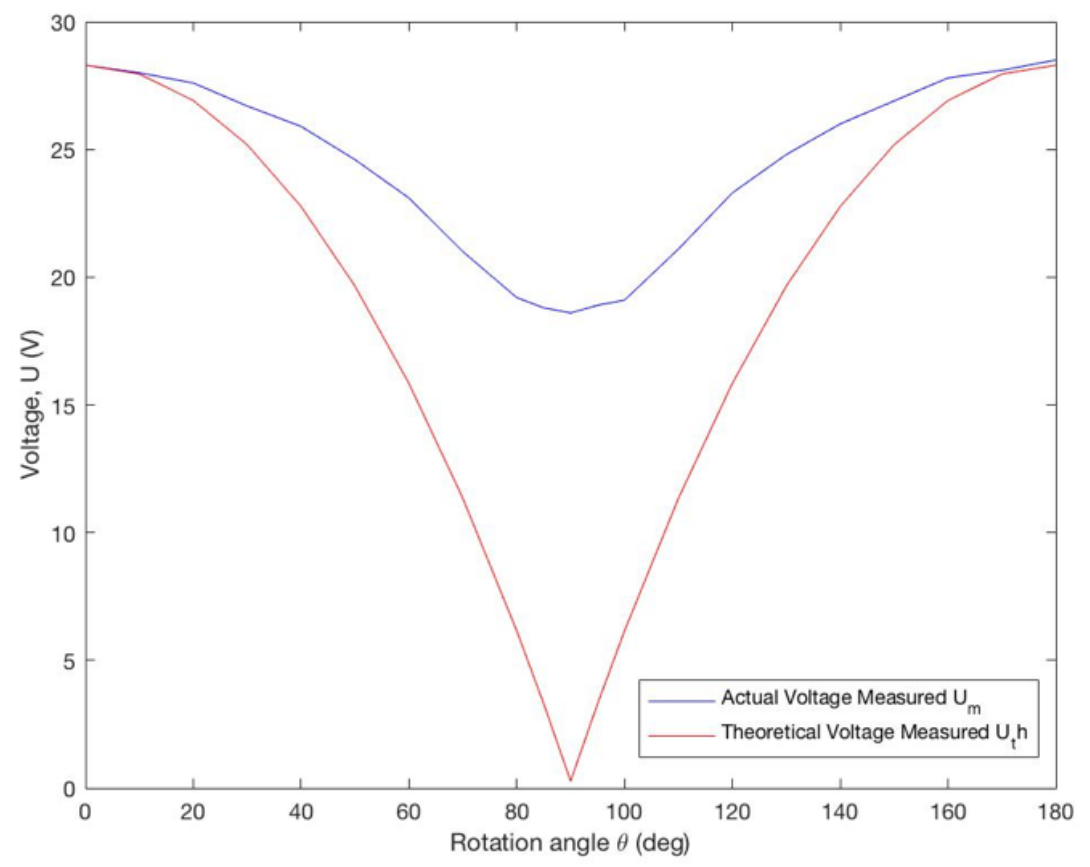

Figure 2.7: Dipole directional signal attenuation 
It illustrates the co-planar, directional signal attenuation in terms of gain with respect to orientation offset angle between the transmitting and receiving antenna. Of particular interest are the extreme minimum signal gain and maximum attenuation regions, near $90 \circ$ and $270 \circ$ of angular offset where the greatest directional sensitivity in $\Delta d B / \circ \mathrm{rad}$ is evident. Historically, high frequency direction finding (HFDF) has therefore been a technique of tracking the minimum gain.

It is reasonable to assume the signal attenuation of a polarized signal is directly related to the direction at which it enters the receiving antenna as shown in Figure 2.7. The described principle was adapted in an experimental arrangement wherein a receiving dipole was gradually rotated with respect to the fixed transmitting dipole in increments of $10^{\circ}$ angular offset. Figure 2.8a and Figure 2.8b shows the experimental setup of receiving and transmitting dipole respectively.

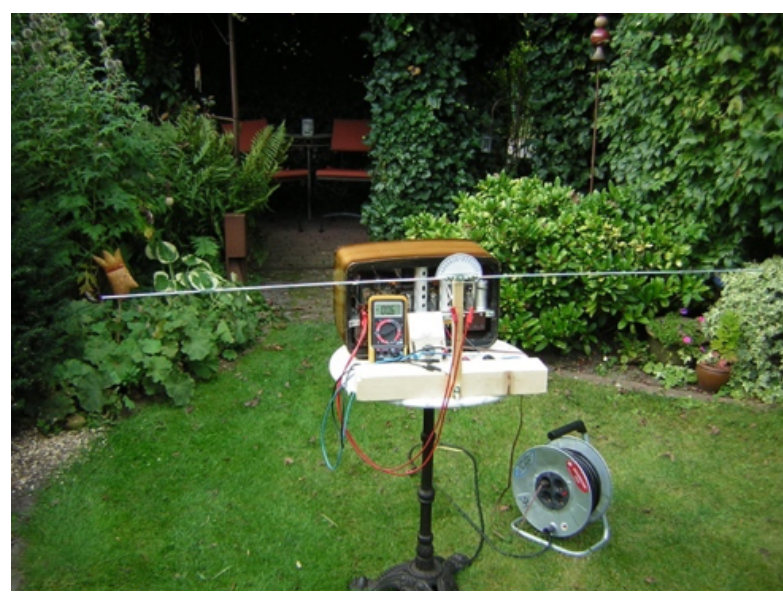

(a) Dipole Receiver

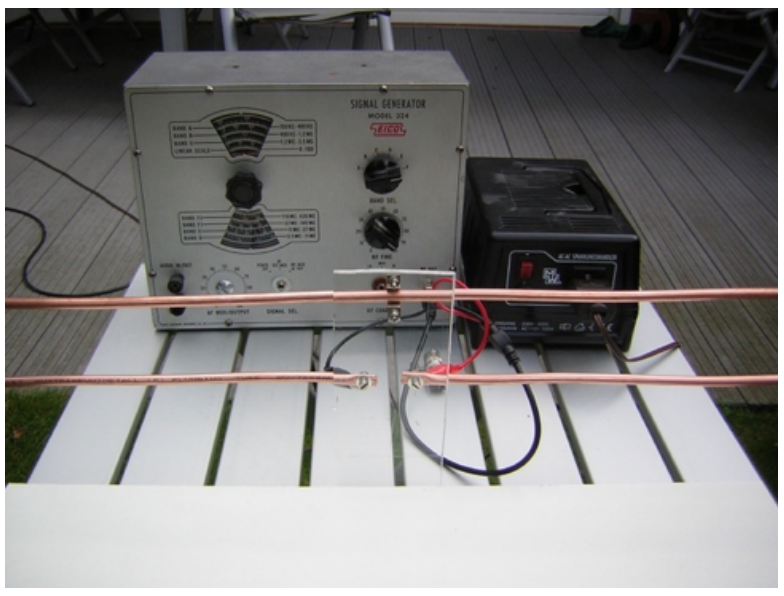

(b) Dipole Transmitter

Figure 2.8: Experimental Dipole Setup

The receiver voltage was measured as a dependent quantity of the angle of incidence and compared to the receiver voltage at an AOI of zero degrees. The observed results of the experiment, however, varied vastly from the theoretical attenuation curve depicted in Figure 2.7 Testing revealed that the observed maximum attenuation near the region of $90^{\circ}$ or $270^{\circ}$ angular offset is not as clearly defined as the theoretical plot suggests. The depicted plot shows a more shallow and diffuse signal loss rather than an anticipated narrow peak. This can likely be 
attributed to two factors: First, the antenna arrangement was set up within finite height above ground with non-negligible capacitance and secondly the diffuse reflection off of objects within the immediate surroundings. The experiment is conclusive in that it renders any method relying on the relationship between amplitude changes to either bending or twisting as deficient. It was therefore decided to discontinue any further investigation of the method and direct efforts towards a more favorable approach.

The alternative to analyzing the dependence of deformation to changes in signal strength was at least the theoretically conceivable notion of relating a phase shift to the distance between transmitter and receiver. However, this approach was excluded from further investigation due to the anticipated diffuse reflection from the surrounding environment and the associated interference. Furthermore, in order to sufficiently evaluate small changes in displacement an ultra-high frequency signal and associated short wavelength would be required to adequately measure changes in pulse width and to relate those to potential bending or torsion.

\subsection{SansEC Sensor Patch Approach}

A unique approach to quantifying wing deformation in real time with the use of a smart sensor was investigated. The SansEC sensor which was explored for this application was first developed and introduced by NASA as a universal sensor type, capable of performing measurements to detect multilateral physical properties within its surroundings [30]. The sensor resembles a self-resonating coil that is etched or printed onto an adhesive sheet. The notion of this method suggests that deformation of an aircraft wing due to aerodynamic loading or morphing will change the frequency response of the sensor. The sensor itself is excited by electromagnetic waves from an interrogating antenna and evaluated using a network analyzer. When the physical shape of the patch changes, its self-inductive characteristics are altered. The resulting change in frequency response is detected by the network analyzer and can be related to the deformation. Figure 2.9 shows a close-up image of a sample SansEC patch which can be manufactured to match various patterns and sizes. 


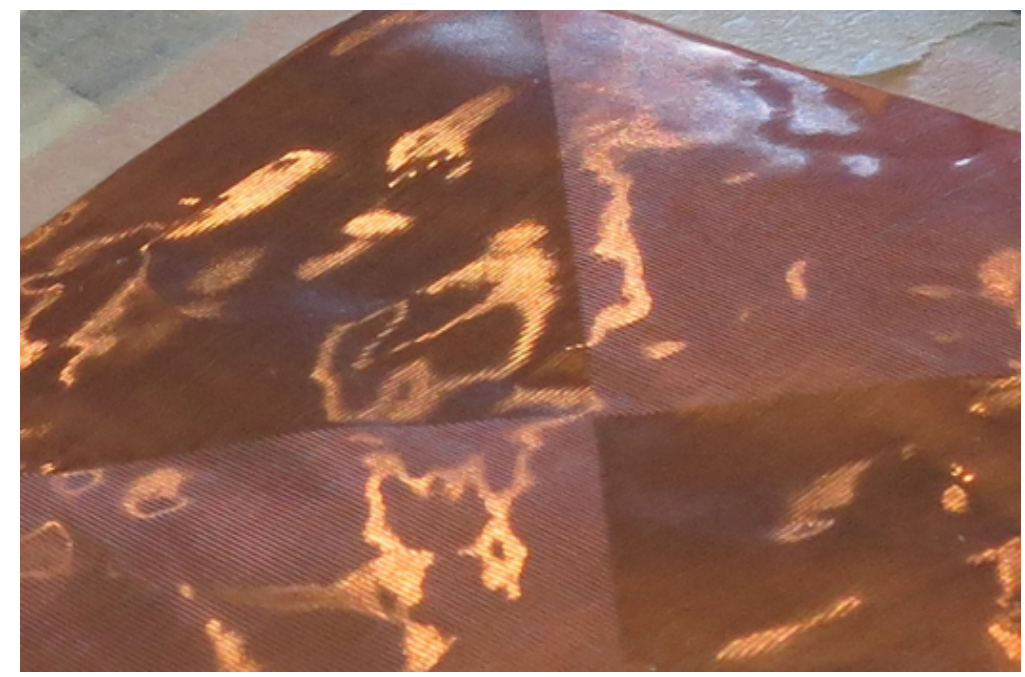

Figure 2.9: SansEC smart sensor patch

An experimental arrangement for this method consisted of a foam model aircraft with a flexible wing structure, two SansEC sensor patches, installed respectively on each wing of the model and a graphite electrode for each patch installed at close proximity as shown in Figure 2.10.

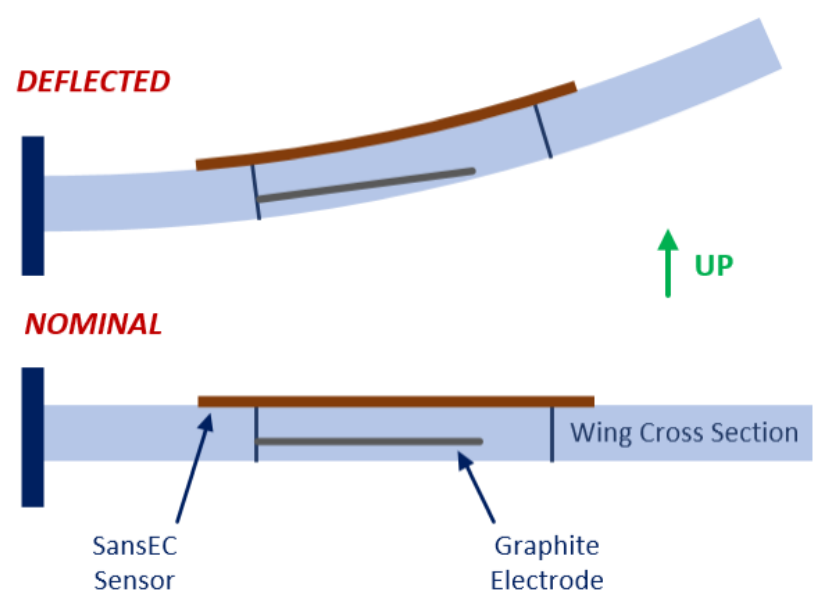

Figure 2.10: Conceptual SansEC installation

The graphite electrode enhances the change in frequency response by penetrating and attenuating the electromagnetic field measured by the antenna, thereby amplifying the measurable effect of the deformation. Wind tunnel tests with varying angle of attack and side slip angle were conducted to show sufficient evidence that such the described approach holds viable results when 
used on an airplane wing. Figure 2.11 shows the model aircraft subjected to actual flight conditions in the Walter Beech Wind Tunnel.

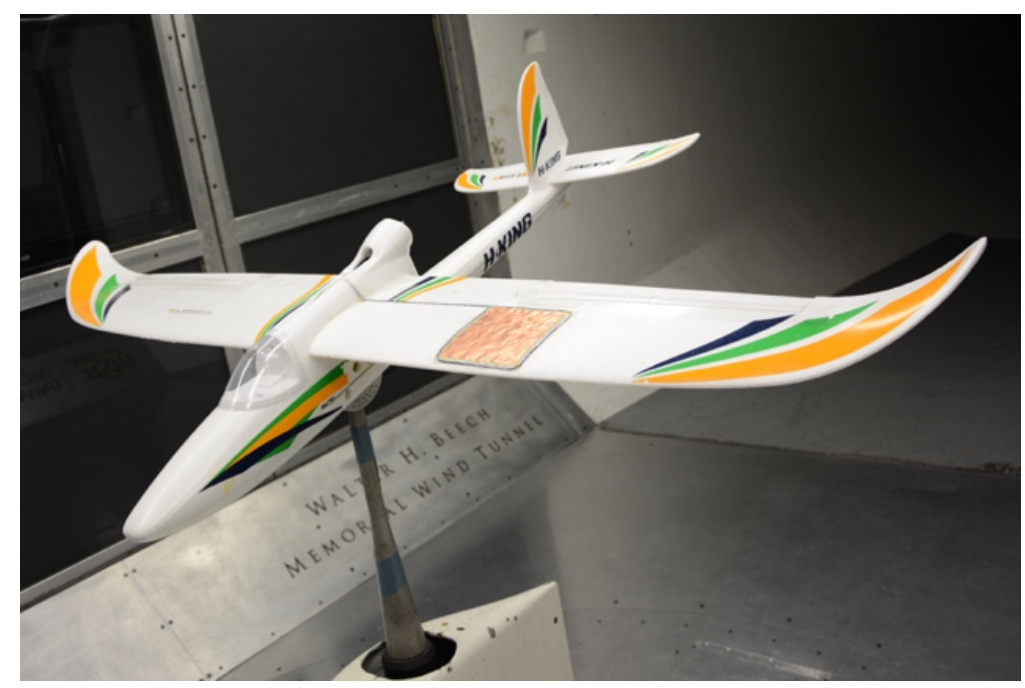

Figure 2.11: Sansec wind tunnel setup and testing

Figure 2.12 shows the relationship between the wing deflection and frequency response the sensor patch at varying angles of attack. Deflections depicted in the figure were obtained using multiple cameras, positioned such that an adequate approximation of the wing structure could be made.

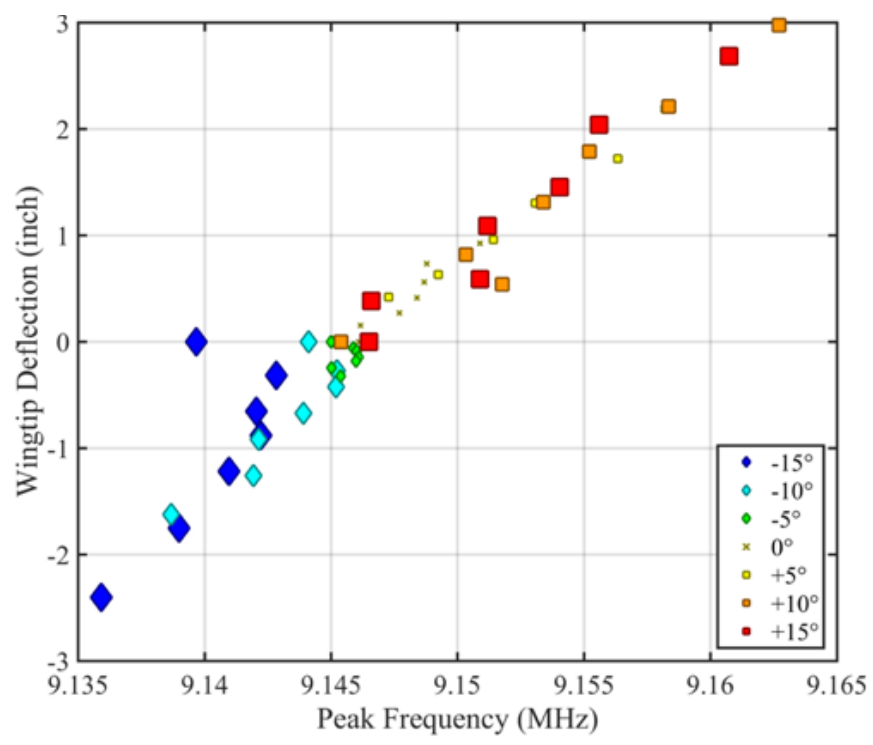

Figure 2.12: Frequency shift as result of deflection 
The described approach identified the potential of using such technology as a foundation for sensor system to monitoring aircraft wing deflection. At this stage of the development it was found to be vulnerable to other unwanted external influences such as temperature, a ferro-magnetic environment and electromagnetic interference, all of which skew the results and would require additional filtering. The method of using a SansEC patch to quantify wing deflection is currently still being investigated by other research groups at WSU.

\subsection{Inertial Sensor Analysis Methods}

Based on the limitations of previous approaches which were revealed in both analysis and experiments, an alternate method to utilizing electromagnetic signals was considered. The use of inertial sensors was explored as such sensors are capable of detecting translational and rotational motion. The obtained information can be related to the displacement of sensors. Consequently, a multitude of sensors can make up a structure and the respective motion of each sensor combined can model the deformation of such structure.

With the introduction of MEMS technology, inertial sensors have been significantly reduced in cost and size. Consequently such sensors have become more readily available and modern consumer electronics manufacturers have fostered the development of smaller and more cost-effective devices. As a starting point the focus was directed at implementing MEMS accelerometers to obtain displacement information as shown in Figure 2.13. One method of utilizing information obtained from the MEMS sensors is to take the kinematic approach: Integrating the obtained acceleration twice with respect to time naturally yields a displacement, however only relative to the lapsed time since the previous time step. Hence two valid concerns would have to be addressed: how could a reference or initial condition obtained and how would the resulting the error wind up be addressed, which was also discussed by Kowalczuk et al. [27]. 


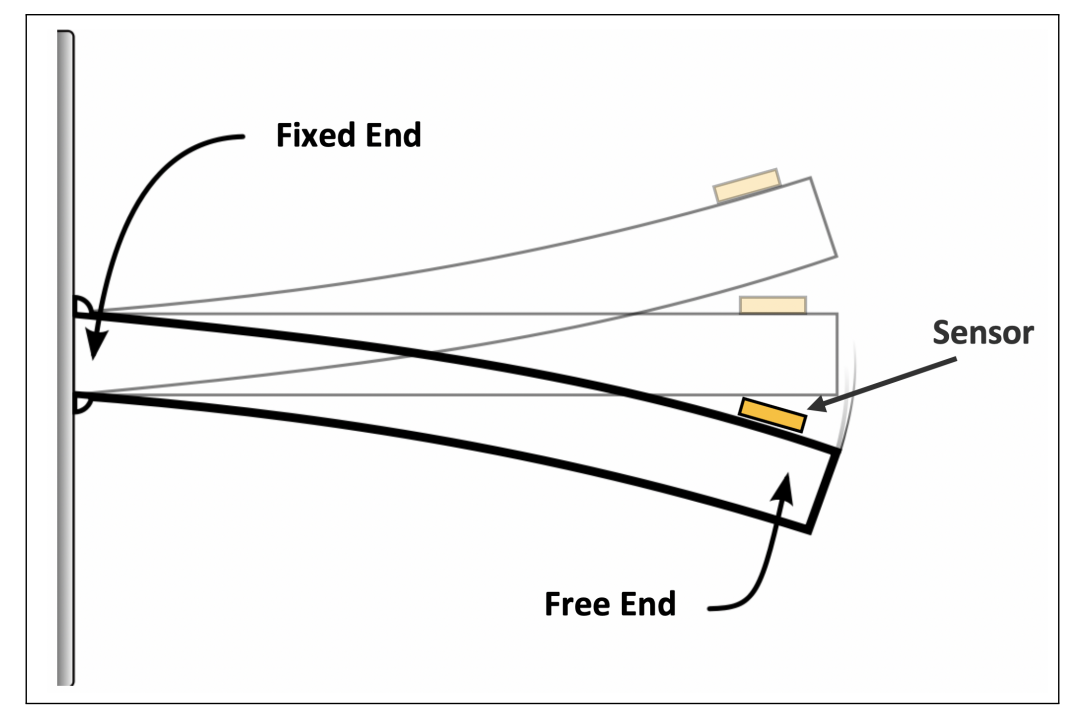

Figure 2.13: MEMS sensor application

It was therefore apparent that a mechanism would have to be introduced, capable of routinely updating an approximate position while compensating for measurement errors, which would otherwise accumulate quickly over time in the integration process. While a low pass filter would conceivably filter out the expected high frequency measurement noise of an accelerometer, it would not provide regular position approximations to be used as a continuous reference. Thus, regardless of how slight the measurement error might be, due to the integration and the cumulative nature of obtaining subsequent positions, the error would grow over time without any mechanism in place to reduce it. The application of a Kalman Filter was therefore considered as it offers both the capability of reducing the error of actual measurements and the prediction of non-measurable system quantities in real time [31]. The motion of the point at which a sensor is mounted is subject to physical constraints formulated by the equations of motion. Based on such kinematic constraints and the known input, the Kalman filter makes a prediction as to the state of the structural element associated with the respective sensor as the next time step. The Kalman filter then evaluates this prediction by relating it to a measurement as it was obtained by the sensor itself. It then appropriates weights, representing the contribution of prediction and measurement to the final state estimate. The Kalman filter can be applied to any points of interest along the wing which is associated with one or multiple sensors. It can draw information from 
any number of contributing sensor sources to assure an optimal state estimate. For the case of estimating bending, the sensor cannot measure displacement explicitly. Therefore, in order for the Kalman filter to obtain the required displacement measurement, a structural model has to be developed. This model converts the sensor measurement into an implicit state measurement. The central emphasis of this dissertation is to further develop and inertial sensors system, investigating variations of methods for prediction and measurement and the inclusion of additional structural models which allow the sensing of more complex deformations. A detailed narrative of the process of using inertial sensors in combination with a Kalman filter is provided in the next chapter, which is followed by the illustration and discussion of preliminary and final results. 


\section{CHAPTER 3}

\section{KINEMATIC ANALYSIS METHOD}

Previously described methods provided an overview of different approaches to quantify wing deformation. The approach of utilizing MEMS sensor technology was found to be the most favorable method for further consideration and analysis. This section describes in detail the method of using micro-electronic accelerometers and gyroscopes in combination with a Kalman filter state estimator to estimate the deflection and torsion of an aircraft wing in real time. The project and associated development efforts were structured into stages with respective milestones which are discussed in this chapter.

\subsection{Kinematic Approach}

The kinematic approach formed the basis for a proof of concept and initial experimentation which were conducted with raw data obtained from an accelerometer and gyroscope. Because of some inherent limitations the scope of this approach was limited to obtaining static deflection of a structure. For the kinematic approach it was desired to show that data collected from microelectronic mechanical inertial sensors can be used to measure the static displacement of a point of interest from its original position. This concept can conceivably be expanded and applied to obtain static position of multiple points as they are displaced from their equilibrium position; such points then make up a three-dimensional object and the displacement of all combined points may represent the object's deformation. A variety of raw-data accelerometers and gyros were analyzed to obtain translational and rotational data. More accurate IMU motion sensors which incorporate a logic for sensor fusion of acceleration and gyration data were introduced later on to improve the performance with the enhanced finite element approach. In order to obtain real time acceleration and gyration, data acquisition methods and hardware were taken into consideration both of which are discussed in the subsequent section of this chapter. The aforementioned limitation of the kinematic approach can mostly be attributed to the static nature of the model which is to be used in order to assemble the state estimator. 
Nevertheless the dynamic nature of the system was captured by querying inertial sensors which provide motion rates and subsequently integrating the rates to obtain displacement. Starting with translational motion, acceleration can be integrated with respect to time once, to obtain the rate of displacement or velocity $v$. Integrating again yields the displacement $s$. Such quantities are respectively defined by the following system of equations:

$$
\begin{aligned}
\int a d t & =v_{0}+a t \\
\iint a d t d t & =s_{0}+v_{t}+\frac{1}{2} a_{t} \Delta t^{2}
\end{aligned}
$$

Similarly, equations can be constructed to describe rotational motion about a point $\mathrm{P}$. The absolute orientation angle $\phi$ is obtained by the sum of the previous orientation $\phi$ and the angular rate $\omega$ multiplied by the time step as shown in equation (3.3)

$$
\int \omega d t=\phi_{0}+\omega_{t} \Delta t
$$

Based on this relation, initial experiments were conducted to find displacement solely by integrating acceleration twice with respect to time. Similarly experiments were conducted to obtain torsion by integrating rotational data. The experimental setup and corresponding results are discussed in detail in later chapters. The use of pure integration did not yield the desired accuracy, showed to be very noisy and the displacement diverged quickly due to error accumulation during the double integration process and the lack of a reference position. This seems plausible because initial position and velocity are needed to compute subsequent values for position and velocity and over time the initial values become skewed. Integrating acceleration twice inevitably resulted in a large error build-up which was mentioned in previous sections and was also discussed in work done by Gilbert et al. [32]. Similarly, a gyroscope will experience drift over time causing it to become disoriented and hence requiring periodic calibration to 
maintain acceptable performance characteristics. In order to achieve a higher accuracy and avoid rapid error build up the use of a Kalman filter state estimator was considered.

\subsubsection{Kalman Filter and Sensor Fusion of kinematic and static sensor data}

Obtaining position solely from integrating motion rates proved to be an inadequate technique as sensor noise and uncertainty caused significant error build up over time. The Kalman filter has the advantage of filtering noisy sensor signals and furthermore combining data from various sources to obtain a more accurate estimate of the true system states [31]. In order to implement the Kalman filter state estimator two sources of information must be readily available, namely the prediction of the state at the next time step as well as the measurement of the state at that time. Based on the uncertainty of prediction and measurement, the most weight is given to the source with the least amount of uncertainty. The error covariance of process and measurement are essential to quantify and introduce uncertainty and error distribution. The prediction of the state is computed by referring to the kinematic equations of motion and applying the physical properties to the system states at the present time in order to predict the system states at the next time step.

\subsubsection{Simulation}

Prior to the development of the previously discussed sensor system, it was desired to simulate and test the Kalman filter and sensor fusion system design with parameters closely matching those of the actual system. A central motivation for running a simulation was to conceptualize and validate the system, create a path forward in the design and implementation of the Kalman filter and provide a platform to analyze the performance and characteristics of the actual sensor system in a later development phase. In order to validate the system concept of implementing a Kalman filter and fusing various sensor data for the proposed application, much consideration was given to the selection of the simulation environment. In particular, to assure the acquisition of realistic results, system and sensor parameters such as process and measurement noise characteristics, namely the associated standard deviation within the simulation must be equivalent to those expected from the actual system. The sensors were evaluated and performance 
parameters were used to assemble the covariance matrices for the simulation.

Initial simulations were conducted using only one accelerometer. More comprehensive simulations were expanded to include multiple sensors (accelerometers and gyroscopes). Analogous to the system properties discussed in the previous section the accelerometer serves as a dual-purpose data source. The raw acceleration provides the input to the physical system or plant. Its associated noise makes up the process noise and the respective standard deviation is used to assemble to covariance matrix of the process noise. Using the acceleration as an input to the system described by the kinematic EOM, a prediction of the new deflection can be made. In addition to the prediction the Kalman filter requires a measurement to compare its prediction against. The measurement is also obtained from the accelerometer and its ability to sense inherent exposure to gravity. Figure 3.2 shows that the tilt angle of the accelerometer can be obtained by decomposing the sensed gravity vector into its respective components of the sensor fixed local reference frame.

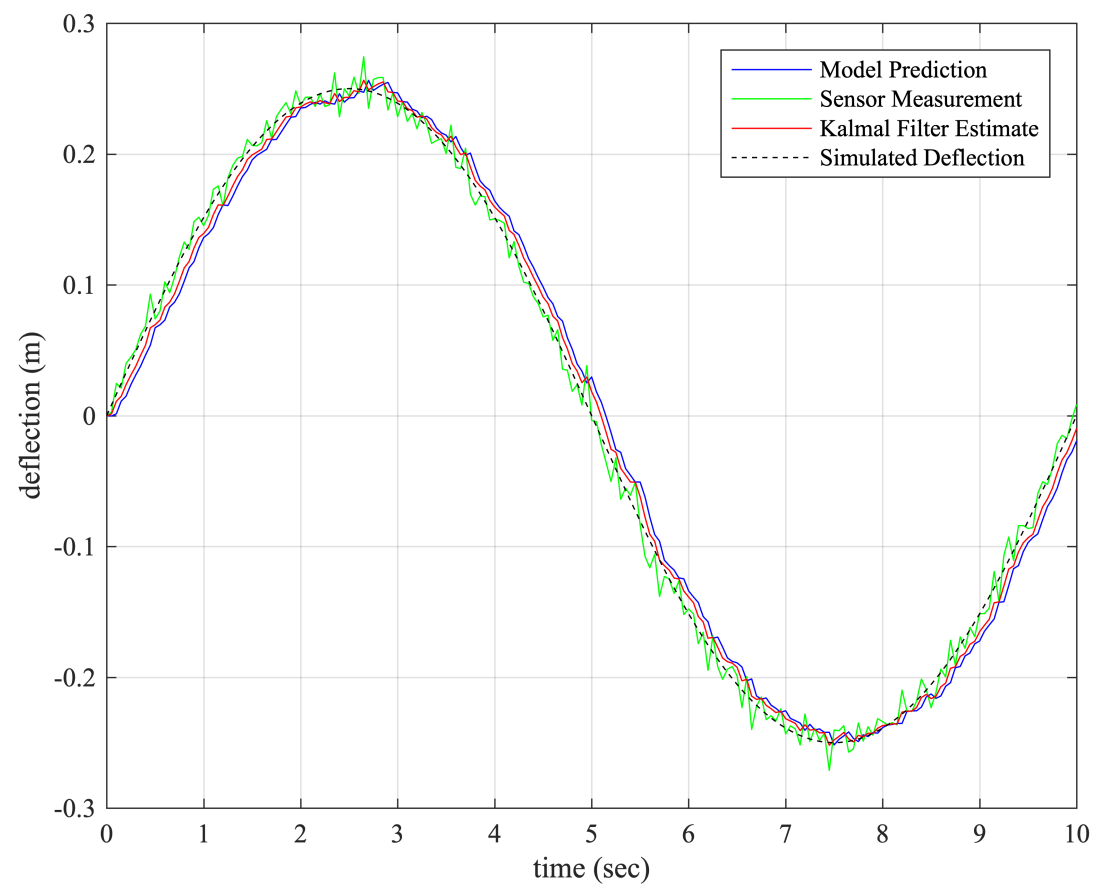

Figure 3.1: Simulation of Kalman Filter State Estimator 


\subsubsection{Bending}

To describe the translation of a point $\mathrm{P}$ at which an accelerometer is placed, equation (3.1) and equation (3.2) were discretized:

$$
\begin{aligned}
& s_{t+1}=s_{t}+\frac{v_{t+1}+v_{t}}{2} \Delta t \\
& v_{t+1}=v_{t}+a_{t} \Delta t \\
& s_{t+1}=s_{t}+v_{t} \Delta t+\frac{1}{2} a_{t} \Delta t^{2}
\end{aligned}
$$

A prediction of the new position will therefore be defined by the previous position, corrected for displacement contributions due to velocity and acceleration at the previous position and time. These equations represent the system or plant and can be re-arranged in order to obtain the state space representation of the sensor's motion equation (3.7), The two states are defined by the position $s$ and the velocity $v$. The system input is given by the acceleration $a$ as sensed by the accelerometer at point $\mathbf{P}$. Since the desired state for translational motion is position $s$, the output equation in state space representation is given by:

$$
x_{t+1}=\left[\begin{array}{c}
s_{t+1} \\
v_{t+1}
\end{array}\right]=\left[\begin{array}{cc}
1 & \Delta t \\
0 & 1
\end{array}\right]\left[\begin{array}{c}
s_{t} \\
v_{t}
\end{array}\right]+\left[\begin{array}{c}
\frac{1}{2} \Delta t^{2} \\
\Delta t
\end{array}\right] a_{t}
$$

Since the desired state for translational motion is position s, the output equation in state space representation is given by:

$$
z_{t}=\left[\begin{array}{ll}
1 & 0
\end{array}\right]\left[\begin{array}{l}
s_{t} \\
v_{t}
\end{array}\right]
$$

At each time step the Kalman filter uses the prediction and evaluates it with regards to the measurement. Sensors fusion occurs when the input data (acceleration) for prediction is driven by information obtained from one sensor whereas the measured data, in this case displacement, is 
collected from another sensor. When estimating bending however, it is challenging to obtain a measurement since the accelerometer is the only useful sensor capable of providing translational motion data. It delivers the acceleration input $u$ for the state prediction. Since the system input is comprised of the raw acceleration as sensed by the accelerometer it must be reduced by the gravitational component to derive only the dynamic portion or change in acceleration as seen in Figure 3.2 and equation (3.9).

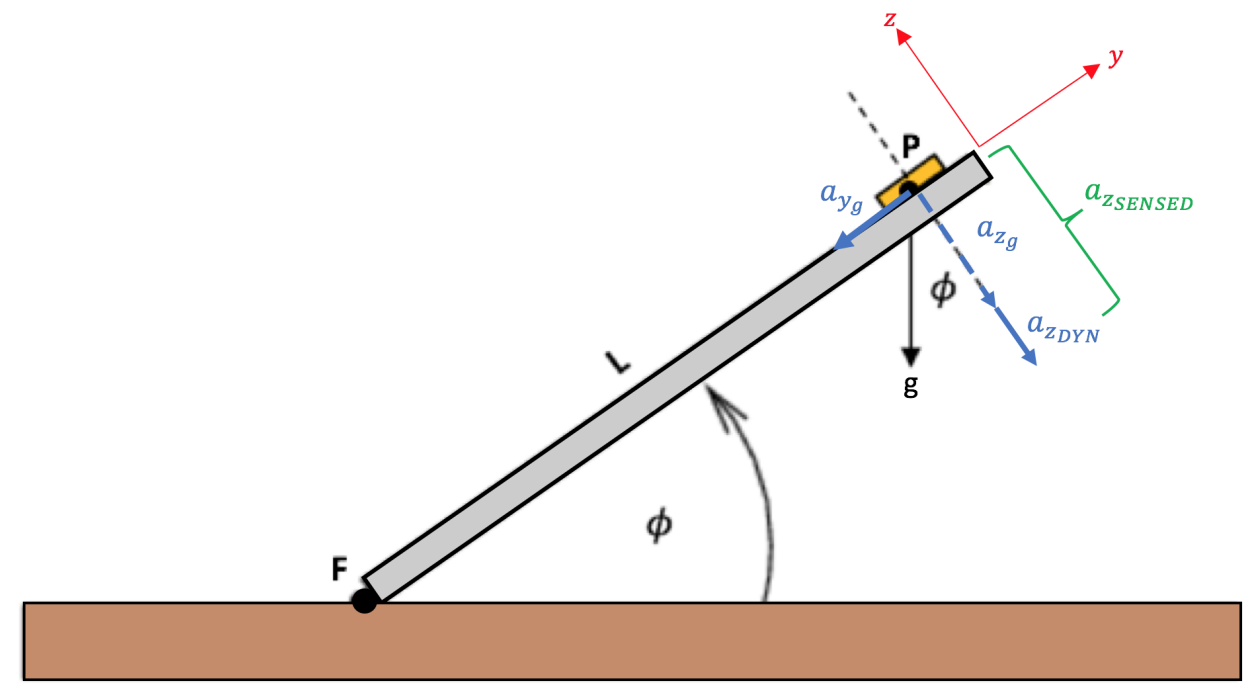

Figure 3.2: Schematic of pivoting beam

This dynamic portion $a_{Z}$ of the sensed acceleration is the input in the state space equation (3.7)

$$
a_{z_{D Y N}}=a_{z_{\text {sensed }}}-g \sin (\phi)
$$

To introduce a position measurement, the accelerometer can be used simultaneously to observe the gravity vector as a reference and relate the componential gravity to tilt angle information about all 3 axes. The measurement is therefore comprised of the displacement, measured implicitly by relating the sensed direction of the gravitational acceleration vector, expressed as the tilt angle $\phi$ to the actual displacement $\mathrm{h}$ of the accelerometer at point $\mathrm{P}$. This implicit measurement as shown in equation (3.10) is not a direct displacement measurement as it depends entirely on processing the 
structural model of the deformable component. The governing static structural model to obtain the measured displacement at any intermediate location $\mathrm{x}$ is defined by:

$$
h(x)=x \sin (\phi)
$$

A second proof of concept considers an experiment with a more complex structural model to incorporate static bending.

$$
h(x)=\frac{x(3 L-x) \phi}{(2 L-x)}
$$

The input to the dynamic system equation (3.7) does not change and continues to be the acceleration as sensed by the accelerometer. The system matrices are also not affected by the change in how the measurement is computed. Since the only modification to the system is the structural model that defines the measurement, all remaining system parameters except for the measurement noise covariance matrix remain unchanged. It should be noted that the measurement noise covariance is a sensor performance characteristic, in this case accelerometer specific and should conceivably remain constant. However, because of the implicit nature of relating acceleration to tilt angle and consequently deflection, depending on the structural model, the mathematical conversion can result in a shift or scaling of the error distribution and associated standard deviation. The standard deviation was obtained by collecting accelerometer data over time to determine the standard deviation. Figure 3.3 shows static bending under consideration of the structural model defined by equation (3.24). 


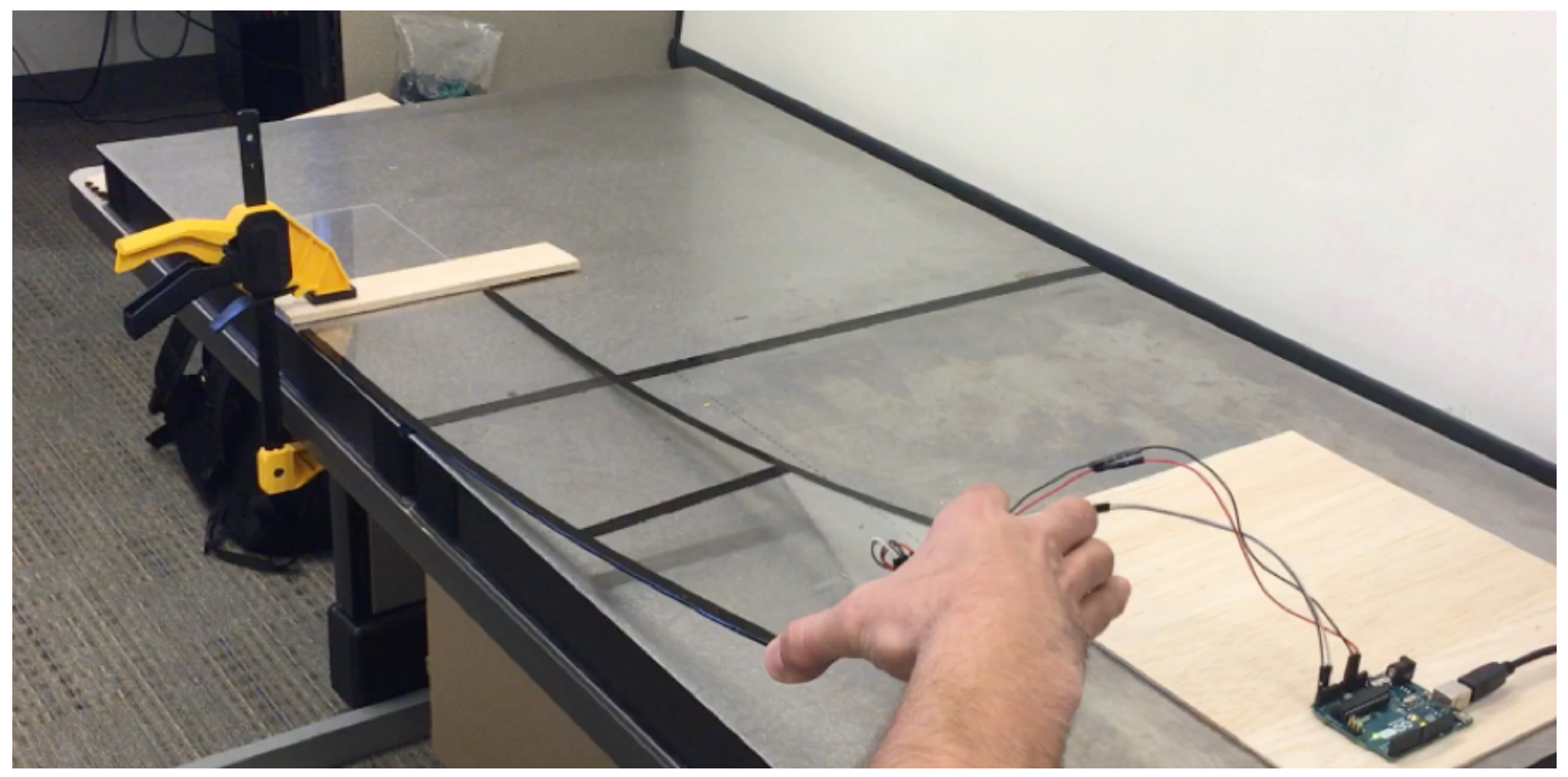

Figure 3.3: Wing deflection measurement using single accelerometer

\subsubsection{Torsion}

An extension of the above described methods to sense wing deflection is the inclusion of a gyroscope in order to gain knowledge about the torsion of the wing. The gyroscope senses angular rate of rotation which quantifies the rate of change in twist while the accelerometer measures the present twist angle by using the sensed gravity vector as a reference. To ensure reference data integrity in flight, this gravity vector would be obtained from reference sensors located on a rigid part of the aircraft like the fuselage. Similar to the accelerometer, the single equation of motion for the gyroscope is discretized equation (3.12) whereby the new orientation angle $\phi$ is the sum of the previous orientation and the turn rate $\omega$ multiplied by the lapsed time $\Delta t$.

$$
\theta_{t+1}=\theta_{t}+\omega \Delta t
$$

For the gyroscope an additional equation is added to incorporate the drift rate bias c, which is a sensor performance specific property and describes the gyroscopic precession over time; it is assumed to be constant. The system input is given by the angular rate $\omega$ as sensed by the gyroscope. 
The corresponding state space representation is therefor given by equation (3.13).

$$
x_{t+1}=\left[\begin{array}{c}
\theta_{t+1} \\
c_{t+1}
\end{array}\right]=\left[\begin{array}{cc}
1 & -\Delta t \\
0 & 1
\end{array}\right]\left[\begin{array}{c}
\theta_{t} \\
c_{t}
\end{array}\right]+\left[\begin{array}{c}
\Delta t \\
0
\end{array}\right] \omega
$$

The desired state for rotational motion state is the orientation angle $\theta$, the output equation in state space representation is therefore given by equation (3.14).

$$
z_{t}=\theta_{a}
$$

For the case of twist, chord wise buckling is assumed to be negligible and the direct measurement of twist precludes the need for a structural model. The Kalman filter design and incorporated sensor fusion to determine torsion by inferring rotational motion and orientation are more conventional than for the case of the pure bending. At each time step the states are predicted using the state space representation of the plant. The tilt rate $\omega$ as sensed by the gyroscope acts as the system input. The measurement comes from the accelerometer which measures orientation by decomposing the direction of gravity thereby compensating for the angular drift of the gyroscope. Figure 3.4 shows the profile view of a plate that is rotated.

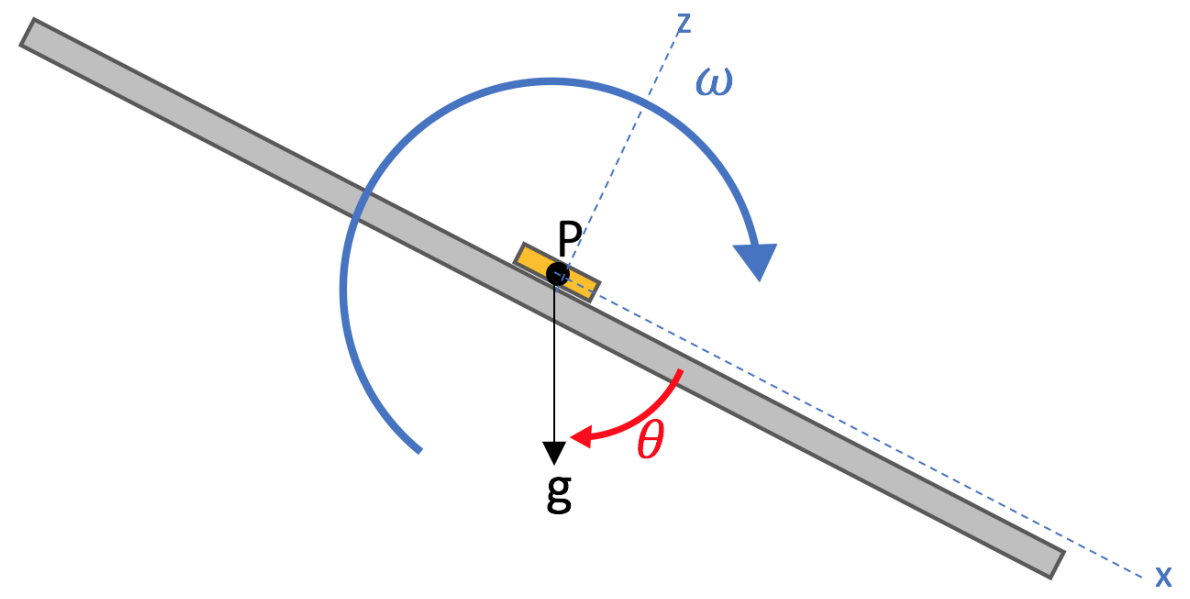

Figure 3.4: Torsion Measurement with Accelerometer and Gyro

A microchip containing two independent sensors, a gyroscope and an accelerometer is 
mounted at point $\mathrm{P}$. Both, angular and rate $\omega$ and gravity components as sensed by the accelerometer provide information on the orientation angle $\theta$. To complete the Kalman filter design, the remaining components needed are the covariance matrices $\mathrm{Q}$ and $\mathrm{R}$ for process and measurement noise respectively as well as the posteriori error covariance matrix P. Q and R are time invariant process and sensor specific matrices and correspond to the performance of the prediction and sensor measurement . Both matrices express the error distribution by referring to the standard deviation. $\mathrm{P}$, the posteriori error covariance matrix is time variant and converges over time to its steady state value. Equations (3.15) to (3.19) show the Kalman filter algorithm.

\section{Prediction}

$$
\begin{aligned}
& \hat{x}_{k+1}^{-}=A \hat{x}_{k}+B u_{k+1} \\
& P_{k+1}^{-}=A P_{k} A^{T}+Q
\end{aligned}
$$

\section{Measurement}

$$
\begin{aligned}
\mathcal{K}_{k+1} & =P_{k+1}^{-} H^{T}\left(H P_{k+1}^{-} H^{T}+R\right)^{-1} \\
\hat{x}_{k+1} & =\hat{x}_{k+1}^{-}+\mathcal{K}_{k+1}\left(z_{k+1}-H \hat{x}_{k+1}^{-}\right) \\
P_{k+1} & =\left(I-\mathcal{K}_{k+1} H\right) P_{k+1}^{-}
\end{aligned}
$$

Sensor specific performance characteristics were taken into consideration to obtain the appropriate covariance matrices based on the standard deviation for both bending and torsion. The sensor readings were collected at rest for the accelerometer and the gyroscope in order to obtain error distribution and the associated standard deviation. This quantity is sensor specific and varies among different sensor models. Based on the sensor's standard deviation the resulting covariance matrices 
for bending and torsion are shown in equations (3.20) to (3.23) respectively:

$$
\begin{aligned}
Q_{\text {Bending }} & =\left[\begin{array}{cc}
\sigma^{2} & 0 \\
0 & \sigma^{2}
\end{array}\right]=\left[\begin{array}{cc}
3.27 \cdot 10^{-4} & 0 \\
0 & 3.27 \cdot 10^{-4}
\end{array}\right] \mathrm{m}^{2} \\
R_{\text {Acc }} & =\left[\sigma^{2}\right]=4.49 \cdot 10^{-3} \frac{\mathrm{m}^{2}}{\mathrm{~s}^{4}} \\
Q_{\text {Torsion }} & =\left[\begin{array}{cc}
\sigma^{2} & 0 \\
0 & \sigma^{2}
\end{array}\right]=\left[\begin{array}{cc}
0.0106 & 0 \\
0 & 0.0106
\end{array}\right] \frac{\mathrm{rad}}{\mathrm{s}^{2}} \\
R_{\text {Gyro }} & =\left[\sigma^{2}\right]=4.9 \cdot 10^{-3} \frac{\mathrm{rad}^{2}}{\mathrm{~s}^{2}}
\end{aligned}
$$

The error covariance matrix $\mathrm{P}$ is expected to converge, however convergence time is not critical for the steady state performance, therefore the initial value for the error covariance, $P_{0}$ was set to be a 2x2 identity matrix equation (3.24) for both bending and torsion.

$$
P_{0}=\left[\begin{array}{cc}
\sigma^{2} & 0 \\
0 & \sigma^{2}
\end{array}\right]=\left[\begin{array}{ll}
1 & 0 \\
0 & 1
\end{array}\right]
$$

Substituting the corresponding matrices and values for the bending and torsion case into the general Kalman Filter routine equations (3.15) to (3.19) yield equations (3.25) to (3.29) and equations (3.30) to (3.34) respectively.

\section{Prediction (Bending)}

$$
\begin{aligned}
& {[\hat{s}]_{k+1}^{-}=\left[\begin{array}{cc}
1 & \Delta t \\
0 & 1
\end{array}\right]\left[\begin{array}{c}
s_{0} \\
v_{0}
\end{array}\right]+\left[\begin{array}{c}
\frac{1}{2} \Delta t^{2} \\
\Delta t
\end{array}\right] a_{k+1}} \\
& P_{k+1}=\left[\begin{array}{cc}
1 & \Delta t \\
0 & 1
\end{array}\right]\left[\begin{array}{cc}
1 & 0 \\
0 & 1
\end{array}\right]\left[\begin{array}{cc}
1 & \Delta t \\
0 & 1
\end{array}\right]^{T}+\left[\begin{array}{cc}
3.27 \cdot 10^{-4} & 0 \\
0 & 3.27 \cdot 10^{-4}
\end{array}\right]
\end{aligned}
$$




$$
\begin{aligned}
\mathcal{K}_{k+1} & =P_{k+1}^{-}\left[\begin{array}{ll}
1 & 0
\end{array}\right]^{T}\left([10] P_{k+1}^{-}\left[\begin{array}{ll}
1 & 0
\end{array}\right]^{T}+4.49 \cdot 10^{-3}\right)^{-1} \\
\hat{x}_{k+1} & =\hat{x}_{k+1}^{-}+\mathcal{K}_{k+1}\left(z_{k+1}-\left[\begin{array}{ll}
1 & 0
\end{array}\right] \hat{x}_{k+1}^{-}\right) \\
P_{k+1} & =\left(I-\mathcal{K}_{k+1}[10]\right) P_{k+1}^{-}
\end{aligned}
$$

\section{Prediction (Torsion)}

$$
\begin{aligned}
{\left[\begin{array}{l}
\hat{s} \\
\hat{v}
\end{array}\right]_{k+1}^{-} } & =\left[\begin{array}{ll}
1 & \Delta t \\
0 & 1
\end{array}\right]\left[\begin{array}{l}
s_{0} \\
v_{0}
\end{array}\right]+\left[\begin{array}{c}
\frac{1}{2} \Delta t^{2} \\
\Delta t
\end{array}\right] a_{k+1} \\
P_{k+1} & =\left[\begin{array}{ll}
1 & \Delta t \\
0 & 1
\end{array}\right]\left[\begin{array}{ll}
1 & 0 \\
0 & 1
\end{array}\right]\left[\begin{array}{cc}
1 & \Delta t \\
0 & 1
\end{array}\right]^{T}+\left[\begin{array}{cc}
0.0106 & 0 \\
0 & 0.0106
\end{array}\right] \\
\mathcal{K}_{k+1} & =P_{k+1}^{-}\left[\begin{array}{ll}
1 & 0
\end{array}\right]^{T}\left([10] P_{k+1}^{-}\left[\begin{array}{ll}
1 & 0
\end{array}\right]^{T}+4.9 \cdot 10^{-3}\right)^{-1} \\
\hat{x}_{k+1} & =\hat{x}_{k+1}^{-}+\mathcal{K}_{k+1}\left(z_{k+1}-\left[\begin{array}{ll}
1 & 0
\end{array}\right] \hat{x}_{k+1}^{-}\right) \\
P_{k+1} & =\left(I-\mathcal{K}_{k+1}[10]\right) P_{k+1}^{-}
\end{aligned}
$$

where the state estimate $\hat{x}$ is comprised of the orientation $\theta$ and the angular drift bias $\mathrm{c}$.

After successful implementation of both bending and torsion, both motions were combined in separate Kalman routines. A custom Matlab ${ }^{\circledR}$ visualization script displays the behavior of the flexible plate upon bending and twist. This script breaks the plate structure up into panels. Each plate has four points with $\mathrm{x}, \mathrm{y}$ and $\mathrm{z}$ coordinates and a virtual centerline position which is equidistant to leading and trailing edge. The structural model and displacement estimates are used 
to determine the vertical displacement of the centerline. Using the torque estimate the panels are subsequently rotated to allow the real time display of bending and torsion. Figure 3.7 in the next chapter on experimental testing illustrates the setup and digital visualization of the deformation.

\subsection{Hardware and Software Implementation}

For the development of a system as previously described multiple sensor types and models were considered. In order to assure flexibility and simple interfacing between the computer and the sensor, it was desired to find sensor hardware which would be compatible with a versatile microcontroller such as the Arduino Uno Figure 3.5a The advantage of the Arduino Uno is the well documented, open source software and hardware environment. The heart of the controller is an 8-bit, $16 \mathrm{MHz}$ ATmega328 processor. An I2C serial bus in conjunction with a multiplexer Figure 3.5 b allows for up to 8 sensors to be connected per multiplexer breakout board. Up to 8 multiplexer boards may be connected per Arduino Uno resulting in a theoretical operation of up to 64 sensors.

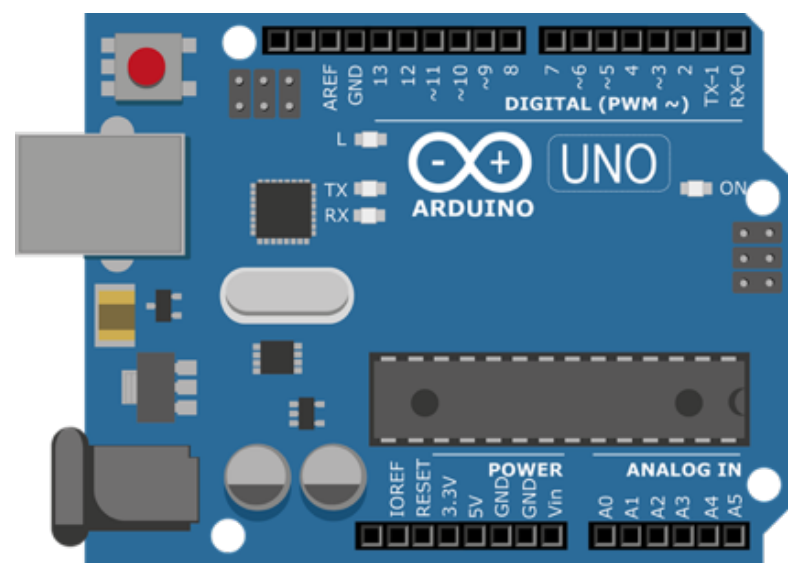

(a) Arduino Uno Microcontroller

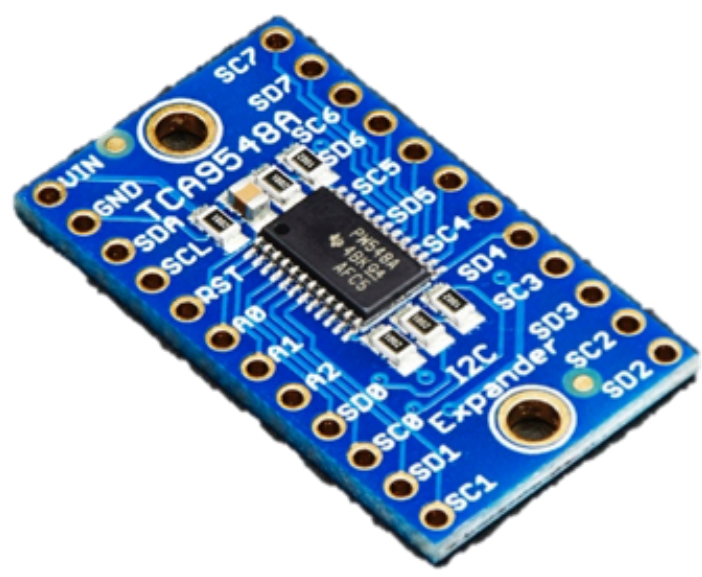

(b) TCA9548A Multiplexer

Figure 3.5: Hardware components

Advancements in microchip technology allow for mechanical sensors to be scaled down to a few millimeters, while maintaining reasonable accuracy, full functionality, and reliable sensor outputs. These components are also referred to as microelectromechanical system (MEMS) sensors. The relevant MEMS sensors include an accelerometer and a gyroscope, which provide 
raw data of translational acceleration and angular velocity, respectively. Inertial sensors present the key ingredient for the herein described concept of a quantifying aircraft wing deformation. Initial experiments and proof of concept were conducted using an inexpensive three axis accelerometer and magnetometer LSM303 module and later switched to a higher accuracy 9-DOF dual independent accelerometer, gyroscope breakout board (FXOS8700/FXAS21002). The sensors used in the structural analysis Chapter 5 are BNO055 9-DOF IMU motion processors which combine accelerometer, gyro and magnetometer data to compute Euler angles. Figure 3.6b shows the a close up of the MEMS accelerometer akin to the one used for this project. Unlike conventional spring-loaded accelerometers, MEMS sensors relates acceleration to the displacement of a proof mass. When the wing is statically or dynamically deflected, displacement and gravity results in a deflection of capacitive half bridges and hence a change in capacitance. This capacitive differential is detected, amplified, and converted to a digital output signal, which can then be utilized as input to the Kalman filter. Figure 3.6a show the combined accelerometer and gyroscope breakout board that was used for most experiments.

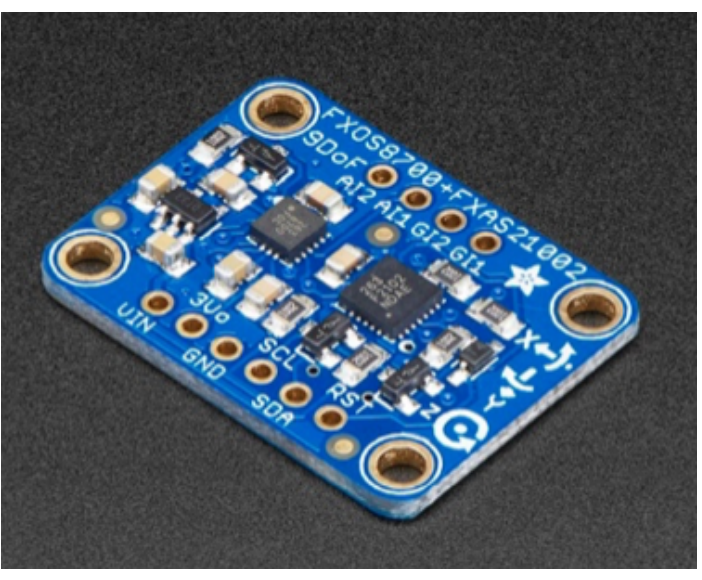

(a) gyro/accelrometer breakout

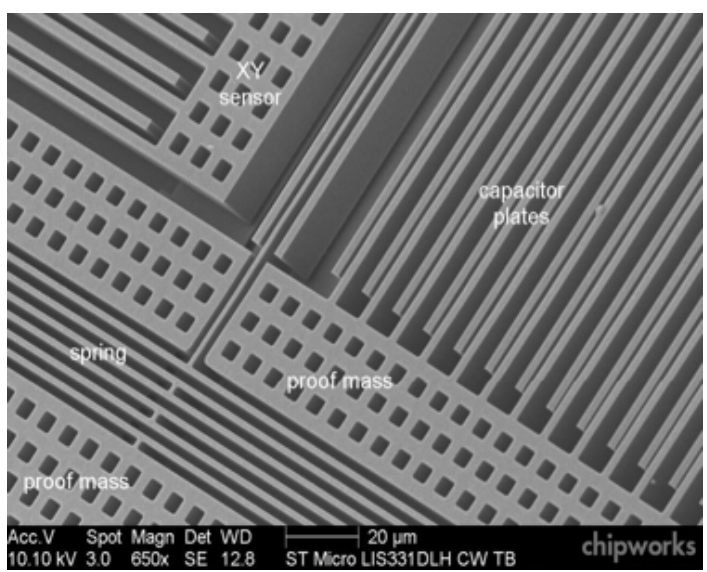

(b) LIS331DLH teardown

Figure 3.6: Sensor close-up and tear down (courtesy of Adafruit Inc. and Chipworks Inc.

Unlike the accelerometer or magnetometers, gyroscope does not measure external inputs such as gravity or magnetic fields. Gyroscopes detect angular velocity based on the device rotation. It should be noted that MEMS gyroscopes such as the ones used for this project do not exhibit an actual rotation. Instead they contain rapidly oscillating MEMS structures. When the gyroscope is 
subjected to a rotation, the oscillating structures are stimulated orthogonally by the Coriolis force. Capacitive electrodes can sense the resulting vibration and relate it to angular rates.

A data collection and processing routine in $\mathrm{C}++$ was written for the Arduino microcontroller to read the data from the various inertial sensors. Initially the Kalman filtering was also performed on the microcontroller level by a separate modular routine but was moved to Matlab for simplicity and overall flexibility as it allowed a process to more effectively analyze the information. The Arduino Uno monitors all connected sensors. Whenever Matlab performs an iteration it queries the Uno and collects and transmits a current sweep of all sensors. Only then will a data array consisting of raw gyro and accelerometer data be sent to Matlab via a serial connection for further processing. This handshake helps to avoid caching old data and assures proper synchronization. These new values for the system input and measurement are supplied to the state estimation routine. Each set of data is then sent to Matlab via a serial connection where the discussed Kalman filter routines are applied. A detailed result analysis of experimental testing to this point will be discussed in the next chapter.

\subsection{Preliminary Testing}

Preliminary testing of the kinematic approach was conducted. This included several simple experiments of single accelerometers to detect displacement. This chapter will discuss the preliminary testing and discussion of the corresponding results. Figure 3.7 shows a calibrated deflection of a beam about a fulcrum $F$, This experiment was conducted with a 3-axis accelerometer, connected to an Arduino Uno micro-controller. This experiment served as a proof of concept as it captures the motion of a pivoting beam. The rigid beam of length L pivots uniformly about $F$. The accelerometer serves two purposes: it captures the gravity vector which can be related to the tilt angle $\phi$ as described in Section 3.1.3, equation (3.10), It also measures the acceleration to provide as input for the kinematic equations. The beam was subjected to a continuous oscillatory deflection, constrained to calibrated peak deflections. The results of this rudimentary experiment will be discussed on Chapter 4. 


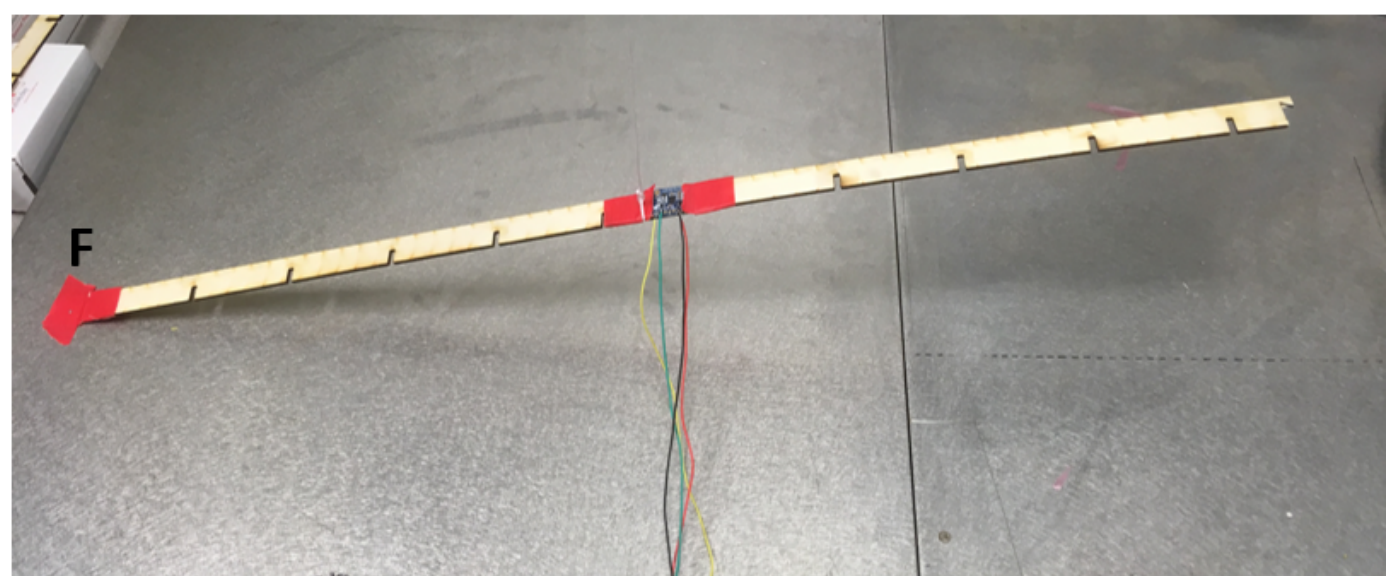

Figure 3.7: Pivoting beam with single 3-axis accelerometer

An extension of this experiment was conducted with the use of an accelerometer in conjunction with a gyroscope to obtain information on structural torsion. The limitations of the current setup were discussed previously. Figure 3.8 shows the combined use of accelerometer and gyroscope data to quantify wing deformation after the Kalman filter was applied. A real-time visualization script uses the static bending model equation (3.11) to convert the structural deformation data from the Kalman state estimate into deflection data across the structure. The inset of Figure 3.8 illustrates this deflection in a 3-D real time animation.

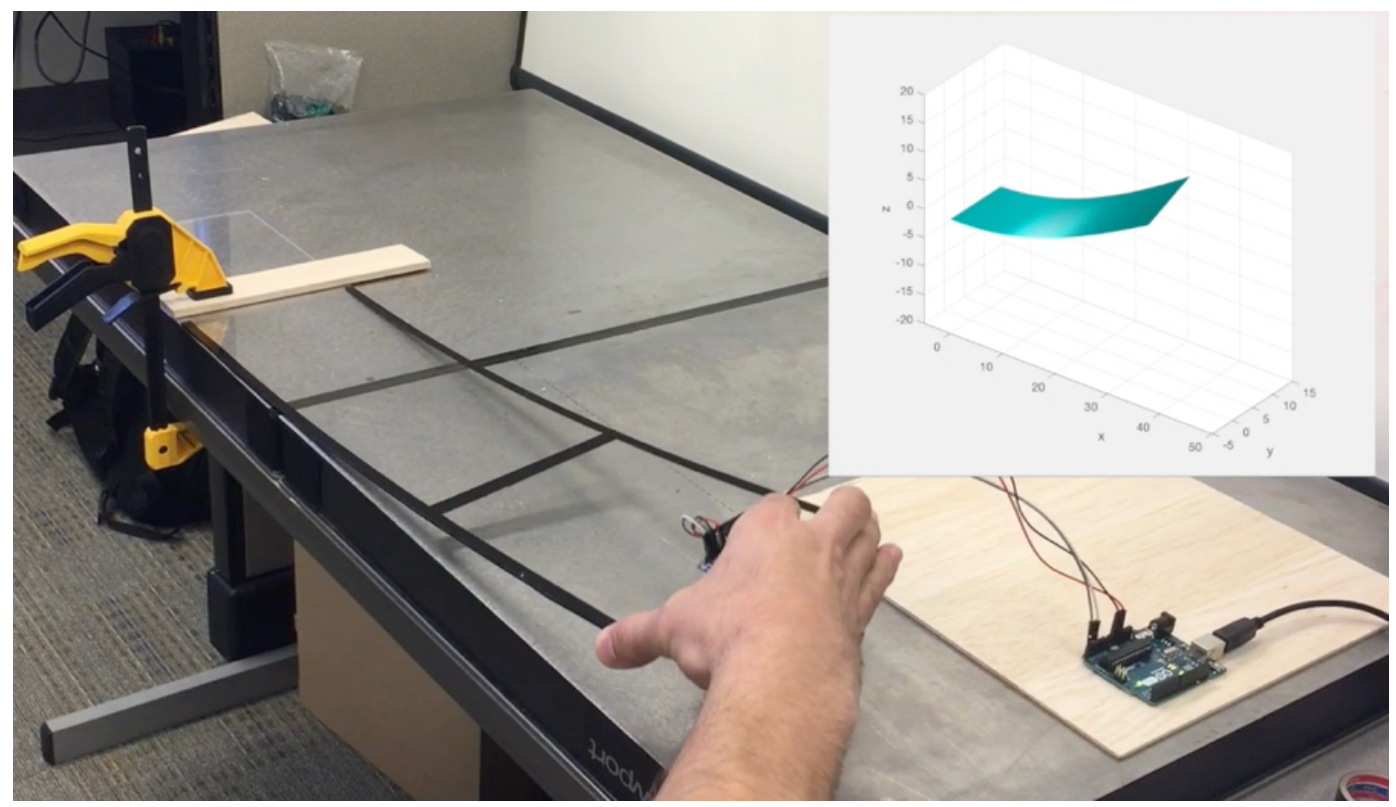

Figure 3.8: Torsion Measurement with Accelerometer and Gyro 
The testing method described above provide a conclusive overview of the MEMS sensors capabilities and limitations. Keeping in mind that the preliminary testing serves primarily as a proof of concept, it is a valid assertion that such methods were lacking some ability to adequately validate results from the state estimator and properly identify the limitations of the kinematic approach.The next chapter describes a new approach of utilizing MEMS sensors in conjunction with a finite element model to address the aforementioned limitations and achieve higher accuracy in the results. 


\section{CHAPTER 4}

\section{RESULTS AND DISCUSSION - PART 1: KINEMATIC APPROACH}

Preliminary experiments were conducted with the experimental setup and methodology described in the previous chapter. The corresponding results of the initial kinematic approach are discussed in this chapter. The method of using inertial MEMS sensors required a detailed sensor analysis to explore and identify the performance characteristics and limitations. Various sensor models for accelerometer and gyroscope were considered for use and tested individually. The preliminary results presented and discussed in this section are confined to the Adafruit FXOS8700 accelerometer and FXAS21002 gyroscope, the sensors which yielded the most favorable performance and applicability and which were used for kinematic approach.

\subsection{Position tracking using only accelerometer data}

One of the first experiments conducted was based on the notion of computing the displacement and absolute position of the accelerometer by using pure integration of acceleration and velocity from the previous times step over the lapsed time. We recall that the simple discrete system 4.1 describes the absolute position of the accelerometer starting from rest based on cumulative change in position

$$
s_{t+1}=s_{t}+v_{t} \Delta t+\frac{1}{2} a_{t} \Delta t^{2}
$$

To examine the error build-up as a result of continuously integrating acceleration the concept was applied to an experiment of a pivoting beam Figure 3.2. The displacement of the beam was determined solely by referencing velocity and position information of the previous time step and integration of the latter and raw acceleration (Eq. 4.1).

The following graph (Fig. 4.1) shows this concept applied to the pivoting beam to obtain the position purely by integrating the sensed acceleration $a_{s}$ twice and adding it to the previous position to obtain displacement $s_{a}$ (blue curve). The displacement $s_{m}$ (amber curve) is obtained by 
relating the tilt angle derived from sensor measurement to the deflection using the gravity vector as a reference. The sensor was displaced from its' neutral position upward by $0.25 \mathrm{~m}$ with every

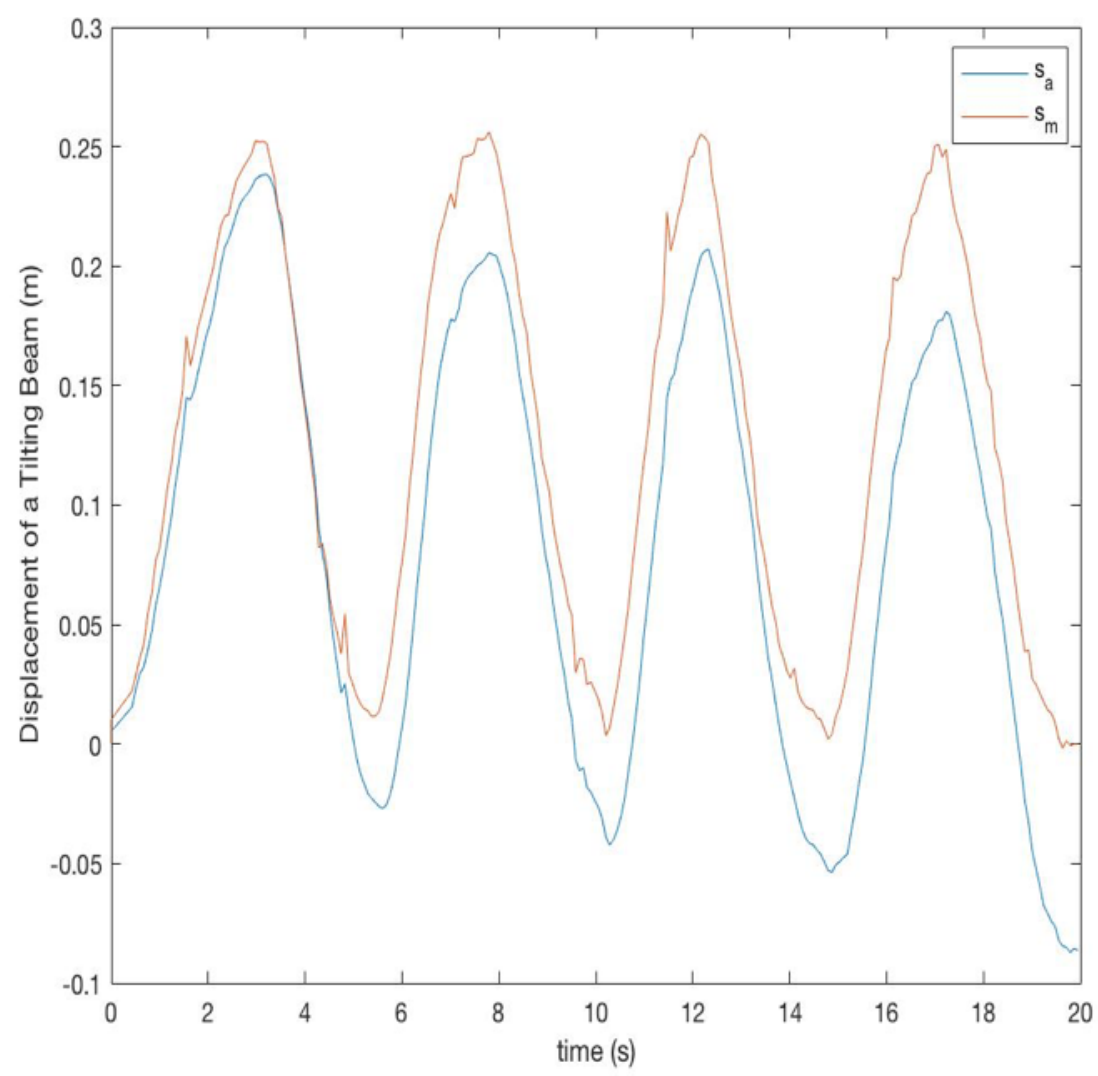

Figure 4.1: Displacement based on integrating raw acceleration

tilt cycle 3.7. The data shows that the integrated raw acceleration progressively undershoots the actual deflection. The cumulative integration error buildup is very noticeable in this experiment as time passes. The measurement $s_{m}$ shows more reliable but noisy values for the deflection $\mathrm{s}$.

\subsection{Gyroscope Performance Data}

Analogous to the accelerometer, preliminary testing of the gyroscope provided performance characteristics of the sensor and in order to to quantify the required error distribution for the state estimator. Similarly, we can recall that the angular displacement can be obtained 
using a simple kinematic relationship (Eq. 4.2).

$$
\phi_{t+1}=\omega_{t}+\phi_{t} \Delta t
$$

Fig. 30 shows the angular rates integrated over time and added to the angle at the previous time step (Eq. 4.2 for a gyroscope at rest. The figure illustrates the change in orientation and the gyroscopic precession over time when at rest without any external disturbances. It is apparent that

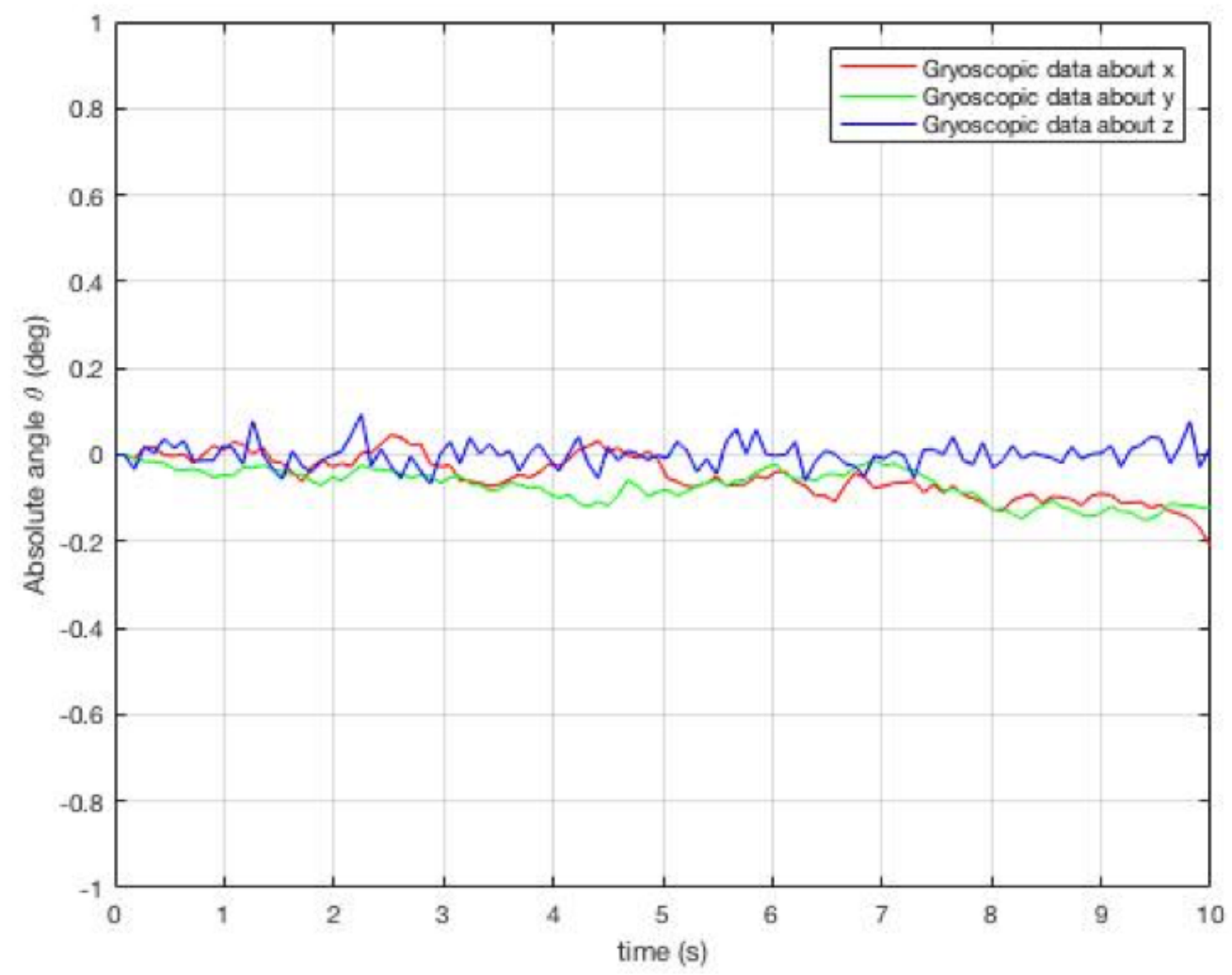

Figure 4.2: Gyroscopic Precession

the gyroscope exhibits some precession and integration induced drift even when held at rest of approximately $0.02 \mathrm{deg} / \mathrm{sec}$. The angular drift grew significantly when the gyroscope was moved and then returned to its original position. 


\subsection{Kinematic Approach with Kalman Filter Implementation}

Because of the aforementioned inaccuracies of both accelerometer and gyroscope a Kalman filter state estimator was first introduced with appropriate sensor specific parameters to estimate the bending and torsion of a test structure. Measurements were derived by relating sensed gravity to the required tilt angle. To measure bending the tilt angle was converted using the appropriate structural model while for torsion, the tilt angle itself is the measurement. A flexible flat plate was equipped with an accelerometer and a gyroscope. Calibrated bending and twist motions were performed and recorded with and without the use of the Kalman filter. The implementation of the Kalman filter state estimator improved the accuracy and noise of the displacement output significantly. Figure 4.3 compares the Kalman state estimate of the displacement (blue line) to the measurement (green line) and the prediction (red line) for a flexible flat plate structure which was deflected in 3 cyclic bending motions constrained to approximately 0.2 meters which is shown by the red dashed line. The estimate shown in blue shows an aggregate of the measurement and the prediction. As time goes by and the first cycle is completed, the displacement measurement in the second and third peak shows a decreasing trend while the prediction remains close to the dashed deflection limits. The lower values for sensor measurements however affects the state estimate. Some of the measurement undershoot can be attributed to improper or inaccurate deflection rather than as well as error build up as part of the integration.

Similar results were observed for the case of the twist/torsion as seen in Figure 4.4 Kalman Filter for Torsion. The Kalman filter was capable of estimating the torsion angle $\phi$ and omitting noise from measurement and prediction. The angular estimate was accurate to $4^{\circ}$ during a cyclic torsion of $30^{\circ}$ to each side.

\subsection{Discussion}

Both gyroscope and accelerometer show a high level of accuracy when it comes to tracking raw acceleration and angular rates of rotation. The absolute position of the sensor at rest after double integrating the raw acceleration shows some anticipated error accumulation. This in turn would negatively affect the prediction accuracy; when tracking absolute position while the 


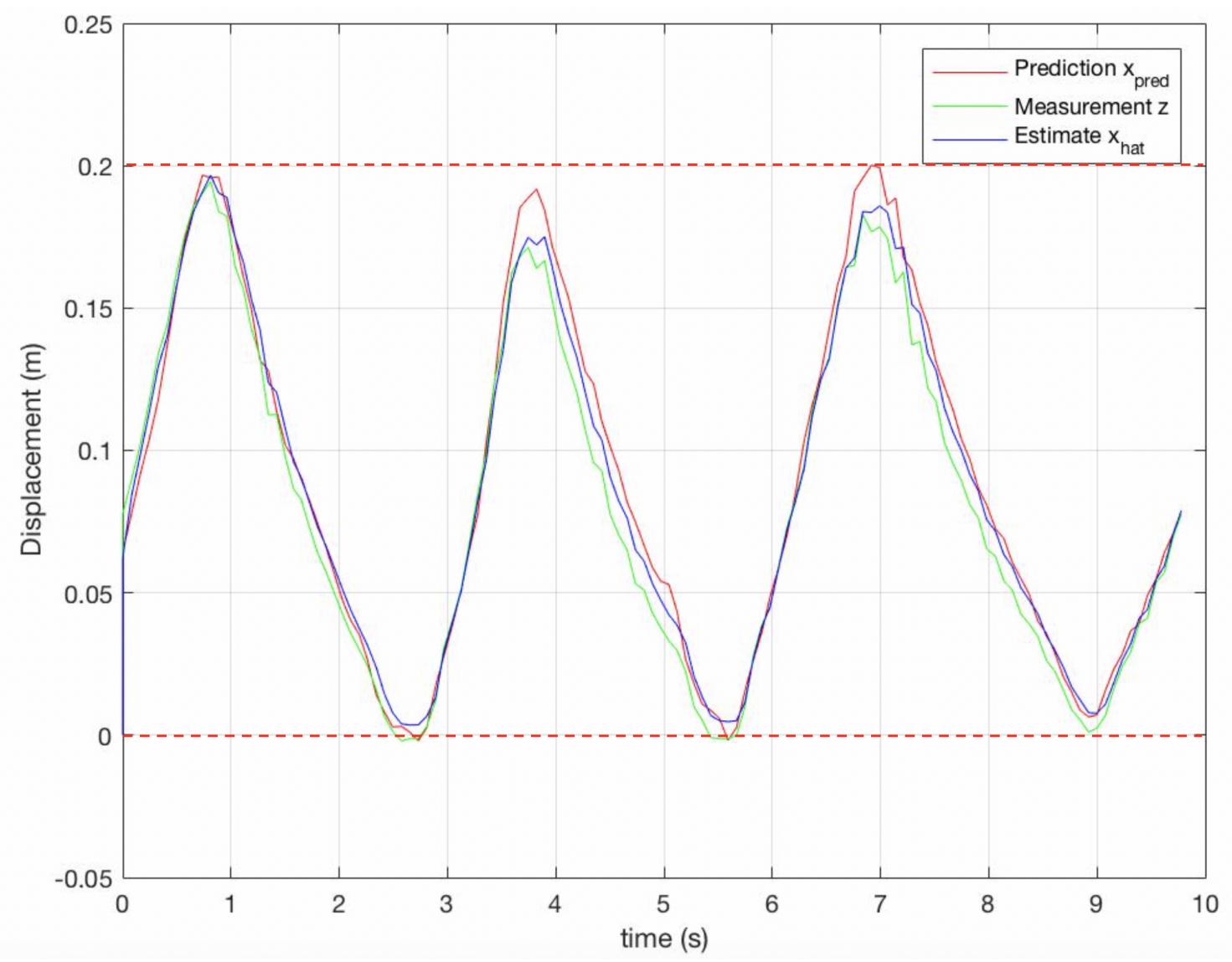

Figure 4.3: Kalman filter for bending

sensor is in motion, the accelerometer tends to exhibit more of the expected cumulative error and consequentially undergoes hysteresis because the neutral starting position prior to the initial displacement is never re-captured. The results drawn from the preliminary testing of the Kalman Filter system design and associated experimental setup show the state estimator to behave desirably, supporting the proof of concept and further development of the system . The algorithm determines the most probable state of the system based on measurement and prediction. Compared to the simulation however, the measurement and the prediction show a greater discrepancy than expected. A plausible cause for this could be a residual gravitational component affecting the dynamic portion of the acceleration, hence skewing the prediction noticeably.

\subsubsection{Limitations of the Kinematic Approach}

Preliminary testing revealed that application of the Kalman filter delivers desirable results, however, when applied to the kinematic approach is limited to pseudo dynamic, slow motion 


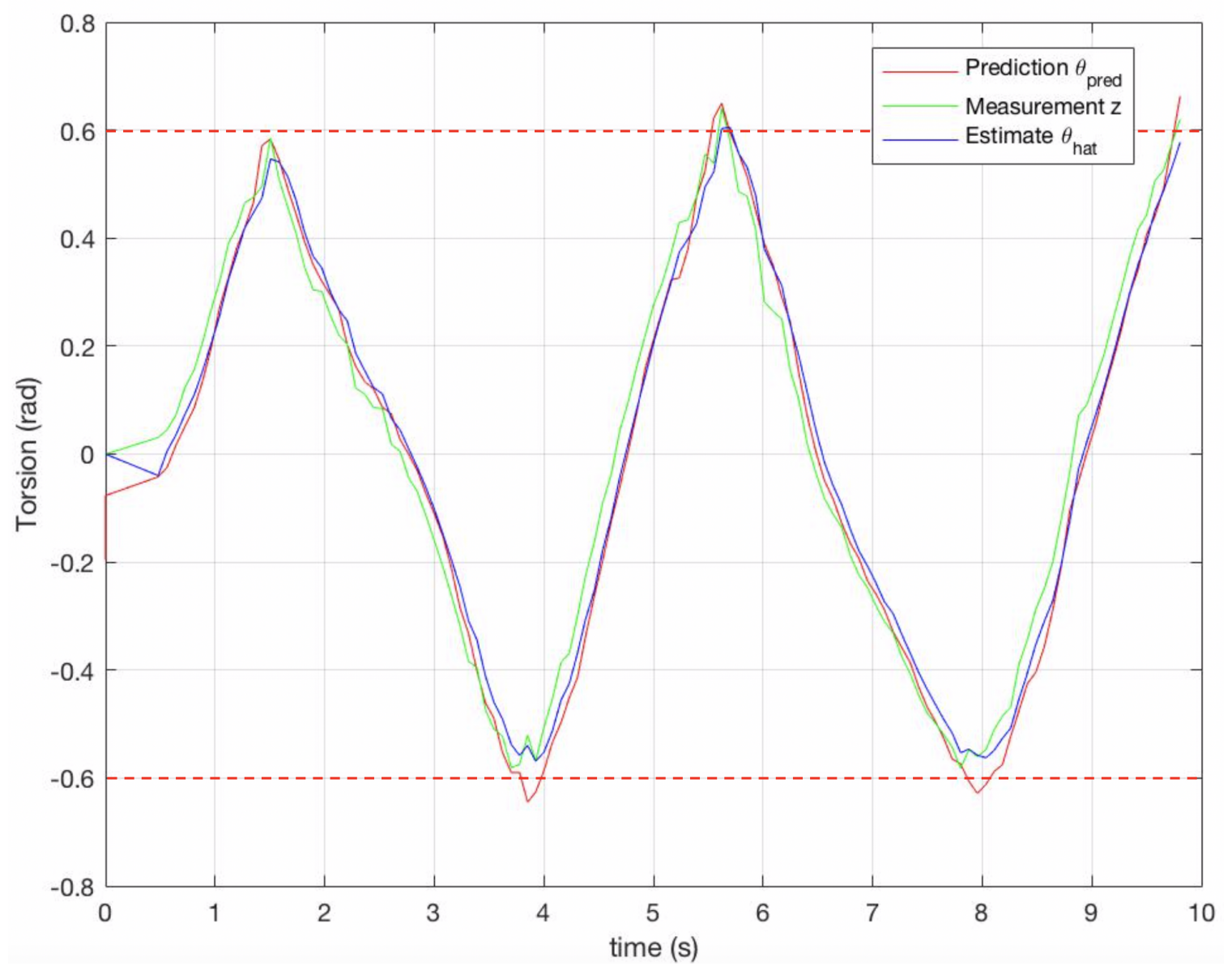

Figure 4.4: Kalman Filter for Torsion

deflection. Even though testing was conducted using dynamic bending and torsion with satisfactory results, we recall that the structural model used to convert the measurements currently does not support qualitative statement of dynamic deflection. Higher accuracy can be achieved when including a dynamical model for a structure to estimate various higher order bending modes. This approach along with additional proposals for improvement will be discussed in the next chapter. A method description of the advanced structural analysis and associated experimental tests will be provided, followed by detailed results and validation for the finite element approach and the respective experimental testing procedures. 


\section{CHAPTER 5}

\section{STRUCTURAL DYNAMICS ANALYSIS METHOD}

To incorporate the dynamic behavior of the flat plate two methods were examined but first it is important to recapitulate the significance of measurement and prediction for the Kalman filter state estimator. We recall that for the previously discussed approach the equations of motion of a particle were considered to obtain a prediction for the position at the next time step. One can recall that the state of interest namely the vertical displacement depends on the position and velocity of the sensor at the previous time step. Acceleration obtained from the accelerometer then serves as system input. The measurement also depends on acceleration, obtained from the same accelerometer source whereby the acceleration is used to compute the tilt angle based on gravity. When substituted into the structural model, vertical displacement can subsequently be solved for. As discussed previously, this technique consequently relies on one sensor for both prediction as well as measurement and poses the concern of data dependence between the prediction and the measurement. More importantly the structural model equation (3.11) from chapter 3 used to describe the deformation is a static beam equation limited to first order bending under static or quasi static loading. In order to capture the motion of the flat plate structure a method must be developed to correctly describe the dynamic nature of the structure. For this we begin by looking at a simple case of a bending beam and recall that the bending of such a beam is described by the following differential equation equation (5.1) also known as the Euler Bernoulli beam equation:

$$
\frac{\partial^{2}}{\partial x^{2}}\left(E I \frac{\partial^{2} w}{\partial x^{2}}\right)=-\mu \frac{\partial^{2} w}{\partial t^{2}}+Q
$$

Analogously the dynamics of a flat plate can be described in a similar fashion by an extension of the Euler Bernoulli equation valid for a thin flat plate structure equation (5.2),

$$
\rho h \ddot{w}+\mathcal{D} \nabla^{4} w=Q
$$


where $\mathrm{Q}$ is the externally applied loads and $\mathcal{D}$ is the flexural rigidity:

$$
\mathcal{D}=\frac{E_{A} h^{3}}{12\left(1-v^{2}\right)}
$$

\subsection{Finite Difference method}

Starting with the partial differential equation describing the flat plate equation (5.2), it is the objective to use real time sensor data and obtain an expression of the vertical displacement $w$. Just by inspection one can easily identify the 2 nd order time derivative $\frac{\partial^{2} w}{\partial t^{2}}=a_{z}$ as the vertical acceleration which we can conveniently obtain from accelerometer data. The spatial derivatives however cannot so easily be replaced with sensor data but must rather be expressed as a function of $w$, which in turn it is desired to solve for. This can be accomplished using a finite difference scheme, which allows the decomposition of higher order derivatives into a lower order difference equations based on a Taylor series expansion. For simplicity it was first assumed that deformation

occurs only along the span of the wing. Thus $\frac{\partial^{4} w}{\partial x^{4}}=0$ and $\frac{\partial^{4} w}{\partial x^{2} \partial y^{2}}=0$. Then equation (5.2) reduces to:

$$
\rho h \frac{\partial^{2} w}{\partial t^{2}}+\mathcal{D} \frac{\partial^{4} w}{\partial y^{4}}=0
$$

Note that the first order derivative of $w$ is the bending angle $\phi$ :

$$
\frac{\partial w}{\partial y}=\phi
$$

Thus we can reduce the order of the derivative:

$$
\frac{\partial^{4} w}{\partial y^{4}}=\frac{\partial^{3} \phi}{\partial y^{3}}
$$


and

$$
\rho h \frac{\partial^{2} w}{\partial t^{2}}+\mathcal{D} \frac{\partial^{3} \phi}{\partial y^{3}}=Q
$$

We can now express equation equation (5.5) by a combination of finite difference equations, more specifically backward, central and forward difference equations (5.7) to (5.9),

$$
\begin{aligned}
f^{\prime \prime \prime}\left(x_{0}\right) & =\frac{1 \phi x_{0}-3 \phi x_{-1}+3 \phi x_{-2}-1 \phi_{-3}}{\Delta x^{3}} \\
f^{\prime \prime \prime}\left(x_{0}\right) & =\frac{-\frac{1}{2} \phi x_{-2}+\phi x_{-1}+3 \phi x_{+1}+\frac{1}{2} \phi_{+2}}{\Delta x^{3}} \\
f^{\prime \prime \prime}\left(x_{0}\right) & =\frac{-1 \phi x_{0}+3 \phi x_{+1}-3 \phi x_{+2}+1 \phi_{+3}}{\Delta x^{3}}
\end{aligned}
$$

Figure 5.1 illustrates how the finite difference equations can be used to form a system of equations to describe the vertical displacement at each sensor location. The number of sensors required depends on the order of desired accuracy and the order of the derivative at $x_{0}$. In turn the choice of finite difference formulation depends on the number of available neighboring sensors. Thus, for the $4^{\text {th }}$ order spatial derivative at least 6 sensors are required to adequately approximate the solution.

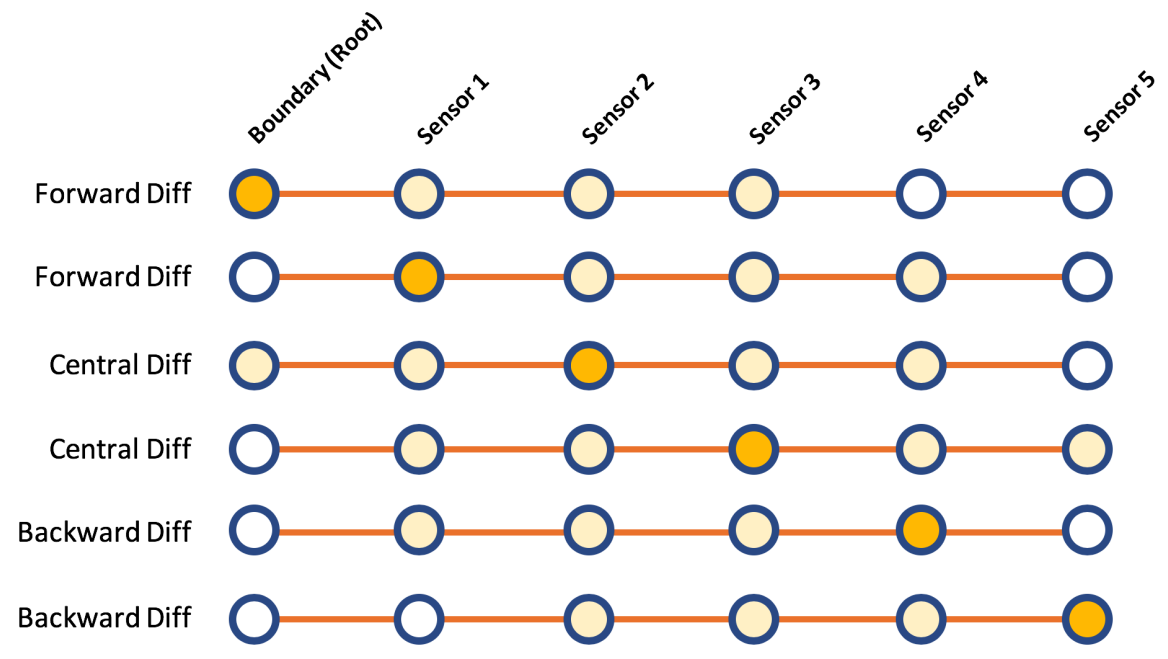

Figure 5.1: Finite Difference Scheme 
equation (5.6) can now be expressed by the coefficient matrix multiplied by the sensor position vector and the sensed acceleration $a_{z}$.

$$
\rho h a_{z}+\frac{D}{\Delta x^{3}}\left[\begin{array}{cccccc}
-1 & 3 & -3 & 1 & 0 & 0 \\
0 & -1 & 3 & -3 & 1 & 0 \\
-\frac{1}{2} & 1 & 0 & -1 & \frac{1}{2} & 0 \\
0 & -\frac{1}{2} & 1 & 0 & -1 & \frac{1}{2} \\
0 & 1 & -3 & 3 & -1 & 0 \\
0 & 0 & 1 & -3 & 3 & -1
\end{array}\right]\left[\begin{array}{l}
\phi_{1} \\
\phi_{2} \\
\phi_{3} \\
\phi_{4} \\
\phi_{5} \\
\phi_{6}
\end{array}\right]=Q
$$

Subsequently solving for the deflection angle vector $\phi$ and subsequently approximating the deflection $w$ piecewise would now appear a mere trivial task, this however is not the case. Inspection of the coefficient matrix shows that given the combination of finite difference equations results in a singular and hence non-invertible matrix. Numerical experimentation and manipulation with the various combinations of finite difference equations and the inclusion of additional sensors yielded no satisfactory outcome in that the coefficient matrix remained of singular nature. Further investigation showed a linear dependence between pairs of expressions for the respective sensors. Consequently, an inversion of the coefficient matrix is not easily possible. Furthermore, the finite difference approach would not solve the concern of data dependence between the measurement and the prediction as both would still rely on the same sensor source. It was therefore decided to investigate alternate means to obtain a prediction for the flat plate deformation.

\subsection{Finite Element Approach}

Since the finite difference approximations was neither easily implementable nor did it yield the desired compatibility with the given problem, a different approach was considered that could adequately capture the dynamic behavior of the flat plate. The finite element method (FEM) was examined as an approximate analytical solution to the partial differential equation equation (5.1), FEM is an effective, sensor independent numerical modeling method to describe 
the physical properties and dynamic behavior of a structure analytically, which can oftentimes be difficult or cumbersome to determine with exact analytical solution techniques. The premise of the finite element method is based on the idea of analyzing a structure piece-wise rather than as a whole, while assuring that the governing equations of motion within and across neighboring elements and the applicable boundary conditions of the structure are satisfied. This section will outline the process of applying the finite element method to the given dynamic system of a flat plate wing structure. For a more generalized explanation of converting a differential equation into a finite element model and the associated derivations the reader should refer to the steps outlined in [33]. A similar case of developing the equations of motion for a flat plate with a trailing edge flap was investigated in previous work by Menon et. al. [6].

\subsubsection{Prediction}

Prior to discussing the steps involved in obtaining a finite element model we shall reiterate the goal of this process, namely the development of a dynamic model of the wing structure, which will serve as a numerical prediction for the deformation of the wing in order to assemble the Kalman state estimator. Derivations leading to the partial differential equation expressing the equations of motion of the flat plate can be found in previous work by [34]. For our case the wing structure is comprised of a uniform flat plate with known material properties which is clamped on one side as shown in Figure 5.2

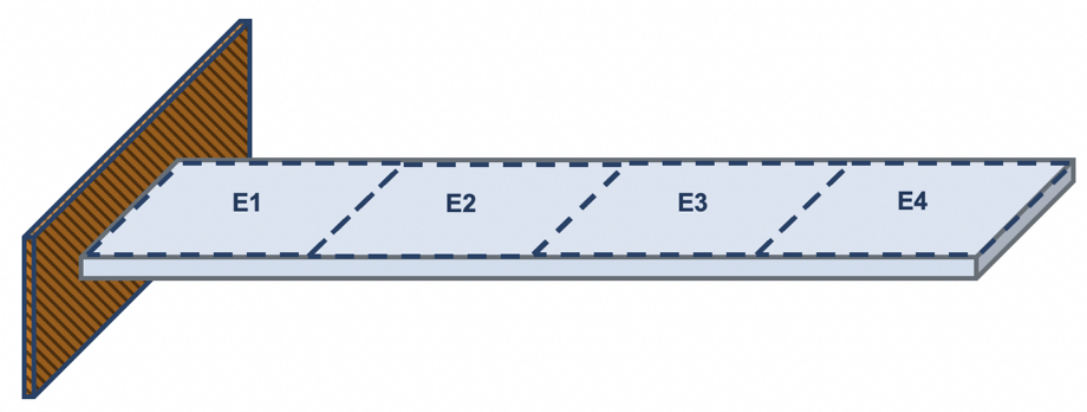

Figure 5.2: FEM grid geometry

Each element is described by a simple geometry; for this case the wing structure was divided into 4 square elements. We consider the partial differential equation (5.2) and apply 
Galerkin's method. This method is based on the variational form and principle of weighted residuals whereby the error residual $R$ of an approximate solution to the differential equation is forced to vanish. Equation (5.2) becomes the residual R:

$$
R=\rho h \ddot{w}+\mathcal{D} \nabla^{4} w+L
$$

Doing so permits the strong form of the partial differential equation equation (5.2) to be converted into the weak form and an ordinary differential equation. This is accomplished by distributing the differential and shifting part of the fourth order partial derivative to from $w$ to the weight function $\psi$, thereby widening the range of admissible approximation shape functions for the approximation based on differentiability and continuity requirements. First, we multiply the error residual $\mathrm{R}$ with a set of weight functions $\psi$ equation (5.11) and integrating the residual across the entire surface.

$$
\int_{0}^{c} \int_{0}^{l} \psi\left(\rho h \ddot{w}+\mathcal{D} \nabla^{4} w+L\right) d x d y=0
$$

Derived from the product rule of integration we begin by examining the following single integral relationship describing the integration by parts:

$$
\int_{a}^{b} \psi \frac{w}{d x}=[\psi w]_{a}^{b}-\int_{0}^{l} \frac{d \psi}{d x} w d x
$$

for a $2^{\text {nd }}$ order differential equation we consider this expression:

$$
\begin{array}{rlrl}
\int_{a}^{b} \psi \frac{d^{2} w}{d x^{2}} d x & =\int_{0}^{l} \psi \frac{d}{d x}\left(\frac{d w}{d x}\right) d x & \\
& =\int_{0}^{l} \psi \frac{d v}{d x} d x, \quad \text { where } \quad v=\frac{d w}{d x}
\end{array}
$$


referring to equation (5.12) we obtain:

$$
\begin{aligned}
\int_{a}^{b} \psi \frac{d^{2} w}{d x^{2}} d x & =-\int_{a}^{b} v \frac{d \psi}{d x} d x+[\psi v]_{b}-[\psi v]_{a} \\
& =-\int_{a}^{b} \frac{d w}{d x} \frac{d \psi}{d x} d x+\left[\psi \frac{d w}{d x}\right]_{b}-\left[\psi \frac{d w}{d x}\right]_{a}
\end{aligned}
$$

which can be rewritten as

$$
-\int_{a}^{b} \frac{d w}{d x} \frac{d \psi}{d x} d x=\int_{a}^{b} \psi \frac{d^{2} w}{d x^{2}} d x+\left[\psi \frac{d w}{d x}\right]_{b}-\left[\psi \frac{d w}{d x}\right]_{a}
$$

Similarly we can apply this to the $4^{\text {th }}$ order spatial derivative inside of the residual $R$ from equation (5.11) after rearranging the terms:

$$
\begin{array}{r}
\int_{0}^{l} \int_{0}^{c} \psi_{i}\left(\rho h \ddot{w}+\mathcal{D} \nabla^{4} w+L\right) d x d y=0 \\
\int_{0}^{l} \int_{0}^{c} \psi_{i}(\rho h \ddot{w}) d x d y+\int_{0}^{l} \int_{0}^{c} \psi_{i} L d x d y+\mathcal{D} \int_{0}^{l} \int_{0}^{c} \psi_{i}\left(\nabla^{4} w\right) d x d y=0
\end{array}
$$

The Laplace operator if the $4^{\text {th }}$ order spatial derivative con now be expanded:

$$
\begin{aligned}
\mathcal{D} \int_{0}^{l} \int_{0}^{c} \psi_{i}\left(\nabla^{4} w\right) d x d y=\mathcal{D} & \int_{0}^{l} \int_{0}^{c} \psi_{i}\left(\frac{d^{4} w}{d x^{4}}\right) d x d y+2 \mathcal{D} \int_{0}^{l} \int_{0}^{c} \psi_{i}\left(\frac{d^{4} w}{d x^{2} d y^{2}}\right) d x d y \\
& +\mathcal{D} \int_{0}^{l} \int_{0}^{c} \psi_{i}\left(\frac{d^{4} w}{d y^{4}}\right) d x d y
\end{aligned}
$$

The process of obtaining the weak form is demonstrated on the inner integral and is also discussed in work by [33]

$$
\begin{aligned}
\mathcal{D} \int_{0}^{c} \psi_{i} \frac{d^{4} w}{d x^{4}} d x & =\mathcal{D} \int_{0}^{c} \psi_{i} \frac{d^{2}}{d x^{2}}\left(\frac{d^{2} w}{d x^{2}}\right) d x \\
& =\mathcal{D} \int_{0}^{c} \psi_{i} \frac{d^{2} u}{d x^{2}} d x, \quad \text { where } \quad u=\left(\frac{d^{2} w}{d x^{2}}\right)
\end{aligned}
$$


analogously to equation (5.15), equation (5.19) can be expressed as:

$$
\mathcal{D} \int_{0}^{c} \psi_{i} \frac{d^{4} w}{d x^{4}} d x=-\mathcal{D} \int_{a}^{b} \frac{d u}{d x} \frac{d \psi}{d x} d x+\mathcal{D}\left[\psi \frac{d u}{d x}\right]_{b}-\mathcal{D}\left[\psi \frac{d u}{d x}\right]_{a}
$$

based on the previously derived expression for the second order differential equation equation (5.16) we can rewrite equation (5.20).

$$
\begin{aligned}
& \mathcal{D} \int_{0}^{c} \psi_{i} \frac{d^{4} w}{d x^{4}} d x=\mathcal{D} \int_{a}^{b} u \frac{d^{2} \psi}{d x^{2}} d x+\mathcal{D}\left[u \frac{d \psi}{d x}\right]_{c}-\mathcal{D}\left[u \frac{d \psi}{d x}\right]_{0} \\
&+D\left[\psi_{i}\left(\frac{d u}{d x}\right)\right]_{c}-\mathcal{D}\left[\psi_{i}\left(\frac{d u}{d x}\right)\right]_{0}
\end{aligned}
$$

substituting $u=\left(\frac{d^{2} w}{d x^{2}}\right)$ and re-introducing the double integral yields:

$$
\mathcal{D} \int_{0}^{l} \int_{0}^{c} \psi_{i} \frac{d^{4} w}{d x^{4}} d x=\mathcal{D} \int_{0}^{l} \int_{0}^{c} \frac{d^{2} w}{d x^{2}} \frac{d^{2} \phi}{d x^{2}} d x d y+\mathcal{D} \int_{0}^{l}\left[\frac{d^{2} w}{d x^{2}} \frac{d \psi}{d x}\right]_{0}^{c} d y+\mathcal{D} \int_{0}^{l}\left[\psi_{i}\left(\frac{d^{3} w}{d x^{3}}\right)\right]_{0}^{c} d y
$$

Similarly the integration by parts can be performed on the $4^{\text {th }}$ order derivative in $y$-direction

$$
\mathcal{D} \int_{0}^{l} \int_{0}^{c} \psi_{i}\left(\frac{d^{4} w}{d x^{4}}\right) d x d y
$$

as well as the coupled derivative

$$
2 \mathcal{D} \int_{0}^{l} \int_{0}^{c} \psi_{i}\left(\frac{d^{4} w}{d x^{2} d y^{2}}\right) d x d y
$$

from equation (5.18) to yield:

$$
\begin{aligned}
\mathcal{D} \int_{0}^{l} \int_{0}^{c} \psi_{i} \frac{d^{4} w}{d y^{4}} d x d y=\mathcal{D} & \int_{0}^{l} \int_{c}^{0} \frac{d^{2} w}{d y^{2}} \frac{d^{2} \psi}{d y^{2}} d x d y \\
& +\mathcal{D} \int_{0}^{c}\left[\frac{d^{2} w}{d y^{2}} \frac{d \psi}{d y}\right]_{0}^{l} d x+\mathcal{D} \int_{0}^{c}\left[\psi_{i}\left(\frac{d^{3} w}{d y^{3}}\right)\right]_{0}^{l} d x
\end{aligned}
$$


and the sum of:

$$
\begin{aligned}
\mathcal{D} \int_{0}^{l} \int_{0}^{c} \psi_{i} \frac{d^{4} w}{d x^{2} d y^{2}} d x d y=\mathcal{D} & \int_{0}^{l} \int_{0}^{c} \frac{d^{2} w}{d y^{2}} \frac{d^{2} \psi}{d x^{2}} d x d y+\mathcal{D} \int_{0}^{l}\left[\frac{d^{2} w}{d y^{2}} \frac{d \psi}{d x}\right]_{0}^{c} d y \\
& +\mathcal{D} \int_{0}^{l}\left[\psi_{i}\left(\frac{d^{3} w}{d x d y^{2}}\right)\right]_{0}^{c} d y
\end{aligned}
$$

and

$$
\begin{aligned}
\mathcal{D} \int_{0}^{l} \int_{0}^{c} \psi_{i} \frac{d^{4} w}{d x^{2} d y^{2}} d x d y=\mathcal{D} & \int_{0}^{l} \int_{0}^{c} \frac{d^{2} w}{d x^{2}} \frac{d^{2} \psi}{d y^{2}} d x d y+\mathcal{D} \int_{0}^{c}\left[\frac{d^{2} w}{d x^{2}} \frac{d \psi}{d y}\right]_{0}^{l} d x \\
& +\mathcal{D} \int_{0}^{c}\left[\psi_{i}\left(\frac{d^{3} w}{d x^{2} d y}\right)\right]_{0}^{l} d y
\end{aligned}
$$

We now add equations (5.22) and (5.25) to (5.27).

$$
\begin{aligned}
\mathcal{D} \int_{0}^{l} \int_{0}^{c} \psi_{i}\left(\nabla^{4} w\right) d x d y=\mathcal{D} & \int_{0}^{l} \int_{0}^{c} \frac{d^{2} w}{d x^{2}} \frac{d^{2} \psi}{d x^{2}}+\frac{d^{2} w}{d y^{2}} \frac{d^{2} \psi}{d x^{2}}+\frac{d^{2} w}{d y^{2}} \frac{d^{2} \psi}{d y^{2}}+\frac{d^{2} w}{d x^{2}} \frac{d^{2} \psi}{d y^{2}} d x d y \\
& +\mathcal{D} \int_{0}^{l}\left[\frac{d^{2} w}{d x^{2}} \frac{d \psi}{d x}+\psi_{i}\left(\frac{d^{3} w}{d x^{3}}\right)+\frac{d^{2} w}{d y^{2}} \frac{d \psi}{d x}+\psi_{i}\left(\frac{d^{3} w}{d x d y^{2}}\right)\right]_{0}^{c} d y \\
& +\mathcal{D} \int_{0}^{c}\left[\frac{d^{2} w}{d y^{2}} \frac{d \psi}{d y}+\psi_{i}\left(\frac{d^{3} w}{d y^{3}}\right)+\frac{d^{2} w}{d x^{2}} \frac{d \psi}{d y}+\psi_{i}\left(\frac{d^{3} w}{d x^{2} d y}\right)\right]_{0}^{l} d x
\end{aligned}
$$

Adding and subtracting $2 \nu$ multiplied by equations (5.26) and (5.27) results in:

$$
\begin{aligned}
\mathcal{D} \int_{0}^{l} \int_{0}^{c} \psi_{i}\left(\nabla^{4} w\right) d x d y=- & \mathcal{D} \int_{0}^{l} \int_{0}^{c}\left(\frac{d^{2} w}{d x^{2}}+\nu \frac{d^{2} w}{d y^{2}}\right) \frac{d^{2} \psi}{d x^{2}}+\left(\frac{d^{2} w}{d y^{2}}+\nu \frac{d^{2} w}{d x^{2}}\right) \frac{d^{2} \psi}{d y^{2}} d x d y \\
& -\mathcal{D} \int_{0}^{l} \int_{0}^{c} 2(1-\nu) \frac{d^{2} w}{d x d y} \frac{d^{2} \psi}{d x d y} d x d y \\
& -\mathcal{D} \int_{0}^{l}\left[\frac{d^{3} w}{d x^{3}}+(2-\nu) \frac{d^{3} w}{d x d y^{2}}\right]_{0}^{c} \psi-\left[\left(\frac{d^{2} w}{d x^{2}}+\nu \frac{d^{2} w}{d y^{2}}\right) \frac{d \psi}{d x}\right]_{0}^{c} d y \\
& -\mathcal{D} \int_{0}^{c}\left[\frac{d^{3} w}{d y^{3}}+(2-\nu) \frac{d^{3} w}{d x^{2} d y}\right]_{c}^{l} \psi-\left[\left(\frac{d^{2} w}{d y^{2}}+\nu \frac{d^{2} w}{d x^{2}}\right) \frac{d \psi}{d y}\right]_{0}^{l} d x
\end{aligned}
$$


The relationships between moment, shear force and displacement was discussed by [34] and is given by.

$$
\begin{array}{ll}
M_{x}=-\mathcal{D}\left(\frac{d^{2} w}{d x^{2}}+\nu \frac{d^{2} w}{d y^{2}}\right) & V_{x}=-\mathcal{D}\left[\frac{d^{3} w}{d x^{3}}+(2-\nu) \frac{d^{3} w}{d x d y^{2}}\right] \\
M_{y}=-\mathcal{D}\left(\frac{d^{2} w}{d y^{2}}+\nu \frac{d^{2} w}{d x^{2}}\right) & V_{y}=-\mathcal{D}\left[\frac{d^{3} w}{d y^{3}}+(2-\nu) \frac{d^{3} w}{d x^{2} d y}\right] \\
M_{x y}=-\mathcal{D}(1-\nu) \frac{d^{2} w}{d x d y} &
\end{array}
$$

thus resulting in

$$
\begin{aligned}
\mathcal{D} \int_{0}^{l} \int_{0}^{c} \psi_{i}\left(\nabla^{4} w\right) d x d y= & -\mathcal{D} \int_{0}^{l} \int_{0}^{c} M_{x} \frac{d^{2} \psi}{d x^{2}}+M_{y} \frac{d^{2} \psi}{d y^{2}} d x d y+2 M_{x y} \frac{d^{2} \psi}{d x d y} d x d y \\
& -\mathcal{D} \int_{0}^{l}\left[V_{x} \psi-M_{x} \frac{d \psi}{d x}\right]_{0}^{c} d y-\mathcal{D} \int_{0}^{c}\left[V_{y} \psi-M_{y} \frac{d \psi}{d y}\right]_{0}^{l} d x
\end{aligned}
$$

Based on the physical properties of of the clamped flat plate the boundary conditions are defined as follows:

$$
\begin{array}{cc}
w_{y=0}=0 & \left(w_{y}\right)_{y=0}=0 \\
{\left[V_{x}\right]_{x=0}=0} & {\left[M_{x}\right]_{x=0}=0} \\
{\left[V_{x}\right]_{x=c}=0} & {\left[M_{x}\right]_{x=c}=0} \\
{\left[V_{y}\right]_{y=l_{1}}=0} & {\left[M_{y}\right]_{y=l_{1}}=0}
\end{array}
$$

We can substitute expressions for the moments and shear forces allowing us to reduce equation (5.30),

$$
\int_{0}^{b} \int_{0}^{c} M_{x} \psi_{x x}+M_{y} \psi_{y y}+2 M_{x y} \psi_{x y} d x d y=0
$$

When substituting equation (5.31) into equation (5.11) we obtain:

$$
\int_{0}^{b} \int_{0}^{c}\left[(\rho h \ddot{w}) \psi-\left(M_{x} \psi_{x x}+M_{y} \psi_{y y}+2 M_{x y} \psi_{x y}\right)+L \psi\right] d x d y=0
$$

Let us assume that $W$ is the exact solution to the plate equation equation (5.2) and introduce an 
approximation, referred to as trial or test function $w(x, y)$ :

$$
W \approx w(x, y)=\sum_{i=1}^{N} c_{i}(t) \phi_{i}(x, y)
$$

which can be substituted into equation (5.32).

$$
\int_{0}^{b} \int_{0}^{c}\left[\left(\rho h \ddot{c}_{i} \phi_{i}\right) \psi_{j}-\left(M_{x_{i}} \psi_{x x_{j}}+M_{y_{i}} \psi_{y y_{j}}+2 M_{x y_{i}} \psi_{x y_{j}}\right)+L \psi_{j}\right] d x d y=0
$$

Note that we can group the integral based on three physical qualities of the system and the associated coefficients, namely the stiffness, the damping and the mass of each element.

$$
\begin{aligned}
M_{i j} & =\int_{0}^{b} \int_{0}^{c} \rho h \psi_{i} \psi_{j} d x d y \\
D_{i j} & =L \int_{0}^{b} \int_{0}^{c} \psi_{i} \psi_{j} d x d y \\
K_{i j} & =-\int_{0}^{l_{1}} \int_{0}^{c}\left(M_{x} \psi_{x x_{j}}+M_{y} \psi_{y y_{j}}+2 M_{x} y \psi_{x y_{j}}\right) d x d y
\end{aligned}
$$

equation (5.34) can now be expressed in terms of the mass, damping and stiffness matrices as $2^{\text {nd }}$ order ordinary differential equation:

$$
M \ddot{c}+D \dot{c}+K c=0
$$

The paper and literature previously referenced limits the expression of the lift force L to selfinduced lift based on the vertical flapping motion shown in equation 18.

$$
L=C_{L_{\alpha}} \frac{\dot{w}}{U_{1}} \bar{q} S
$$

Analogously $\dot{w}$ is replaced by the approximation $\dot{c}_{i} \psi_{i}$. Externally induced lift based on non-zero free stream such as observed in flight will be introduced later in this chapter.

The physical behavior of each element can be approximately described by a set known 
shape or interpolation functions $\phi_{i}$. For Galerkin's method the set of weight functions consists of the same set as the shape functions used to approximate the physical behavior of the system such that

$$
\psi_{i}=\phi_{i}
$$

For the given wing structure the Hermite cubic conforming element [33] and its' associated equations equation (5.41a) - equation (5.41c) are assumed to be an adequate selection of shape functions.

$$
\begin{aligned}
\phi_{i} & =\frac{1}{8}\left(\xi_{0}+1\right)\left(\nu_{0}+1\right)\left(2+\xi_{0}+\nu_{0}-\xi^{2}-\nu^{2}\right) \\
\phi_{i+1} & =\frac{1}{8} \xi_{i}\left(\xi_{0}+1\right)^{2}\left(\xi_{0}-1\right)\left(\nu_{0}+1\right) \\
\phi_{i+2} & =\frac{1}{8} \nu_{i}\left(\xi_{0}+1\right)\left(\nu_{0}+1\right)^{2}\left(\xi_{0}-1\right)
\end{aligned}
$$

The respective mass, stiffness and damping matrices (M, K and D) for the master element were assembled such that the matrices at nodes which are shared between elements assure that the continuous and 'smooth' physical behavior of both neighboring elements is satisfied. The state transition and input matrices equation (5.42), equation (5.43), obtained fromequation (5.38), make up the state space representation and describe the dynamic behavior of the flat plate structure:

$$
\begin{gathered}
A=\left[\begin{array}{cc}
0 & I \\
-M^{-1} K & -M^{-1} D
\end{array}\right] \\
B=[0]
\end{gathered}
$$

In order to represent the effects of gravity and lift properly these matrices need to be modified to include the aforementioned. Previous work by Menon et. al. describes the derivation 
of a hybrid model which includes the lift component of the flat plate as it generates self-induced lift as a result of its vertical flapping motion. To account for externally induced lift when being exposed to a non-zero freestream velocity $u_{0}$ and angle of attack $\alpha$, lift was added as an additional state to expand the state space system. For this an elliptical lift distribution was assumed. Equation (5.44) is used to approximate the contribution of the externally induced lift.

$$
L=\left(C_{l_{0}}+2 \pi \alpha \frac{4}{\pi L 2}\right) \bar{q} S
$$

While this approximation of external lift forces in itself is very limited in terms of accuracy, it suffices for the purpose of contributing to the prediction for the wing deflection and is corrected largely by the measurement, which is introduced within the Kalman state estimator. Since the wing is exposed to gravity when oscillating normal to the earth's reference frame, gravity must be added. It was assumed that the effect of gravity is evenly distributed across the wings surface and each element.

$$
F=m_{e} g
$$

where $m_{e}$ is the mass of each element. As previously mentioned, the state transition matrix equation (5.42) corresponds to a free vibration. Any lift produced by the flat plate when in flight as well as gravity could be introduced on the left-hand side as an input. For computational performance considerations it was desired to keep the system input matrix empty and convert the system such that both gravity and lift are introduced in the system matrix A. This was done by defining both quantities as additional states. The resulting A matrix of the modified state space system equation (5.46) is conveniently formatted to fit into the predictor of the Kalman Filter Routine equation (3.15) -equation (3.19), where $D, K$ and $M$ of size $30 \times 30$, and $G$ and $L$ are of size $30 \times 1$. The state vector containing the displacement $w$ as well as the spacial derivatives and 
their respective time derivatives, gravity and lift is of size $62 \times 1$.

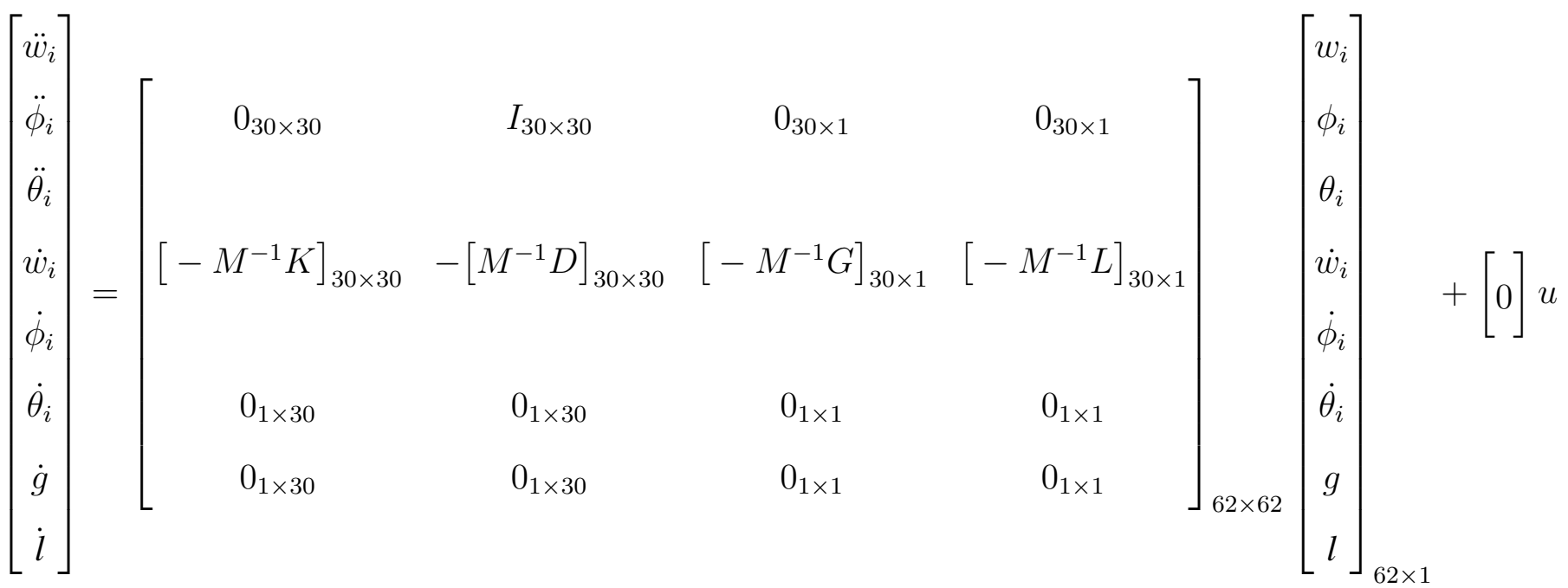

To complete the Kalman filter design complimentary measurements must be obtained. An inertial measurement unit (IMU) motion sensor with incorporated sensor fusion of accelerometer and gyroscopic data takes classical measurements at each node and supplies the data to the Kalman filter. For a simple 4 element flat plate model we use two span-wise rows of sensors, consisting of 4 sensors per row for a total of 8 sensors Figure 5.3.

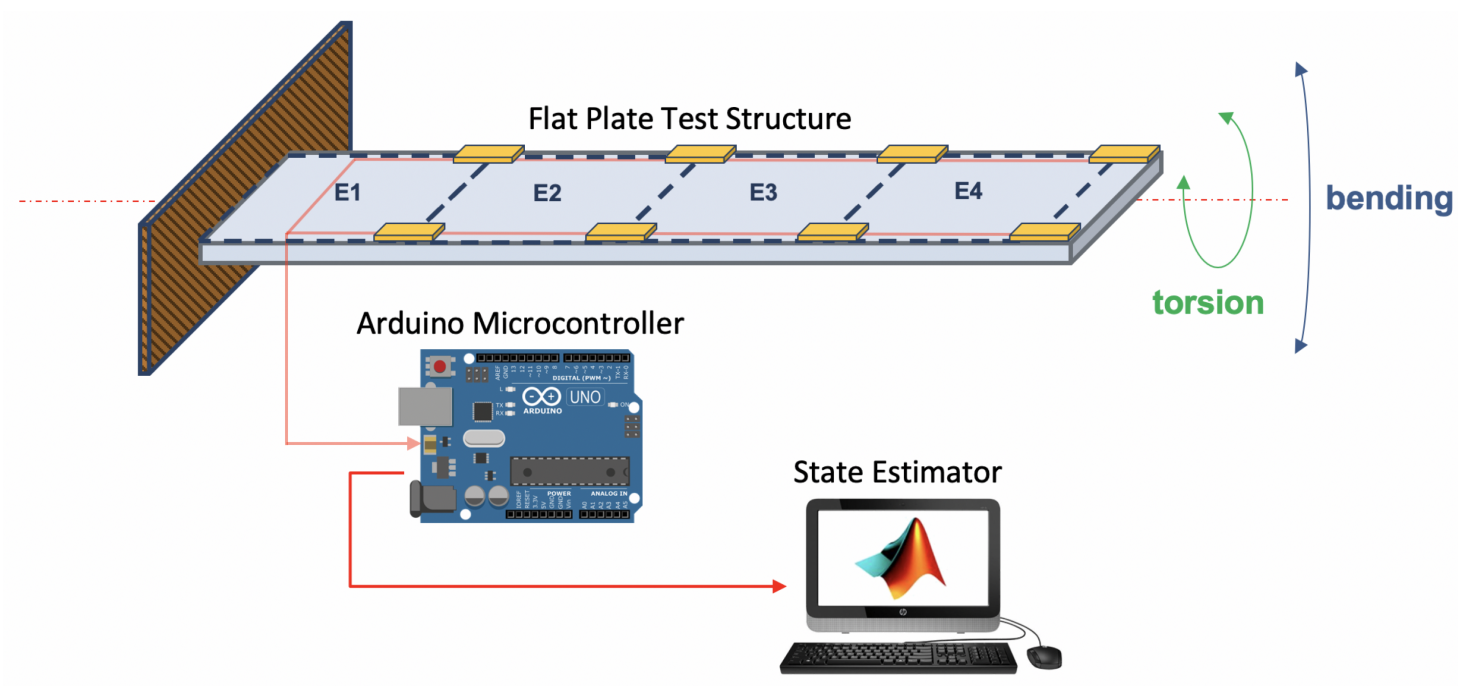

Figure 5.3: Illustration of sensors system arrangement

These sensors in combination with the associated known boundary conditions at the flat 
plate wing root make up four elements, each spanning across a $15 \mathrm{~cm}$ square. The structure used for the flat plate consist of a fiber glass printed circuit board. The board serves two purposes, namely the embedded circuitry, the exact sensor placement and the flexible yet durable nature of the circuit board material that is ideal for testing. Figure 5.4 and Figure 5.5 show the sensors placed on a printed circuit board.

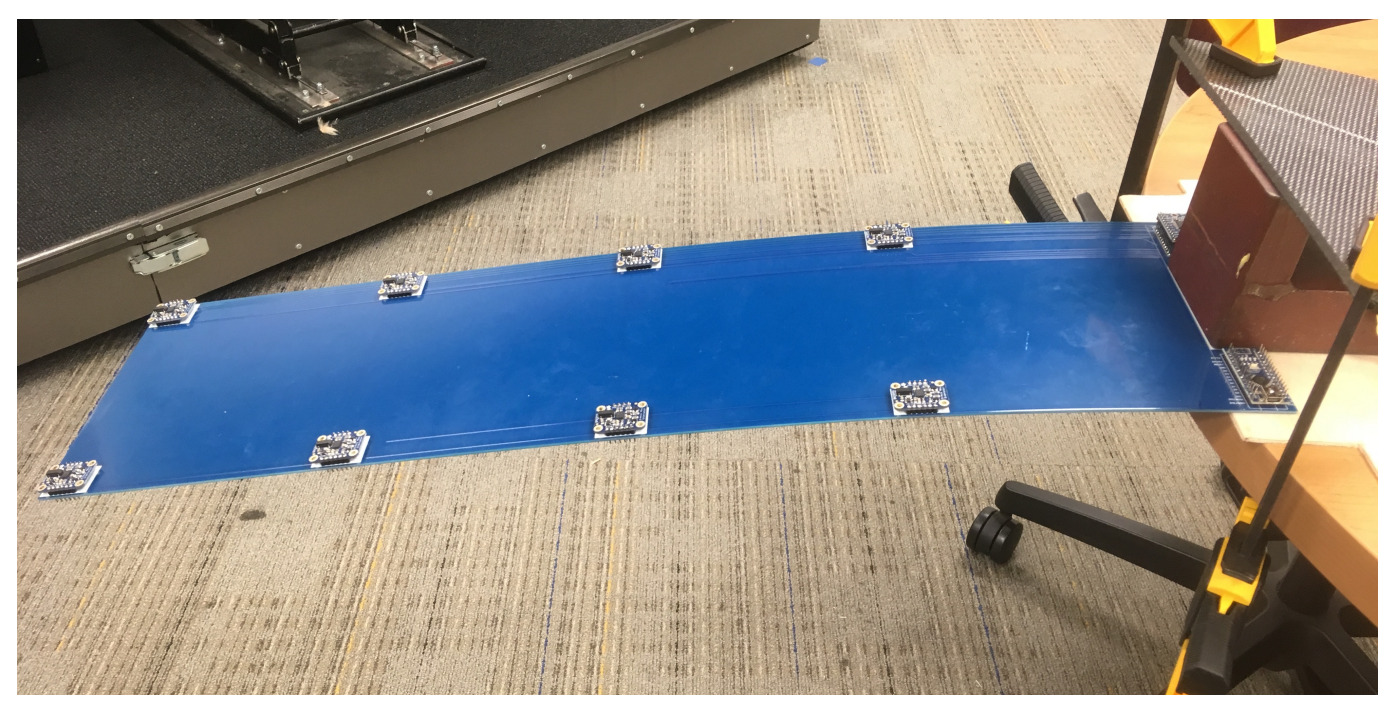

Figure 5.4: Sensor Arrangement

The exact machining of the circuit board allowed for precise sensor placement as shown in closeup of Figure 5.5

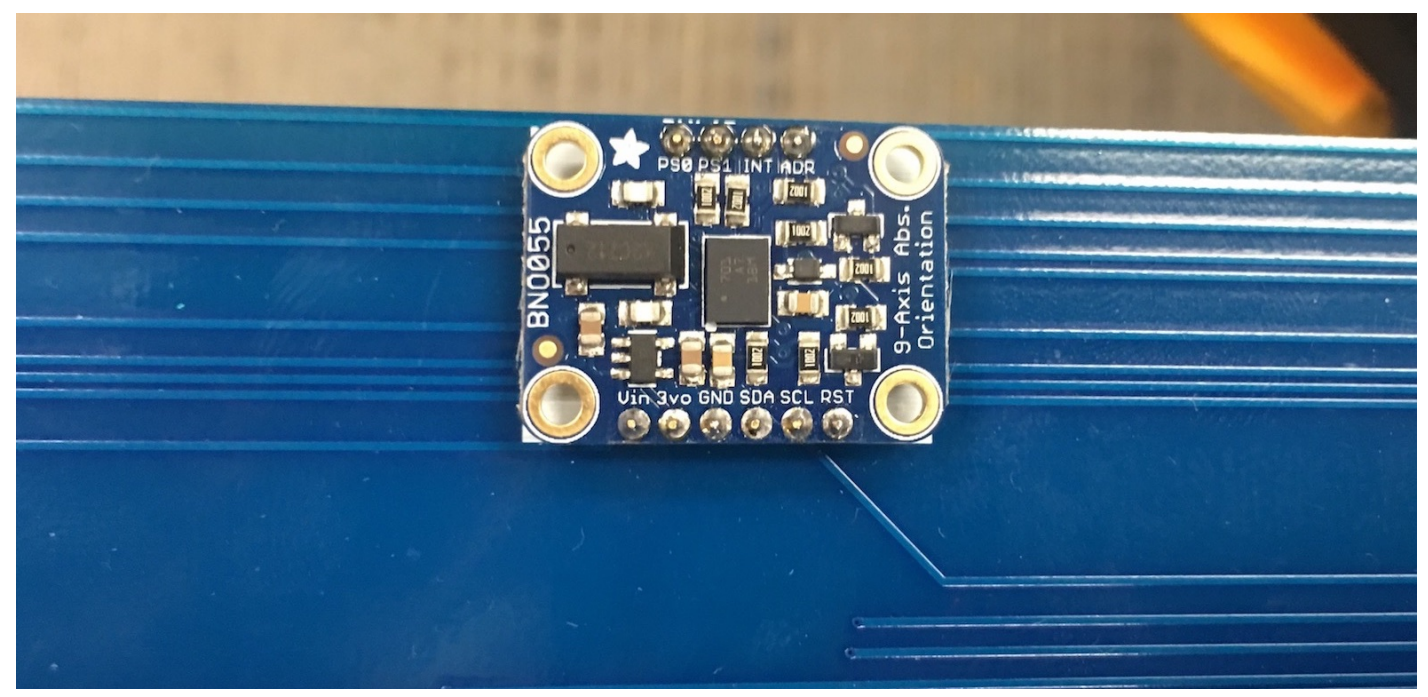

Figure 5.5: Close up view of sensor integration 
Section 5.2.1 shows the associated cost with the system setup described in this paper.

TABLE 5.1

\section{COST FOR SYSTEM SETUP}

\begin{tabular}{lr}
\hline Item & Cost \\
\hline BNO055 IMU Motion Sensor (8 total) & $\$ 31.93$ ea \\
PCB print (per board) & $\$ 16.41$ \\
Arduino Uno Micro controller & $\$ 18.90$ \\
Adafruit TCA9548A Multiplexer & $\$ 9.62$ \\
\hline TOTAL & $\$ \mathbf{2 9 7 . 3 7}$
\end{tabular}

Structural parameters such as Young's modulus and Poison's ratio for the PCB were initially taken as reference values from the work of Bhavsar et. al.[35]. Parameters were experimentally modified to assure realistic and matching unforced response characteristics of the model, to perform like the actual flat plate Section 5.2.2. For this, the circuit board was clamped and exposed to a deflection and subsequently allowed to oscillate freely. The experimental setup is shown in Figure 5.6. The flat plate wing structure is rotated $90^{\circ}$ and clamped at the end to remove the effect of gravity on the oscillation. Gravity will be added to the state space model later on. Note that the effect of aerodynamic forces are assumed to be negligible for the purpose of tuning the material properties. 


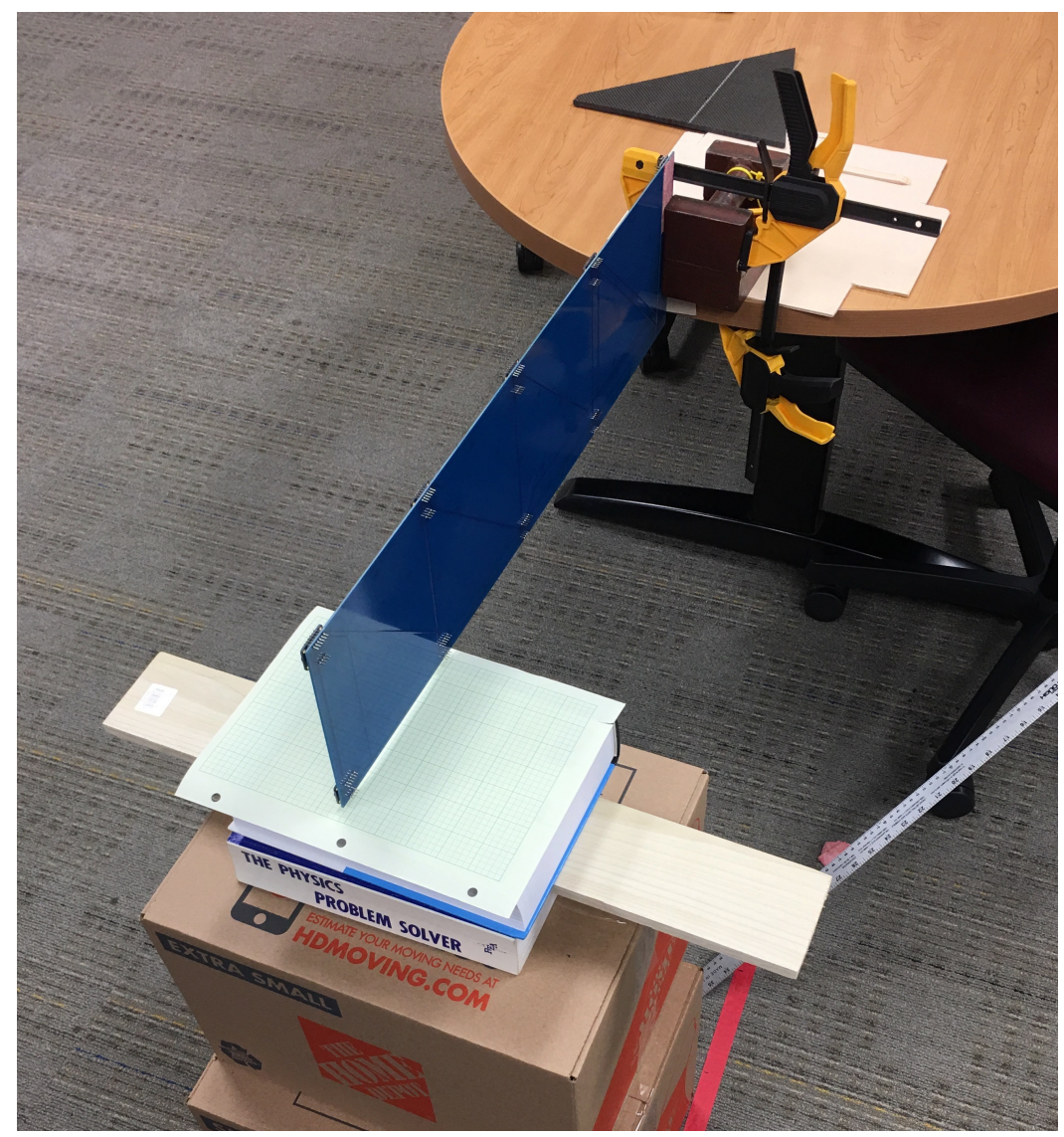

Figure 5.6: Experiment to obtain tune material properties

The unforced response of the PCB was captured in slow motion using a video recording device. The resulting deflection and oscillatory characteristics were visually obtained by inspection of the recorded overhead camera footage. Based on the performance of the flat plate, the material properties of the model were modified as described below so that the prediction matches the dynamic behavior of the flat plate. The governing dynamic equation of a flat plate described is given by Figure 3.3. The equation corresponds to a free vibration, any lift produced by the flat plate when in flight can be introduced on the left hand side.

\subsubsection{Measurement}

In order to utilize a Kalman filter algorithm, a measurement of the states shown in equation (5.46) must be available to complement and correct the prediction. The measurement of $w$ at the nodal locations is implicitly obtained from the BNO055 sensor array Figure 5.4 and Figure 5.5. Explicit measurements for the angle states $\phi$ and $\theta$ can be acquired directly from the 
sensor. The displacement $w$ must be approximated step-wise by using the trigonometric relationship between the span wise nodes and the angles measured at preceding nodes. An Euler transformation is used to transform the element wise deflection into the global coordinate system. Intermediate locations can be approximated using a spline fit.

TABLE 5.2

MATERIAL PROPERTIES

\begin{tabular}{lrr}
\hline Material Property & Reference Value & Modified Value \\
\hline Young's Modulus E & $26.12 \mathrm{GPa}$ & $33.04 \mathrm{GPa}$ \\
Poisson's Ratio $\nu$ & .139 & 0.39
\end{tabular}

\subsubsection{Kalman Filter applied to FEM prediction}

Similar to the kinematic approach, components of the Kalman filter were assembled. A Gaussian sensor error distribution was assumed and the needed standard deviation of the IMU motion processor was obtained by collecting an array of sensor readings at rest for the measurement. Since the covariance matrices for process and measurements are of large format due to the number of elements, nodes and degrees of freedom, these matrices are not shown in this paper. The error covariance matrix $P_{0}$ was set to be an identity matrix. Analogously to kinematic approach we followed the Kalman routine as shown in equations (3.15) to (3.19) with the exception that the state prediction $x^{-}$is performed in real time by a numerical solver in Matlab ${ }^{\circledR}$ and then placed into the Kalman routine. Figure 5.7 shows the principle of pulling data from the sensors and combining the information with data from a prediction. An elaborate and sophisticated experimental setup was developed to perform high accuracy testing and validation. The testing was conducted using calibrated equipment to capture the actual deflection and torsion and comparing true deflection to the computed state estimates. The next section will discuss the equipment as well as the evaluation process in detail. 


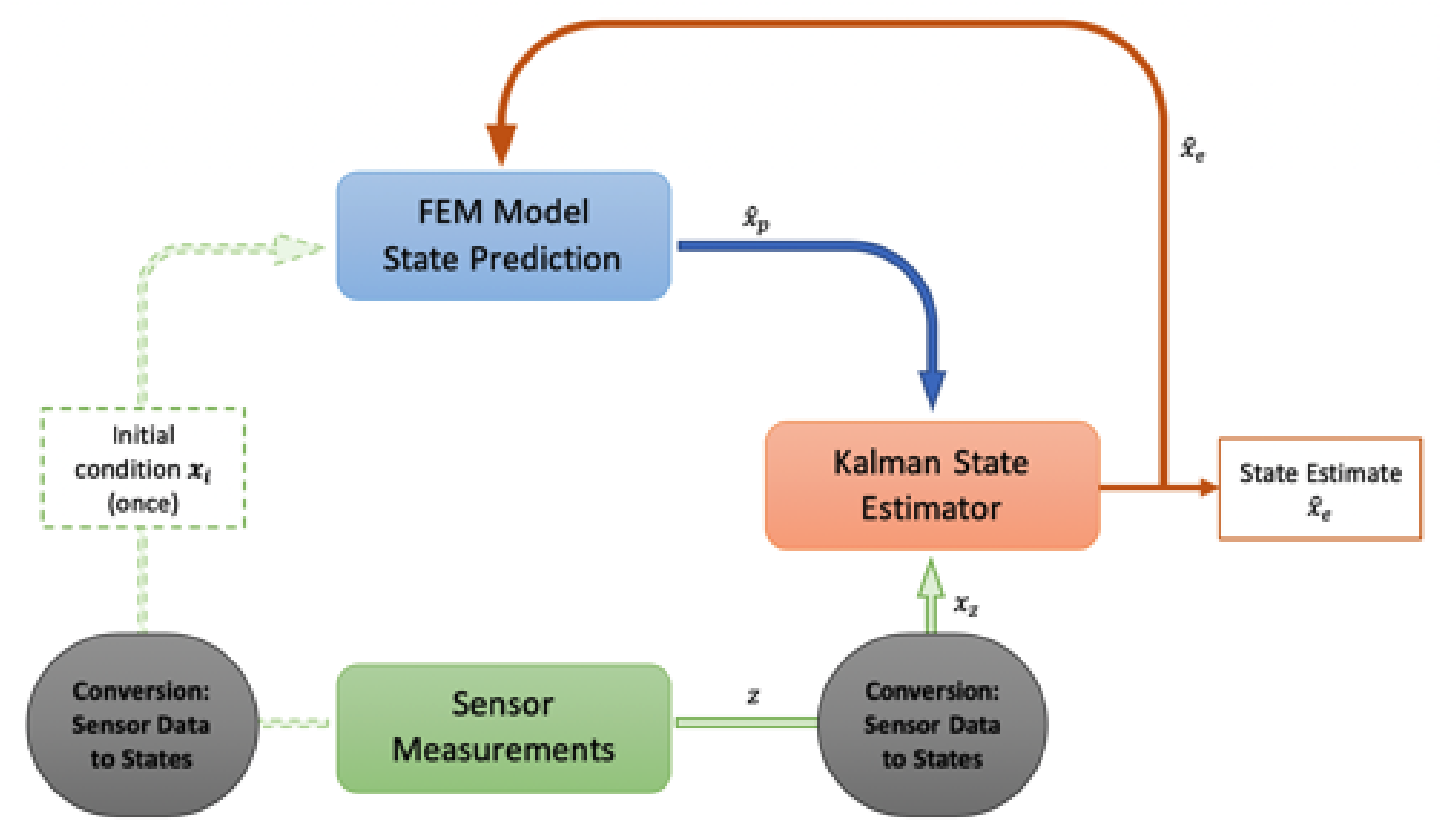

Figure 5.7: Kalman State Estimation Flow Chart

\subsection{Experimental Testing}

\subsubsection{Structural Dynamic Bench Testing}

First, precision static and dynamic bending and torsion tests were performed in collaboration with the WSU Flight Structures Laboratory . These experiments provided a detailed analysis of the system performance with various types of deformations and system configurations. The method used to obtain the actual deflection of the flat plate was an optical method similar to the one described by Veerman et. al. [15]. A speckle pattern was applied to the flat plate test section. Figure 5.8 shows the flat plate on the right with the applied speckle pattern. The airfoil section on the left was used later on in the wind tunnel. 


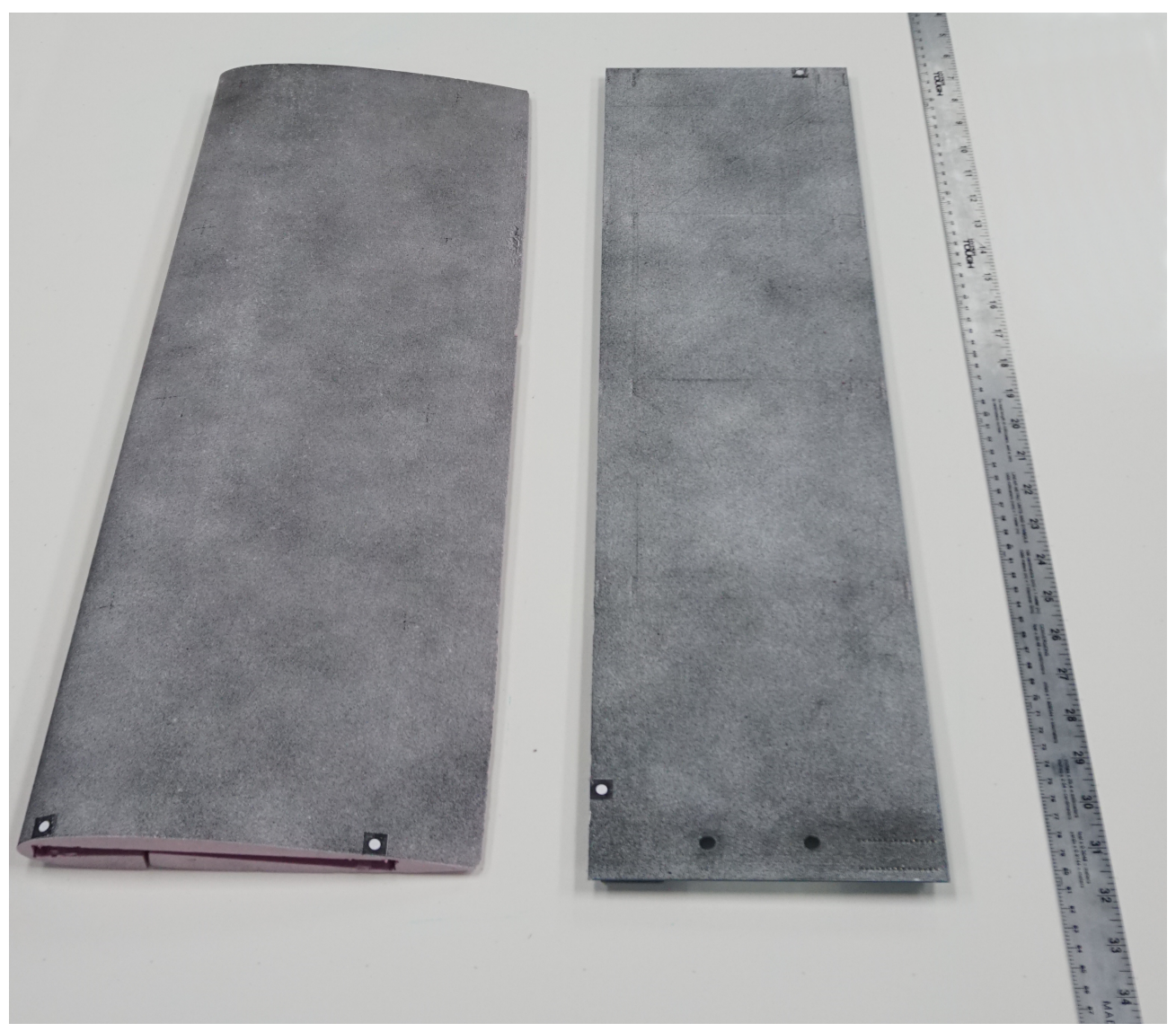

Figure 5.8: Foam Airfoil Enclosure and Flat Plate

Advanced camera equipment and image processing software was used to compute the vertical deflection of predetermined nodal points which coincide with the sensor locations. The software correlates changes in the appearance of the speckle pattern to vertical displacement. Results were then compared to the Kalman filter state estimates and used to progressively modify the system parameters as more test runs commenced in an effort to optimize the sensor system performance. Figure 5.9 shows the flat plate wing structure and mounting bracket that was used to clamp the wing root. 


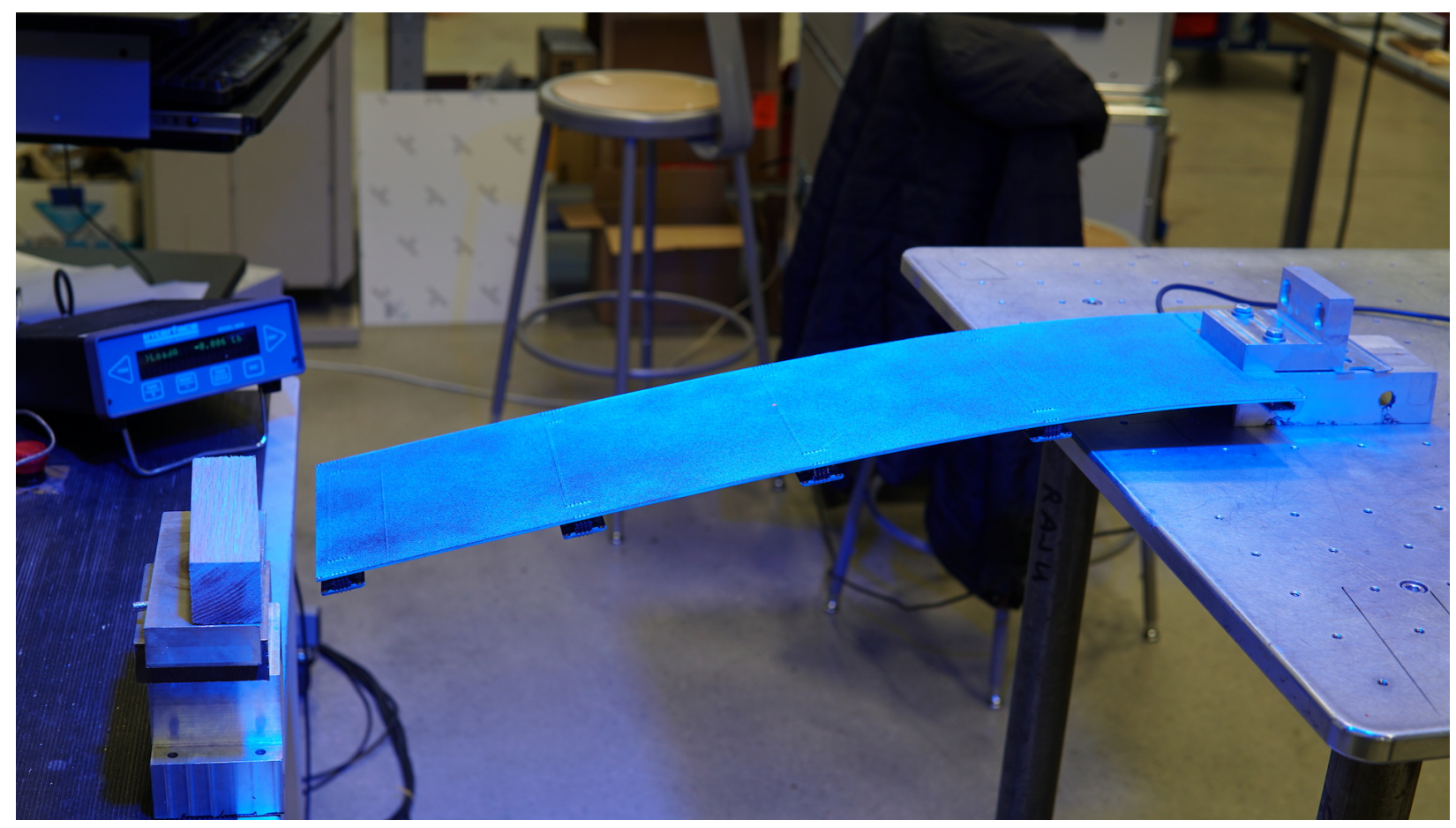

Figure 5.9: Experimental Setup

Figure 5.10 shows the dual camera setup used to capture the deformations by computing and correlating variances in the the speckle pattern.

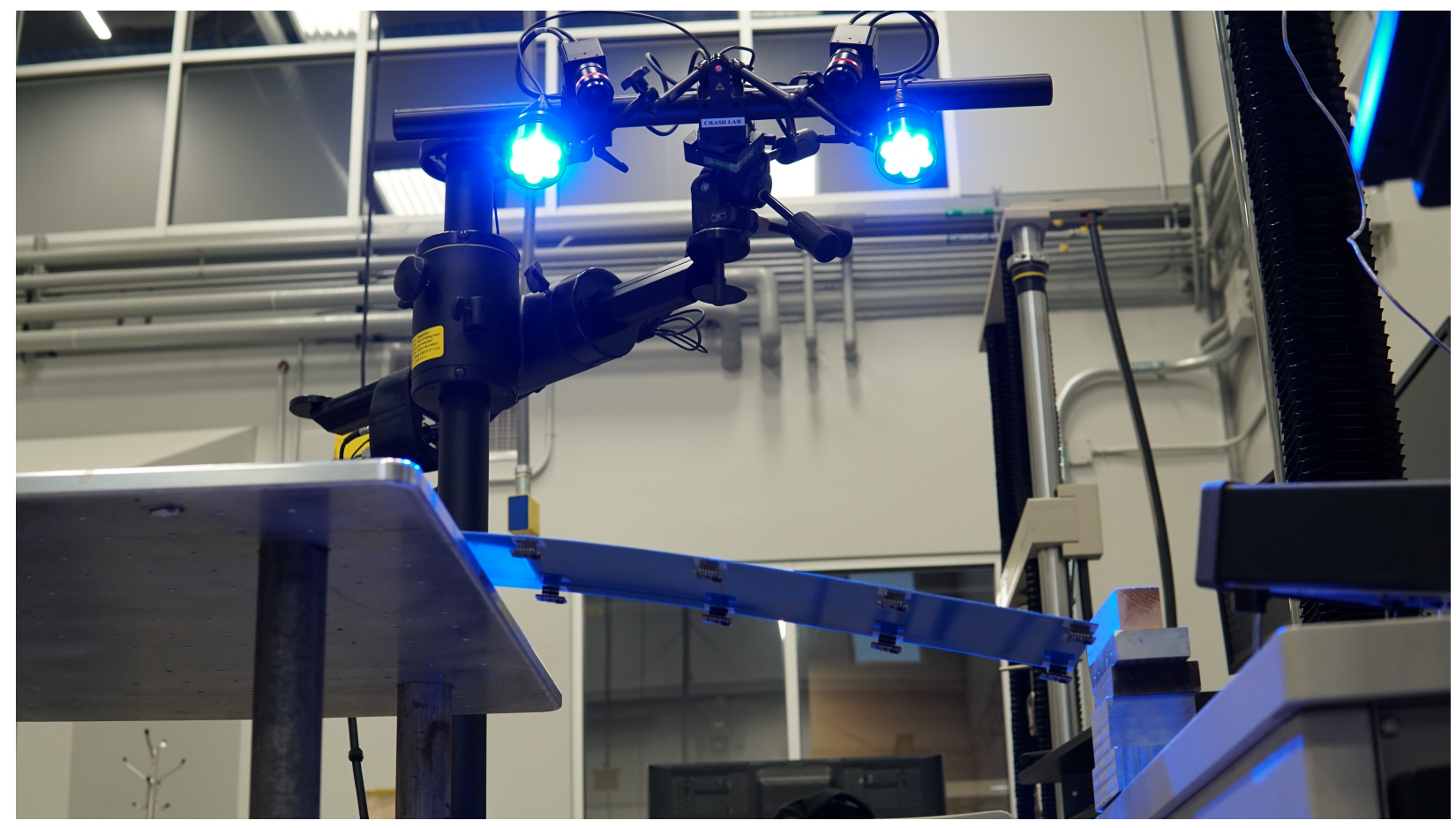

Figure 5.10: Experimental Setup 


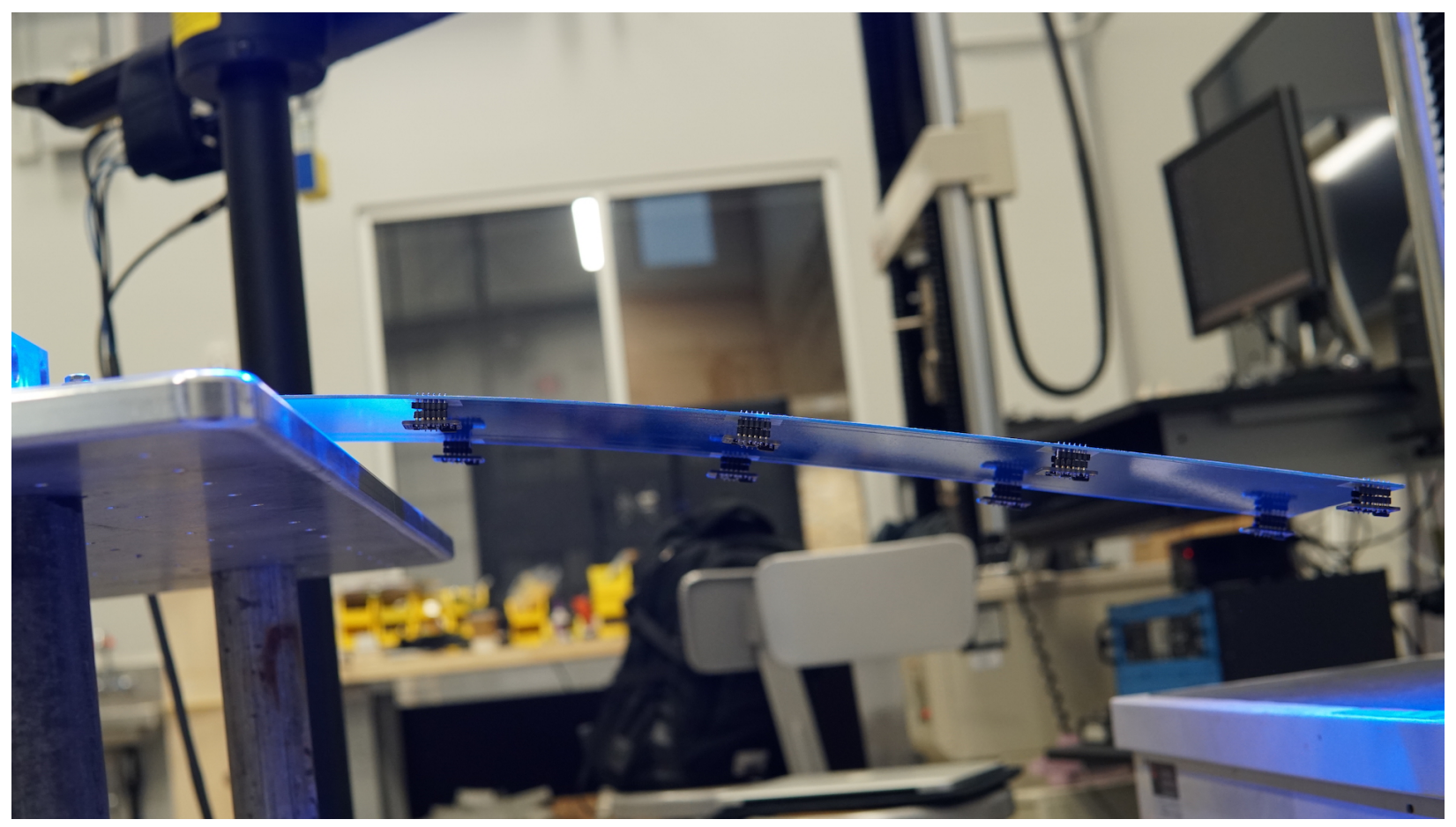

Figure 5.11: Experimental Setup

The tests conducted were free vibration test with varying initial conditions of the clamped flat plate. Theses initial conditions included a 1st order bending of the flat plate with no intentional twist, a sinusoidal s-shaped bending of the flat plate, and a 1st order bending coupled with a first order twist. Figure 6.2 and Figure 6.8 illustrate the nature of the initial deformation. The flat plate structure was deformed according the aforementioned conditions and subsequently released at which point the Kalman filter state estimator was initiated. The flat plate motion was captured using the optical validation equipment and compared to the Kalman filter state estimation. The results are discussed in chapter 6 .

\section{$5.4 \quad$ Wind Tunnel Testing}

Wind tunnel tests using a the flat plate with and without a foam airfoil enclosure Figure 5.8 were conducted with the intent of simulating realistic flight conditions. Some of the challenges that were faced included finding a technique to adequately validate the Kalman state estimate without introducing additional error and uncertainty. Furthermore building a wind tunnel mount that would sufficiently support the wing structure without jeopardizing integrity of the wire traces on the PCB 
took some effort. The details of experimental setup and process are described in this section.

\subsubsection{3x4 Wind Tunnel}

Initial experiments we setup in the $3 \times 4$ wind tunnel using a full wing. It was assumed that the existing Matlab ${ }^{\circledR}$ code could easily be expanded to incorporate a full wing section Figure 5.12

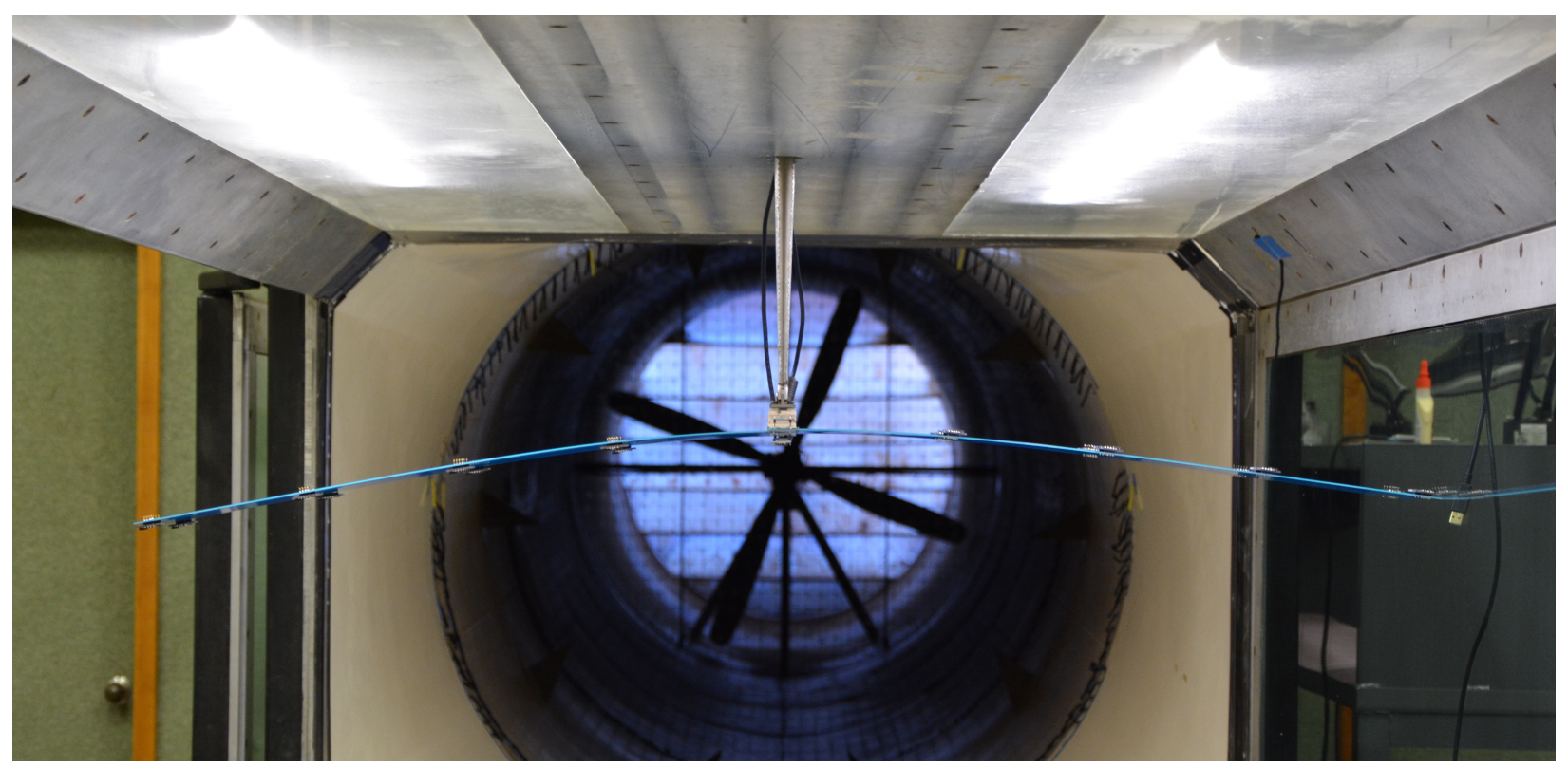

Figure 5.12: Full Wing in WSU $3 \times 4$ Wind Tunnel

However, in the wind tunnel it became apparent that doing so would create a dependence between the two half-wing section and led to undesired harmonic oscillations due to the non-rigid mount. Furthermore a significant adverse drift was discovered. Initially the source of the drift was not easily identifiable. Possible causes included temperature fluctuations, high degree vortex shedding or micro turbulence in the airflow. To conserve resources it was desired to determine the cause for the drift. An unorthodox experimental approach was taken to narrow down possible contributions. The double wing section was mounted on the back of an open vehicle and exposed to a free air stream as shown in Figure 5.13. 


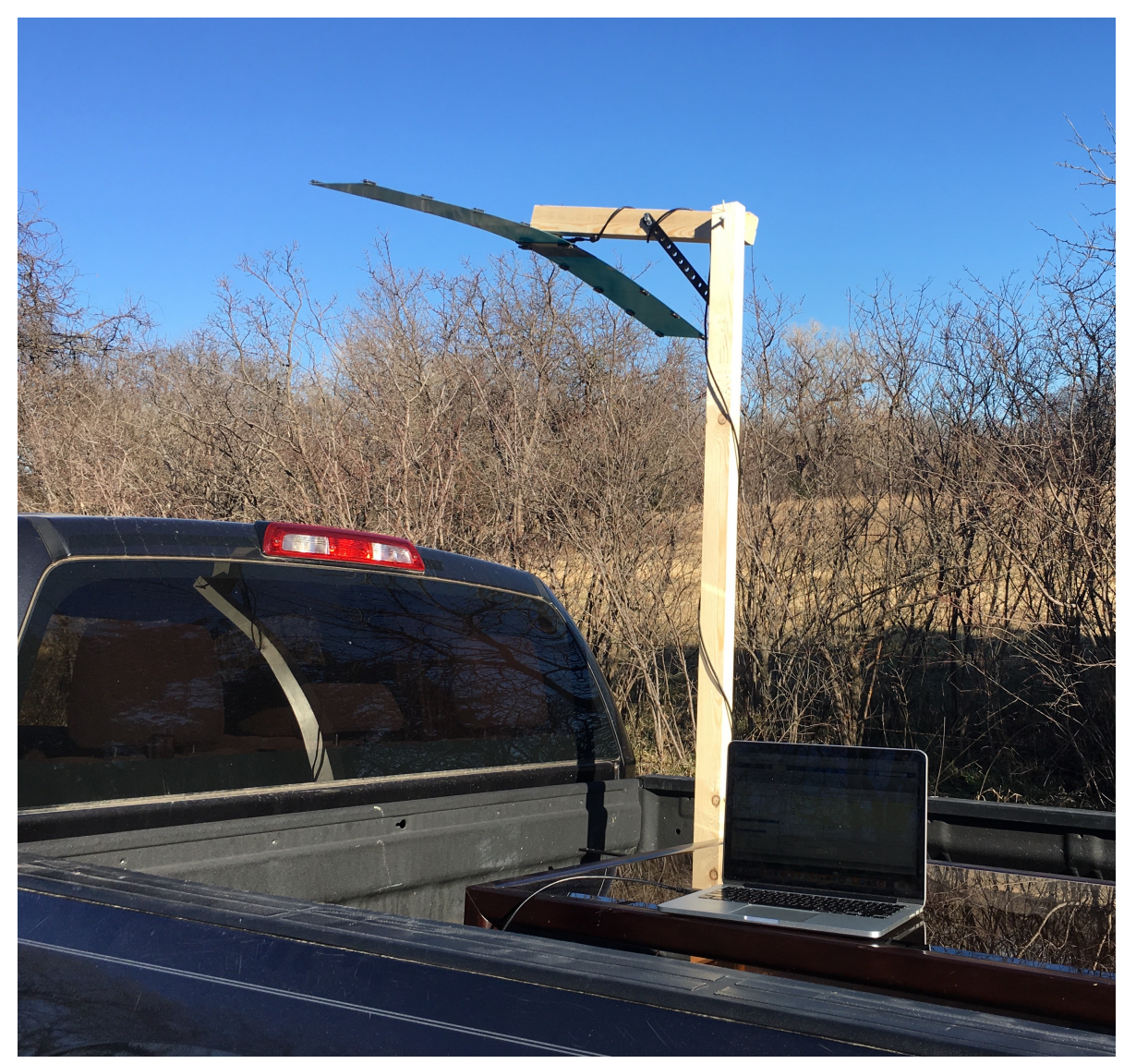

Figure 5.13: Full wing mounted on vehicle for testing

The results of this test showed significantly less sensor drift and also indicated that the sensor were capable of sensing even higher frequency vibrations like those encountered during the test. It was determined that the $3 \times 4$ wind tunnel exhibited native vibrations, likely due to the electric propeller system which can be seen in the background of Figure 5.12 as well as the close proximity of the wing tip to the tunnel wall. Further testing was therefore moved to the WSU Walter Beach Wind Tunnel.

\subsubsection{WSU Walter Beech Wind Tunnel}

The experimental setup was then limited to a half wing section and moved to the WSU Walter Beech 7x10 wind tunnel. In order to provide optimal result validation the 3-D high precision camera equipment from the WSU Flight Structures Laboratory was installed on top of the 7x10 
wind tunnel Figure 5.14 Test runs were conducted at $2.5 \mathrm{PSF}$ (approximately $45 \mathrm{ft} / \mathrm{s}$ ) and increasing angles off attack from -5 to +20 degrees.

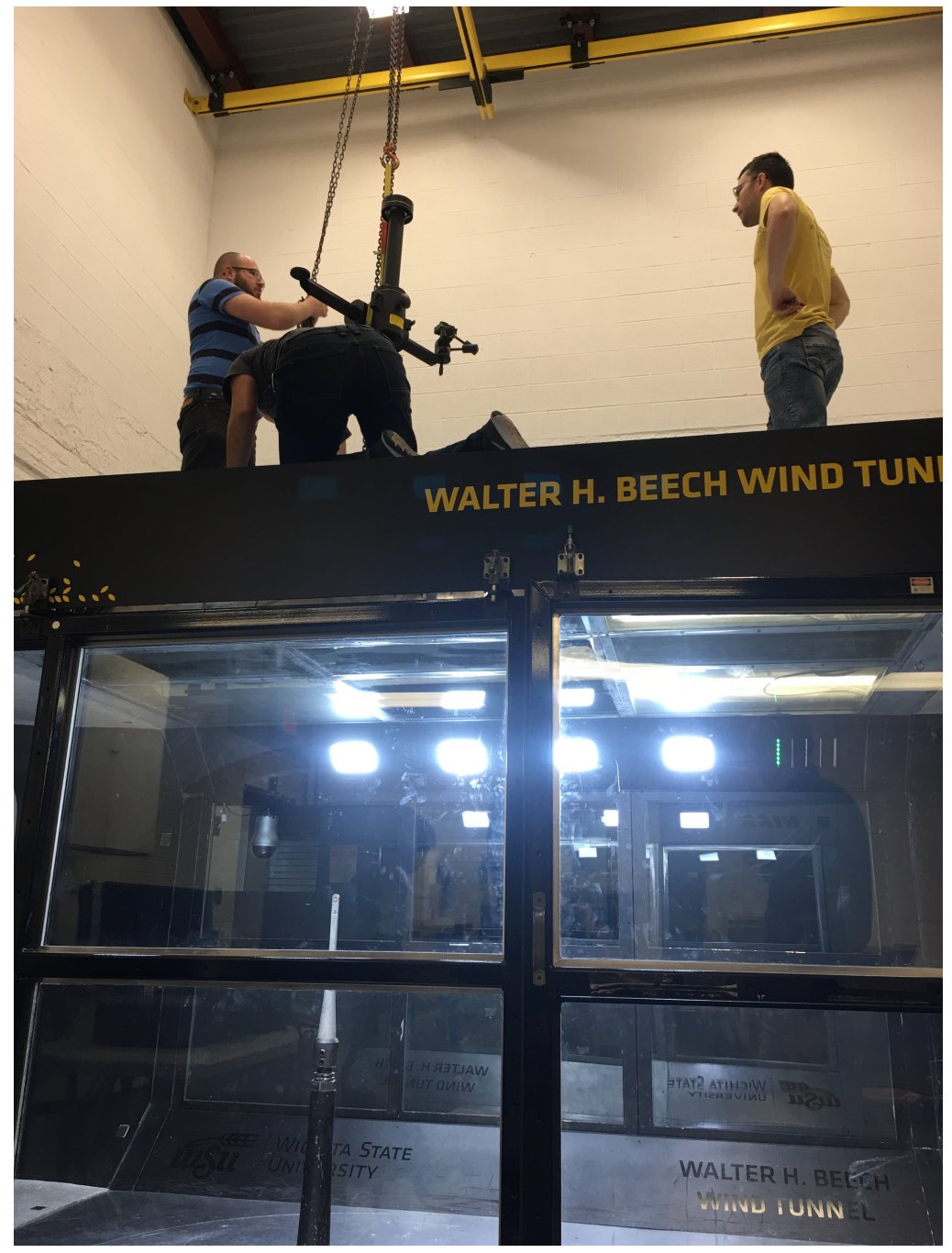

Figure 5.14: Optical Measurement System Installation

Figure 5.15 shows the original flat plate PCB wing structure installation in the 7x10 wind tunnel. The camera setup is located above the wing and captured the wings deflection through the glass ceiling. 


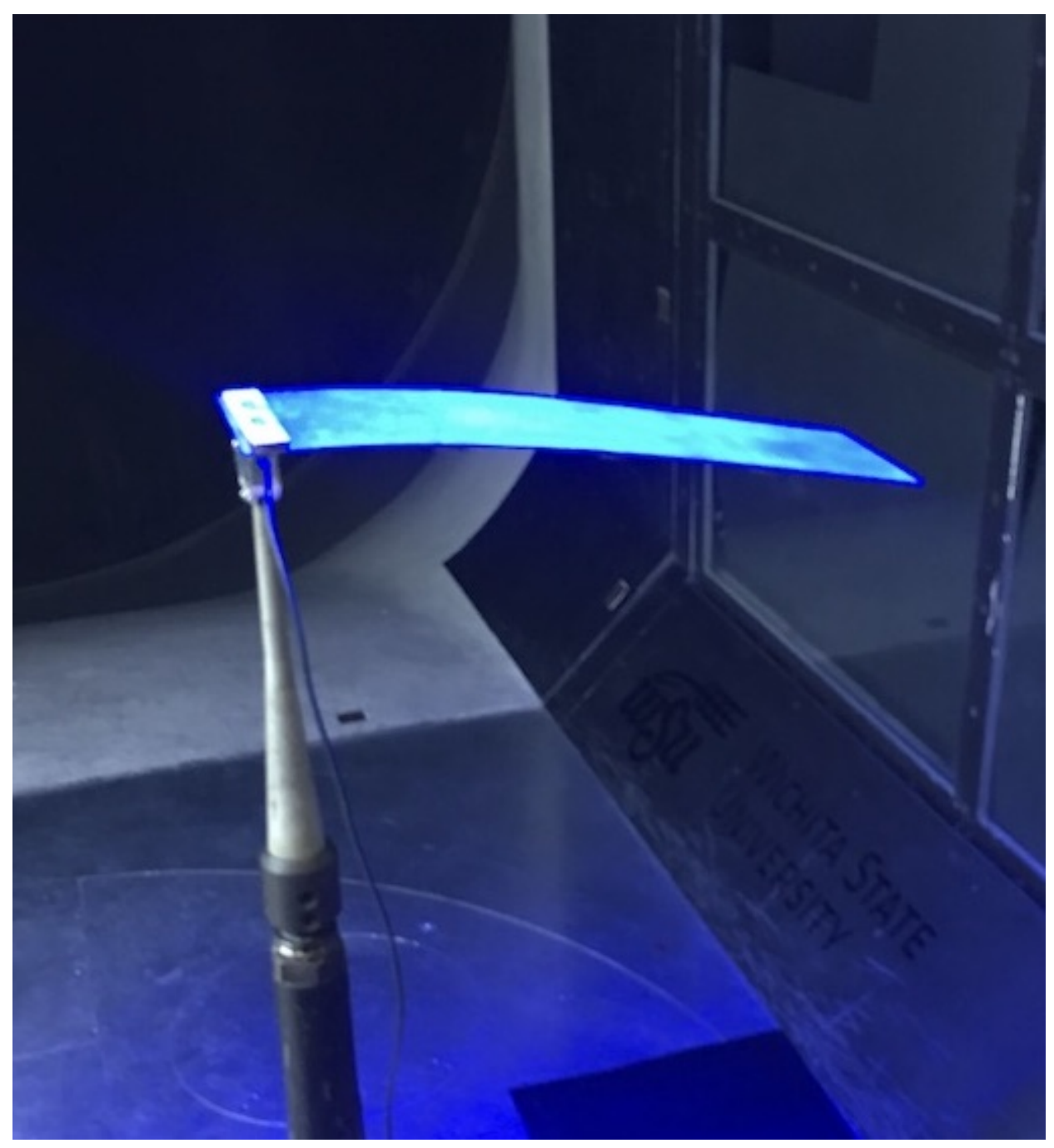

Figure 5.15: Half wing section PCB mounted in 7x10 wind tunnel

Because of the recurring vibration due to the flutter induced by the stall around the sensors on the lower surface, much of the experimental testing in the wind tunnel included the installation of the foam airfoil. Figure 5.16 shows the modified wind tunnel mount with the half wing section PCB inside of a foam airfoil enclosure. 


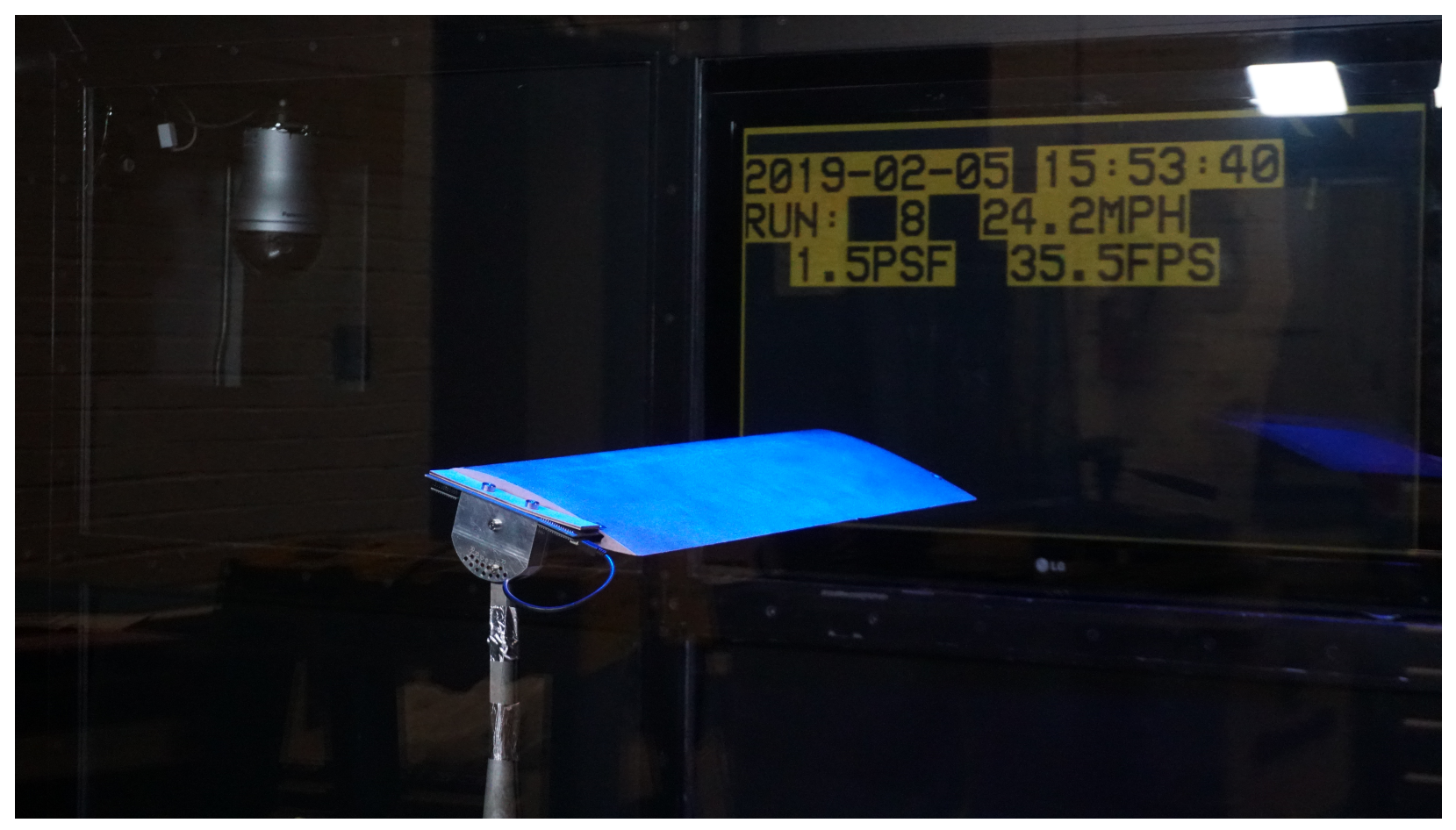

Figure 5.16: Half wing section PCB with foam structure mounted 7x10 wind tunnel

Because the camera equipment had to periodically be re-calibrated between each run, every test run was very time consuming. Some of the data captured by the camera equipment was lost at high angles of attack when the camera lost visual track of the reference patter. Results from the WSU Flight Structures Laboratory and the Walter Beech wind tunnel are discussed in the following chapter. 


\section{CHAPTER 6}

\section{RESULTS AND DISCUSSION - PART 2: FINITE ELEMENT APPROACH}

Results discussed in this chapter are grouped and presented based on the respective experimental setup and environment as well as test condition and the corresponding initial conditions which were discussed in Chapter 5. Each subplot reflects the data for the the given node and sensor location as illustrated in the previously and in accordance with Figure 6.1. The graphs shown in this chapter represent the deflection of each sensor and the respective finite element node, based on prediction, measurement, estimate and actual deflection.

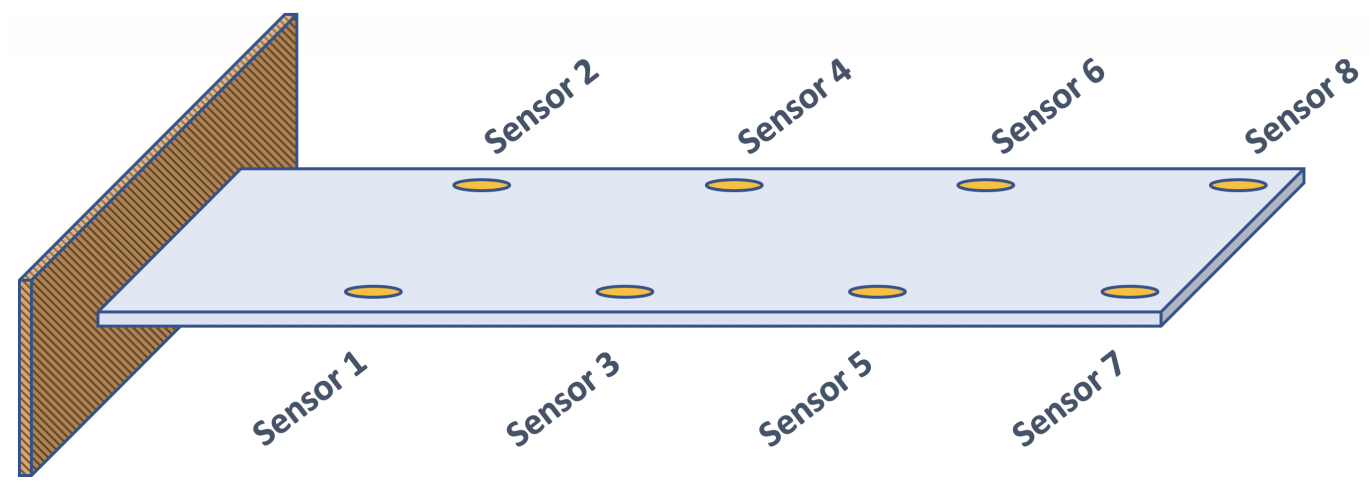

Figure 6.1: Sensor Layout and Numbering

\subsection{Structures Lab: Dynamic Response - Results}

\subsubsection{Free Vibration, Non-Zero, Bending, No Twist Initial Condition}

Figures 6.3 and 6.4 show the deflection data for the free vibration bending experiment with non-zero initial conditions, which was conducted in the WSU structural lab. The corresponding non-zero initial bending conditions are illustrated in Figure 6.2. Results from this experiment were among the first data sets which were validated with the high-accuracy 3-D optical measuring system. The structural model and its' material properties were those determined during the pretesting and calibration which was discussed in the previous Chapter 5 , Figure 6.3 corresponds to the leading edge nodes (sensors 1, 3, 5, 7) and Figure 6.4 corresponds to the trailing edge nodes (sensors $2,3,6,8$ ). Based on obtained preliminary results, the data for the state estimate was 
calibrated with a $0.01 \mathrm{~m}$ constant calibration offset for both leading and trailing edge.

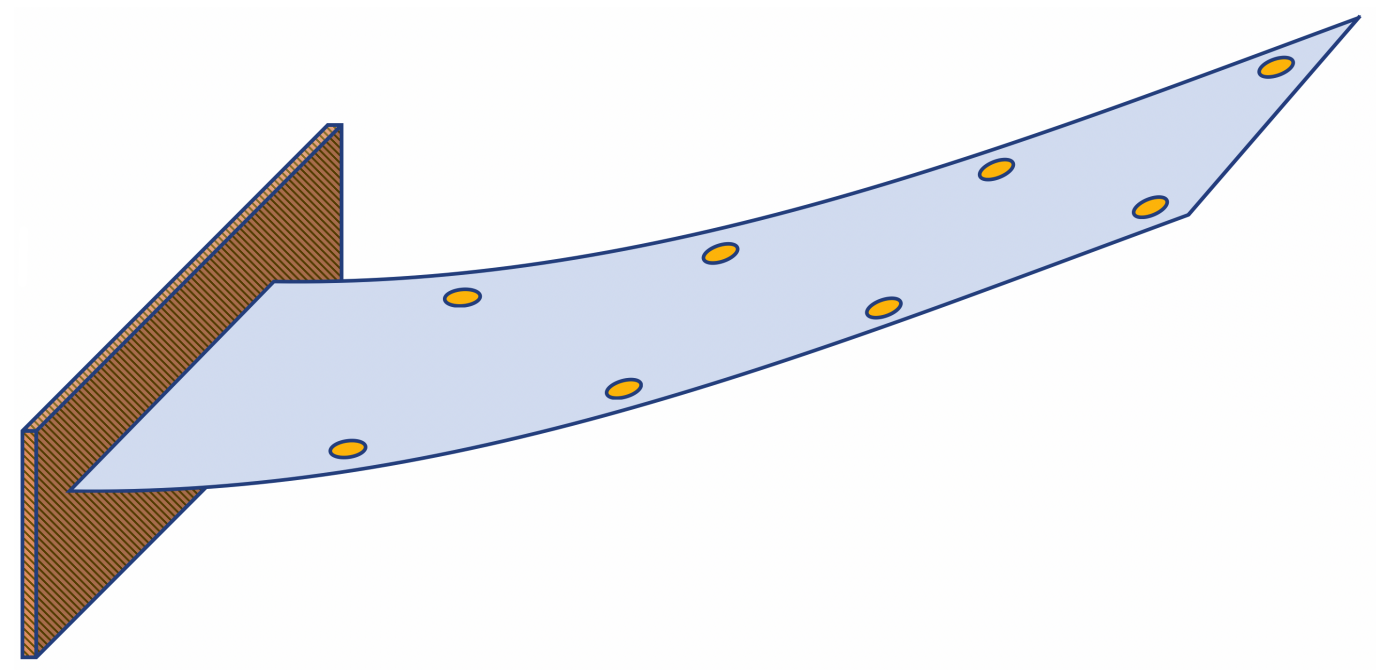

Figure 6.2: Free Vibration - Non Zero ICs

When inspecting the data, the initial non-zero conditions captured by the first measurement and actual deflection data match for all sensors. The peak steady state deflection is increasing from wing root to wing tip which is expected after the oscillation dampens out. During the time span between $t=0$ and $t=1$ seconds (time span enclosed by the blue box) all plotted outputs, namely prediction, measurement and estimate go through a transition period during which the state estimator covariance matrix converges and initial computation delays associated with initiating the Kalman filter are overcome. The following observations were made for both the leading edge and trailing edge sensor nodes: The transient amplitude of the actual deflection, captured by the optical test and validation equipment is slightly higher than the deflection of the state estimate. Analogously the measurement captured by the IMU sensors is of higher magnitude than the prediction and a maximum offset of approximately $5 \mathrm{~mm}$ is observed. This can likely be attributed to inaccuracies during the displacement approximation using sensor data and the Euler transformation. This error accumulates from from wing root to wing tip. The measurement tends to match the actual deflection more so than the prediction. From that two conclusions can be drawn: 1.) The finite element model required additional modifications mostly to the material properties. 2.) The Kalman filter covariance matrices needed adjustment to shift weight to the 
measurement. By inspection one can say that the settling time for prediction, measurement and estimate matches that of the actual deflection. Both prediction and measurement show little to no sign of process or sensor noise. The estimate tracks the actual deflection with an average accuracy of approximately $4 \mathrm{~mm}$ at the wing tip. There is small phase shift between all 4 curves: The prediction leads the actual deflection by approximately 1 time step of approximately 0.03 seconds. Inspection of the plots reveal a few differences in the graphs shown for the leading edge as opposed to the trailing edge. One isolated case of an offset in the state estimation of sensor node 1 can be observed for the leading edge. The root sensor of the leading edge shows a higher average offset between estimate and actual deflection of $5 \mathrm{~mm}$. In all graphs the blue prediction curve shows a slight positive offset when compared to the actual deflection. This is most noticeable for sensors nodes 3 and 4 of the leading edge Figure 6.3 .

Table 6.1 shows the root mean square error (RMSE) between the actual deflection as captured by the 3-D camera validation system and the state estimation as well as the measurement and prediction, both of which show a greater RMSE. The Kalman state estimate however shows a significantly lower root mean square error. This is desirable as it support the hypothesis that the Kalman filter successfully fuses the state prediction and the measurement into a 'best estimate' of what the actual deflection is.

TABLE 6.1

MEAN SQUARE ERROR FOR MEASUREMENT, PREDICTION AND ESTIMATE

\begin{tabular}{|c|c|c|c|}
\hline Sensor/Node & Measurement RMSE [m] & Prediction RMSE [m] & Estimate RMSE [m] \\
\hline Sensor 1 & 0.0018 & 0.0050 & 0.0060 \\
\hline Sensor 2 & 0.0049 & 0.0081 & 0.0047 \\
\hline Sensor 3 & 0.0083 & 0.0121 & 0.0048 \\
\hline Sensor 4 & 0.0054 & 0.0121 & 0.0052 \\
\hline Sensor 5 & 0.0125 & 0.0186 & 0.0092 \\
\hline Sensor 6 & 0.0096 & 0.0113 & 0.0094 \\
\hline Sensor 7 & 0.0176 & 0.0239 & 0.0137 \\
\hline Sensor 8 & 0.0168 & 0.0158 & 0.0140 \\
\hline Avg. RMSE & $\mathbf{0 . 0 0 9 6}$ & $\mathbf{0 . 0 1 2 9}$ & $\mathbf{0 . 0 0 8 4}$ \\
\hline
\end{tabular}



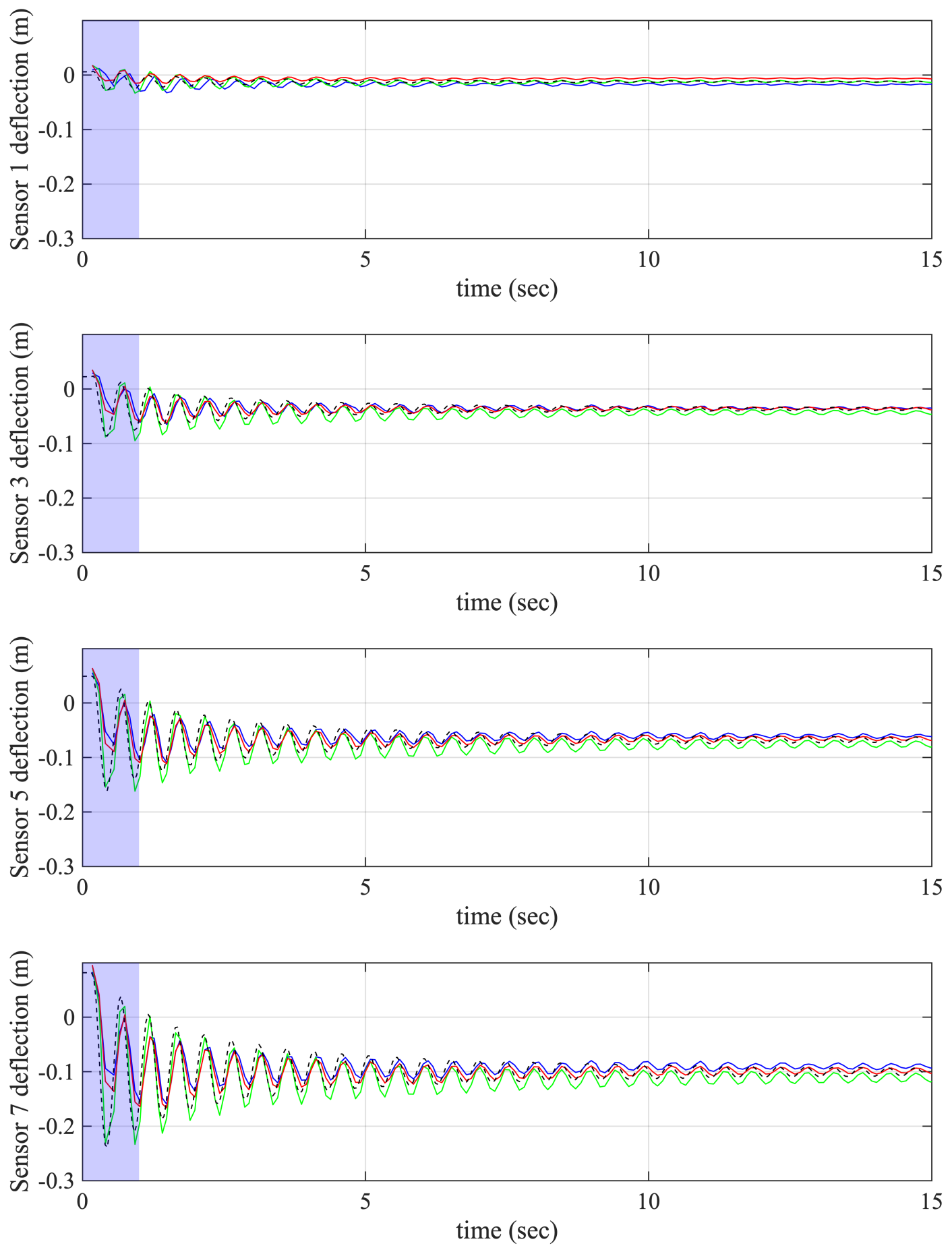

Model Prediction Kalman Filter Estimate

Sensor Measurement -....-.- Camera Measurement

Figure 6.3: Free Vibration: Non-Zero Bending Initial Conditions, No Twist, Leading Edge Sensor Row 

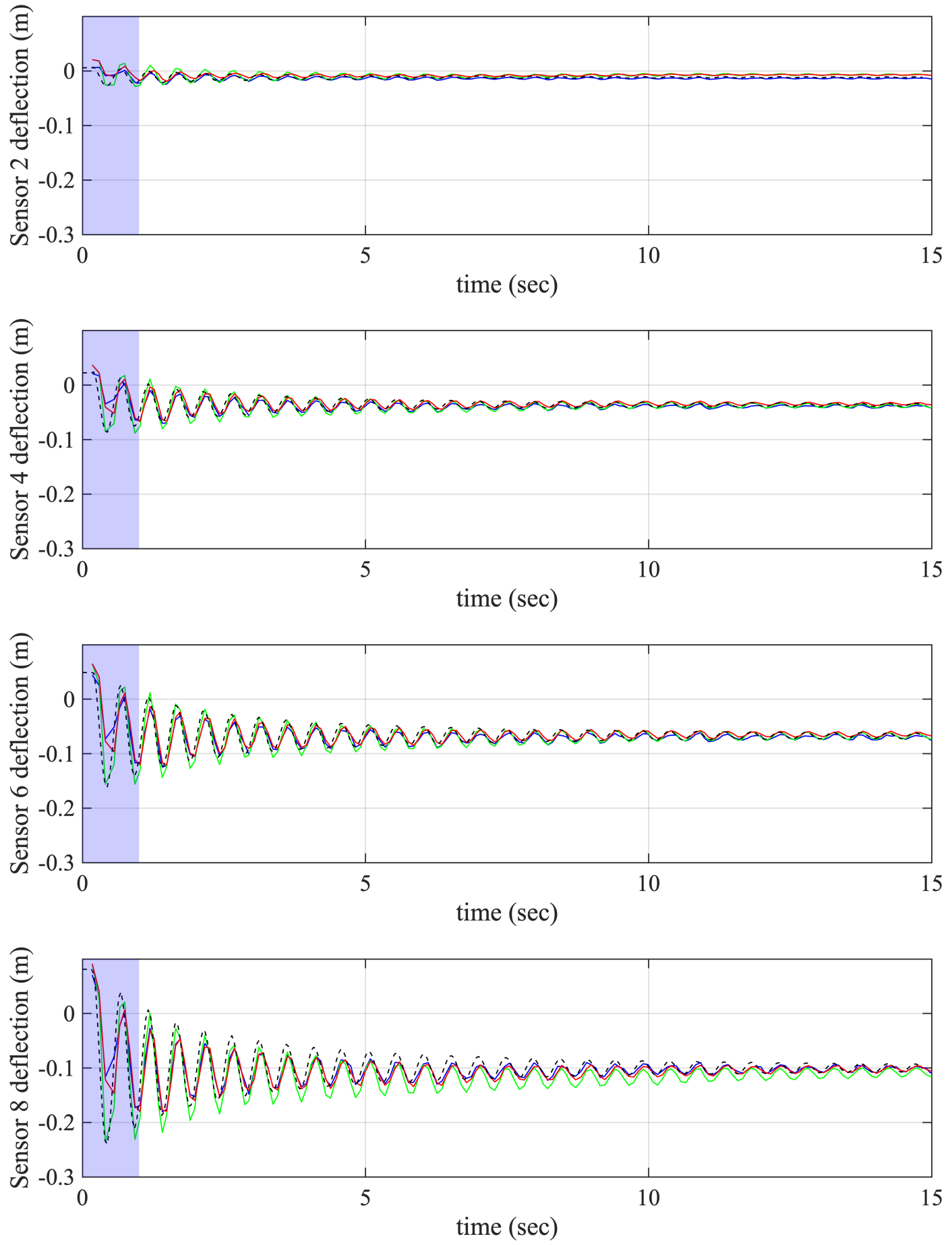

Model Prediction Kalman Filter Estimate

Sensor Measurement -....-.- Camera Measurement

Figure 6.4: Free Vibration: Non-Zero Bending Initial Conditions, No Twist, Trailing Edge Sensor Row 


\subsubsection{Free Vibration with complex S-shape Bending Initial condition}

Figures 6.6 and 6.7 correspond to the motion of the wing structure following the s-shape initial condition. Figure 6.5 illustrates the nature of the initial condition as pure bending without intentional twist. Note that the shown graphic is an exaggeration of the actual initial deformation. Due to length of the test section and the material characteristics which were bounding properties on the complexity of the initial deformation, it was not possible to bend the flat plate beyond 10 $\mathrm{cm}$ between highest and lowest point.

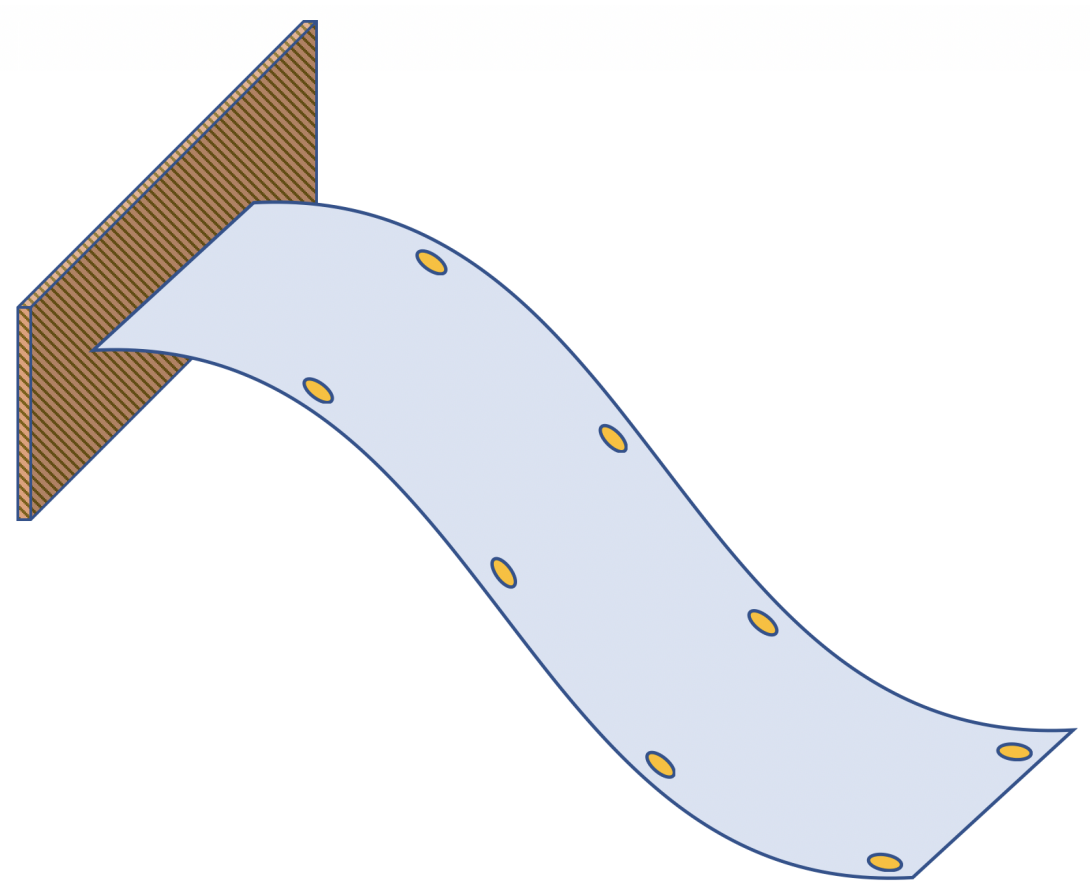

Figure 6.5: S-Shape Bending Initial Condition

The plots shows the initial conditions at $\mathrm{t}=0$ to be all positive with the exception of sensor nodes 5 and 6 which show a negative deflection. This deflection turns again positive towards the wing root sensor nodes. Note that the initial conditions as shown in the plot are not representative of the maximum deformations, which occurred at off-node locations. Data from all plots shows a near complete phase match along all sensors. Similar to the previously discussed data set for the simple bending case, the data cluster consisting of prediction, measurement and state estimate was calibrated in a post process with a $0.015 \mathrm{~m}$ constant calibration offset for both leading and 
trailing edge. During the time span $t=0$ and $t=1$ seconds all plotted data sets show erratic behavior which normalizes past $t=1$ second. In addition to the covariance convergence and the lag during the Kalman filter initiation, the validation data recording was started with an intentional delay. The sequence of launching the Kalman routine, data validation and test condition was found to be an integral consideration, which significantly effected the outcome of the results. During the first 5 seconds the oscillations of the measurement shows to be higher in magnitude than the actual deflection. This behavior vanishes and the peak-to-peak deflection of the measurement approaches similar values to those of the true deflection as both quantities approach the steady state deflection. Inspecting both, leading and trailing edge plots reveal a noticeably larger spread between prediction and measurement for the leading edge, particularly at the tip location. At the 3 second mark there appears to be a slight positive shift in prediction values. The measurement data suggests a consistently more negative deflection than the actual deformation. While this discrepancy between prediction and measurement is not desirable, the resulting estimate is still capable of tracking the actual deflection reasonably well with an maximum total error of $1 \mathrm{~cm}$ at the wing tip.

The root mean square error (RMSE) between the actual deflection the state estimation as well as the measurement and prediction is shown in Table 6.2. Similar to the the previous case, both prediction and measurement show a greater RMSE which further supports the benefit of a Kalman filter state estimator.

TABLE 6.2

RMS ERROR TABLE FOR S-SHAPE INITIAL CONDITION

\begin{tabular}{|c|c|c|c|}
\hline Sensor/Node & Measurement RMSE [m] & Prediction RMSE [m] & Estimate RMSE [m] \\
\hline Sensor 1 & 0.0039 & 0.0081 & 0.0098 \\
\hline Sensor 2 & 0.0074 & 0.0120 & 0.0074 \\
\hline Sensor 3 & 0.0072 & 0.0137 & 0.0048 \\
\hline Sensor 4 & 0.0055 & 0.0099 & 0.0049 \\
\hline Sensor 5 & 0.0138 & 0.0185 & 0.0080 \\
\hline Sensor 6 & 0.0103 & 0.0107 & 0.0083 \\
\hline Sensor 7 & 0.0168 & 0.0222 & 0.0117 \\
\hline Sensor 8 & 0.0173 & 0.0138 & 0.0128 \\
\hline Avg. RMSE & $\mathbf{0 . 0 1 0 3}$ & $\mathbf{0 . 0 1 3 6}$ & $\mathbf{0 . 0 0 8 5}$ \\
\hline
\end{tabular}



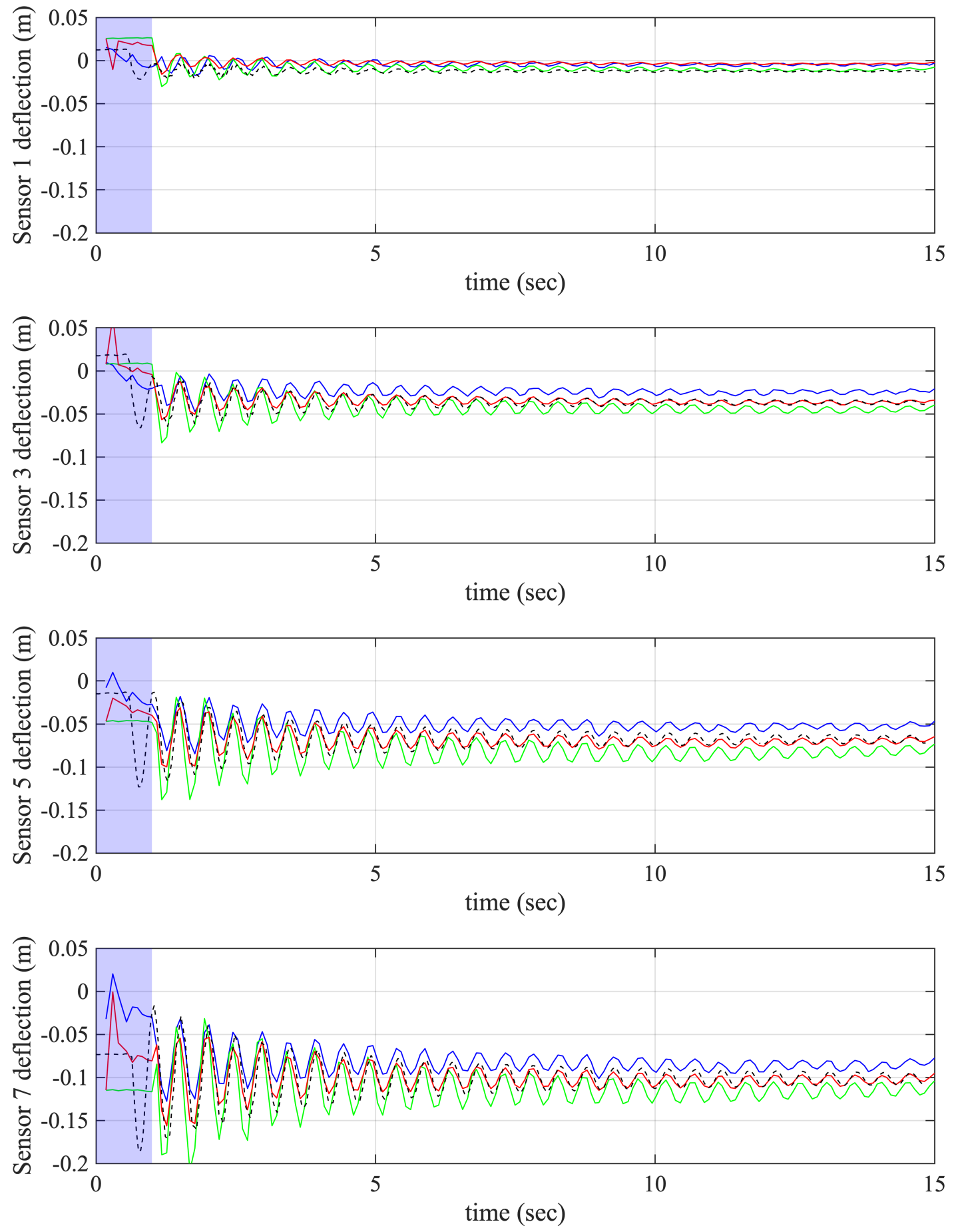

\begin{tabular}{|c|c|}
\hline $\begin{array}{l}\text { - Model Prediction } \\
\text { - Sensor Measurement }\end{array}$ & $\begin{array}{l}\text { Kalman Filter Estimate } \\
\end{array}$ \\
\hline
\end{tabular}

Figure 6.6: Initial Conditions: S-Shape Bending, No Twist, Leading Edge Sensor Row 

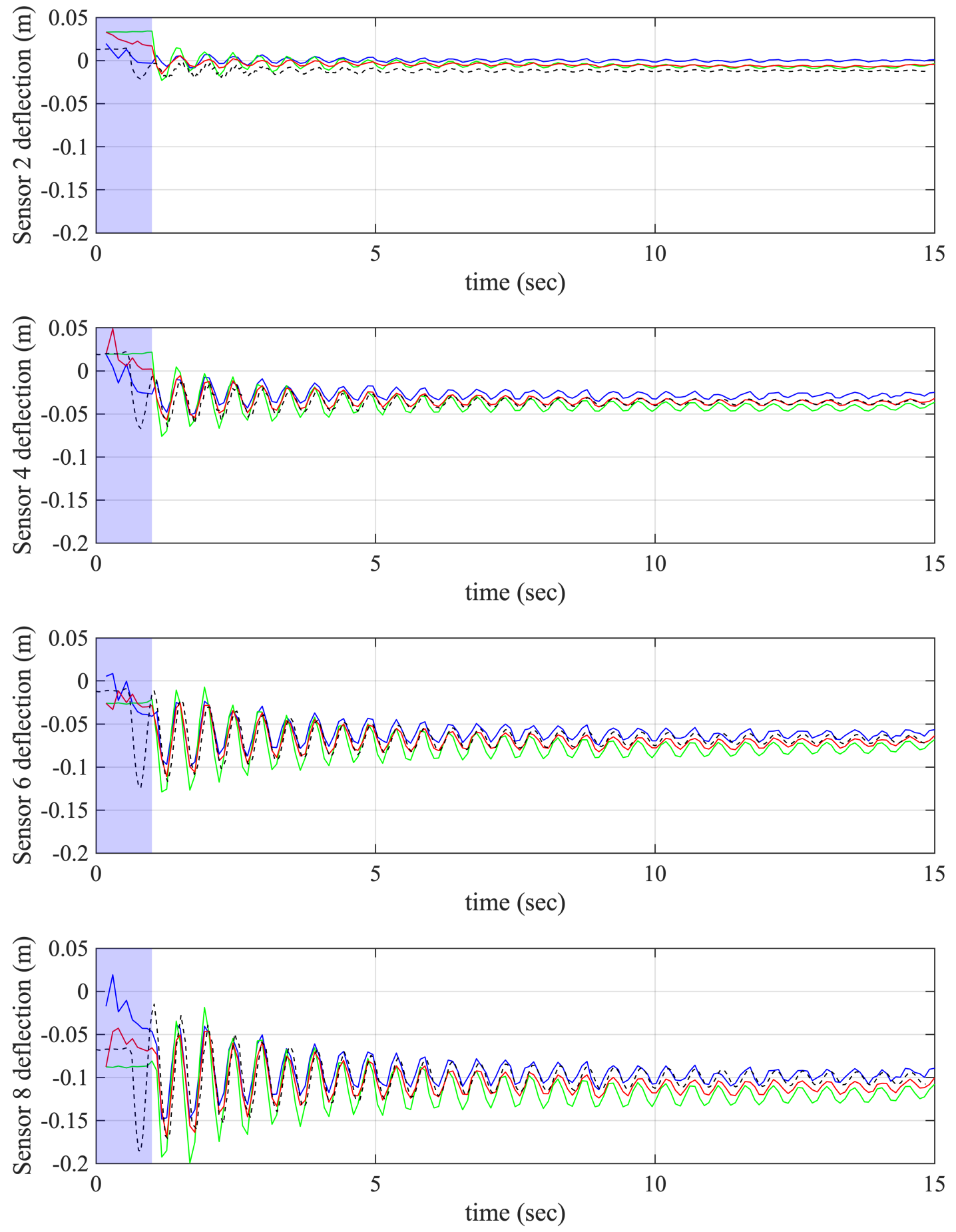

\section{- Model Prediction _ Kalman Filter Estimate \\ Sensor Measurement - - - - - - - Camera Measurement}

Figure 6.7: Initial Conditions: S-Shape Bending, No Twist, Trailing Edge Sensor Row 


\subsubsection{Free Vibration with combined bending and twist initial condition}

The finite element model for the Kalman filter was modified by manipulating the material properties for the FEM model to best reflect the actual deflection. The covariance matrices were also modified to shift weight from the prediction to the measurement and the final experiment was conducted. The test was setup to incorporate initial conditions of combined twist and bending deformation as illustrated in figure Figure 6.8.

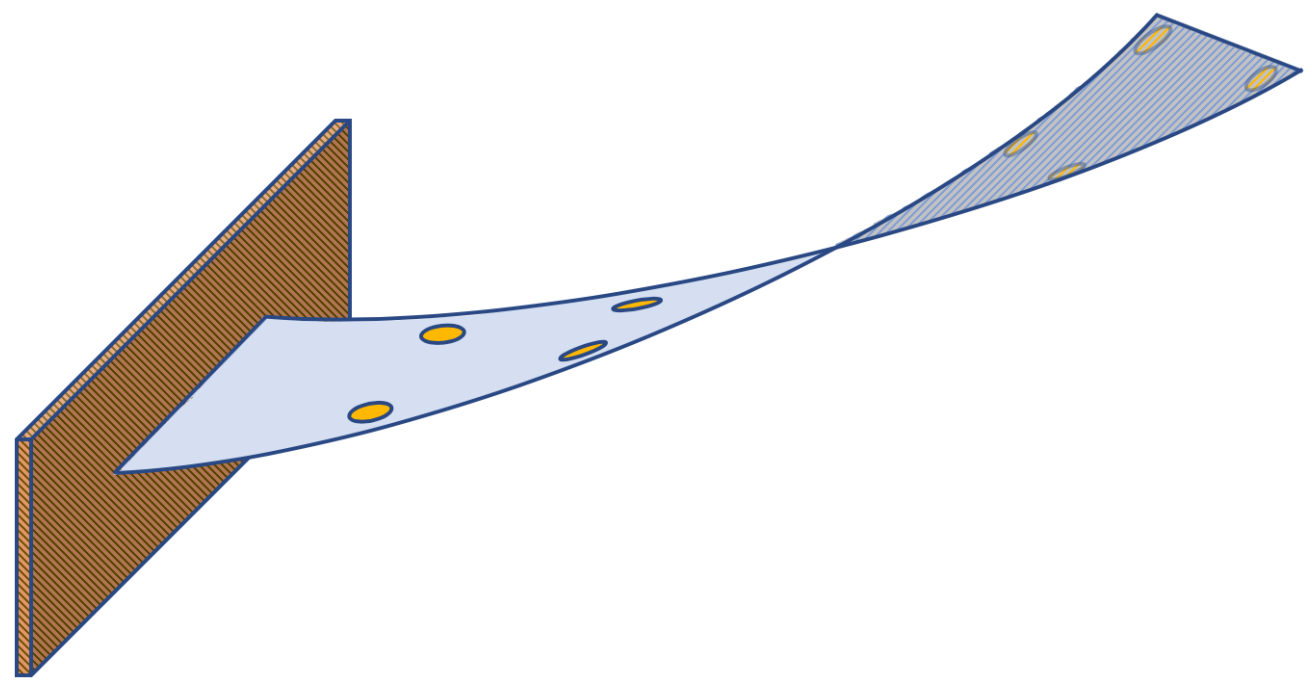

Figure 6.8: Twist Coupled with Bending

The collected data in Figures 6.9 and 6.10 shows the prediction, measurement and state estimate as well as the true deflection for the most complex case tested, that of a coupled twist and bending deformation for an initial condition. The structure was deformed accordingly and subsequently released to oscillate freely. For the data shown, no corrective calibration offset was required. The data provides explicit information on the vertical deformation of the test section but also offers an implicit approximation of the twist. The state estimate shows favorable estimation of the true deflection. The slight over shoot of the measurement that was observed in prior tests reappears in this experiment, however that prediction shows significantly more accuracy when compared to the true deformation. There is small phase lag visible between the measurement and remaining lines for prediction, estimate and actual deflection data. 

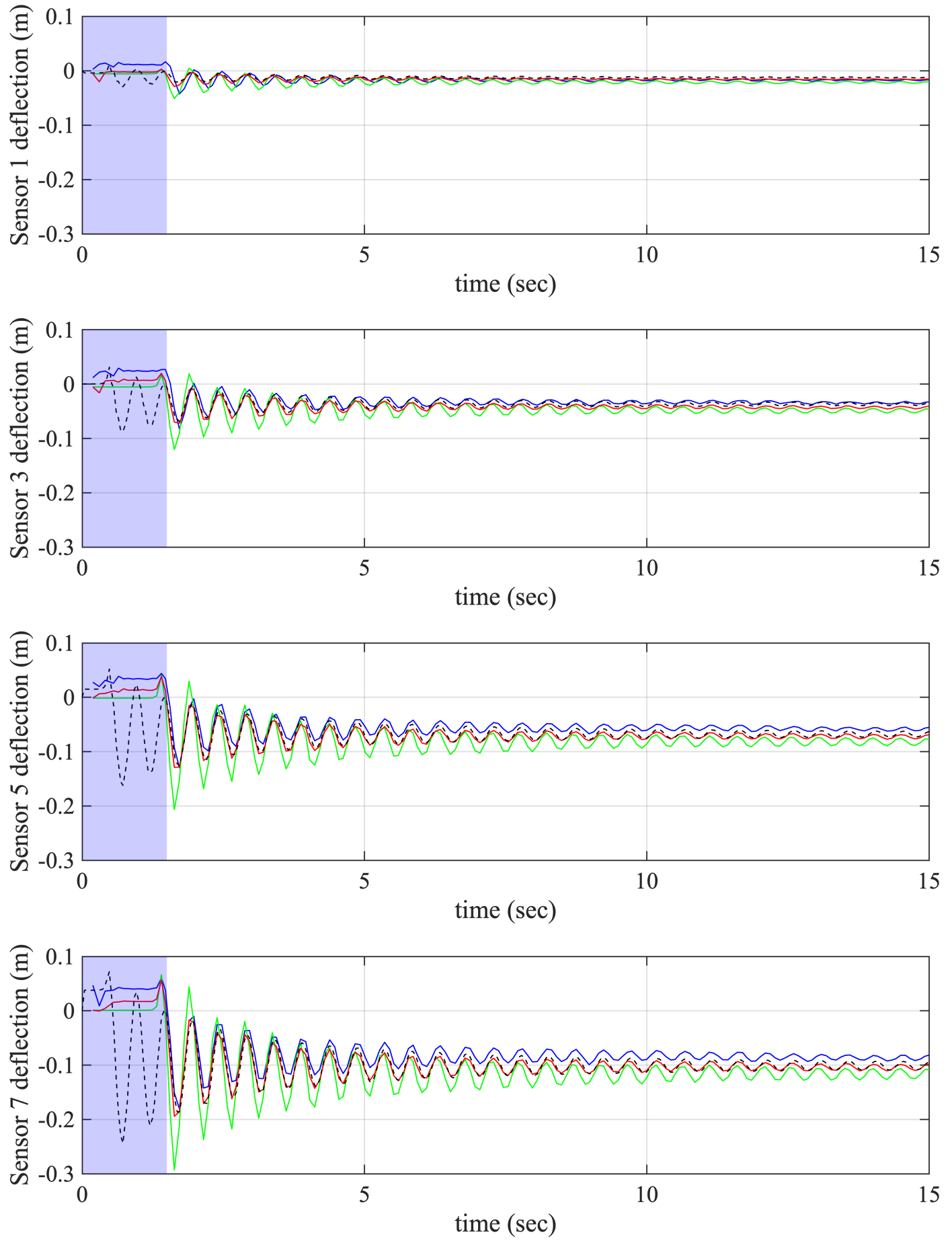

\section{Model Prediction _ Kalman Filter Estimate \\ Sensor Measurement - - - - - - - Camera Measurement}

Figure 6.9: Initial Conditions: Twist Coupled with Bending, Leading Edge Sensor Row 

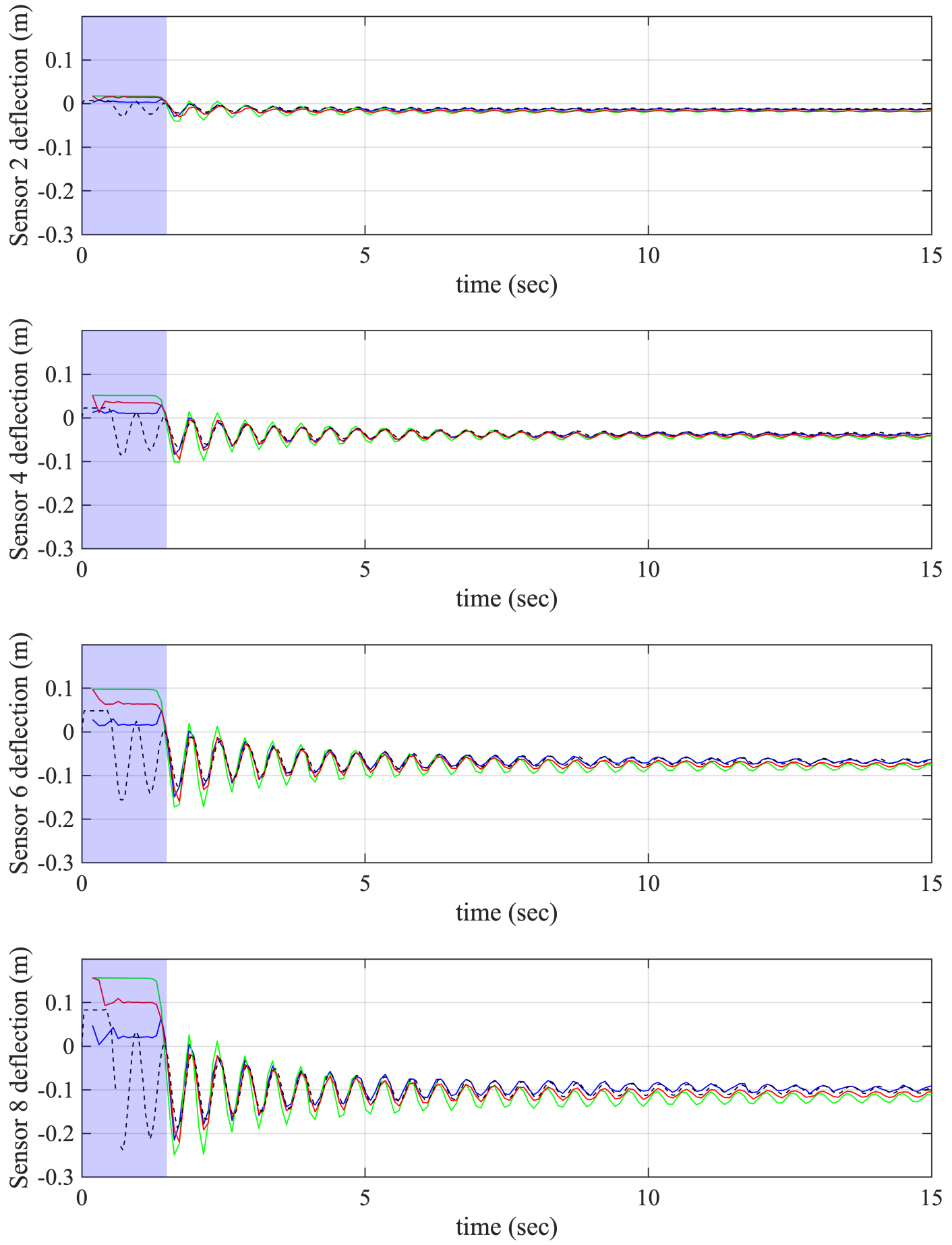

Model Prediction Kalman Filter Estimate

Sensor Measurement -....-.- Camera Measurement

Figure 6.10: Initial Conditions: Twist Coupled with Bending, Trailing Edge Sensor Row 
Table 6.3 shows the root mean square error for the final experiment of combined twist and bending initial condition. Most observations regarding the accuracy are very comparable to those made for previously discussed experiments and show acceptable performance characteristics. The measurement data shows to be significantly more accurate than the prediction suggesting further adjustments to the process and measurement covariance matrices in accordance with the presented results for any future experimentation. The estimate shows an increasing root mean square error from root to tip which is expected since the error of displacement approximation gets progressively larger.

TABLE 6.3

RMS ERROR TABLE FOR COMBINED BENDING AND TWIST

\begin{tabular}{|c|c|c|c|}
\hline Sensor/Node & Measurement RMSE [m] & Prediction RMSE [m] & Estimate RMSE [m] \\
\hline Sensor 1 & 0.0096 & 0.0055 & 0.0040 \\
\hline Sensor 2 & 0.0066 & 0.0025 & 0.0064 \\
\hline Sensor 3 & 0.0130 & 0.0053 & 0.0080 \\
\hline Sensor 4 & 0.0106 & 0.0057 & 0.0082 \\
\hline Sensor 5 & 0.0178 & 0.0124 & 0.0107 \\
\hline Sensor 6 & 0.0173 & 0.0095 & 0.0130 \\
\hline Sensor 7 & 0.0215 & 0.0193 & 0.0143 \\
\hline Sensor 8 & 0.0243 & 0.0141 & 0.0176 \\
\hline Avg. RMSE & $\mathbf{0 . 0 1 5 1}$ & $\mathbf{0 . 0 0 9 3}$ & $\mathbf{0 . 0 1 0 3}$ \\
\hline
\end{tabular}

\subsection{Wind Tunnel: Dynamic Response - Results}

During the testing in the wind tunnel several observations were made with respect to the previously discussed structural lab testing. When introducing an airflow to the flat plate structure a high frequency vibration started forming even at low angles of attack. This phenomenon can likely be attributed to the airflow around the sensors and causes adverse sensor drift. Most of this effect was mitigated by lowering the bandwidth and load factor range within the hardware library and driver of the IMU sensors to $31.25 \mathrm{~Hz}$ and $2 \mathrm{~g}$ receptively in exchange for more accurate readings. However, some drift reappeared during certain test conditions. As discussed in the previous chapter a hollow foam airfoil was installed to enclose the flat plate allowing for a smoother 
airflow. Nevertheless some high frequency vibration was still encountered when the wing was approaching the stall region. This vibration frequency itself is too high to be accurately captured by either the state estimator or the optical validation system. However, in a post-test analysis of video footage taken during the wind tunnel experiment, this vibration was found to be $40 \mathrm{~Hz}$ and higher which poses am additional limitation. During this test the state estimator was started prior to starting up the wind tunnel or validation system. Figure 6.11 shows the entire state estimation including the time interval where the estimation is validated by using the camera equipment as well the region where drift starts to occur. Inspecting a close-up of this region Figure 6.12 reveals a changing trend in oscillatory behavior. The region coincides with an increase in angle of attack commanded during the experiment which can be seen in Figure 6.11 between 84 and 96 seconds, enclosed by the cyan box. The drift most likely begins to occur because the IMU sensor does not have sufficient time to reset its calibration values.

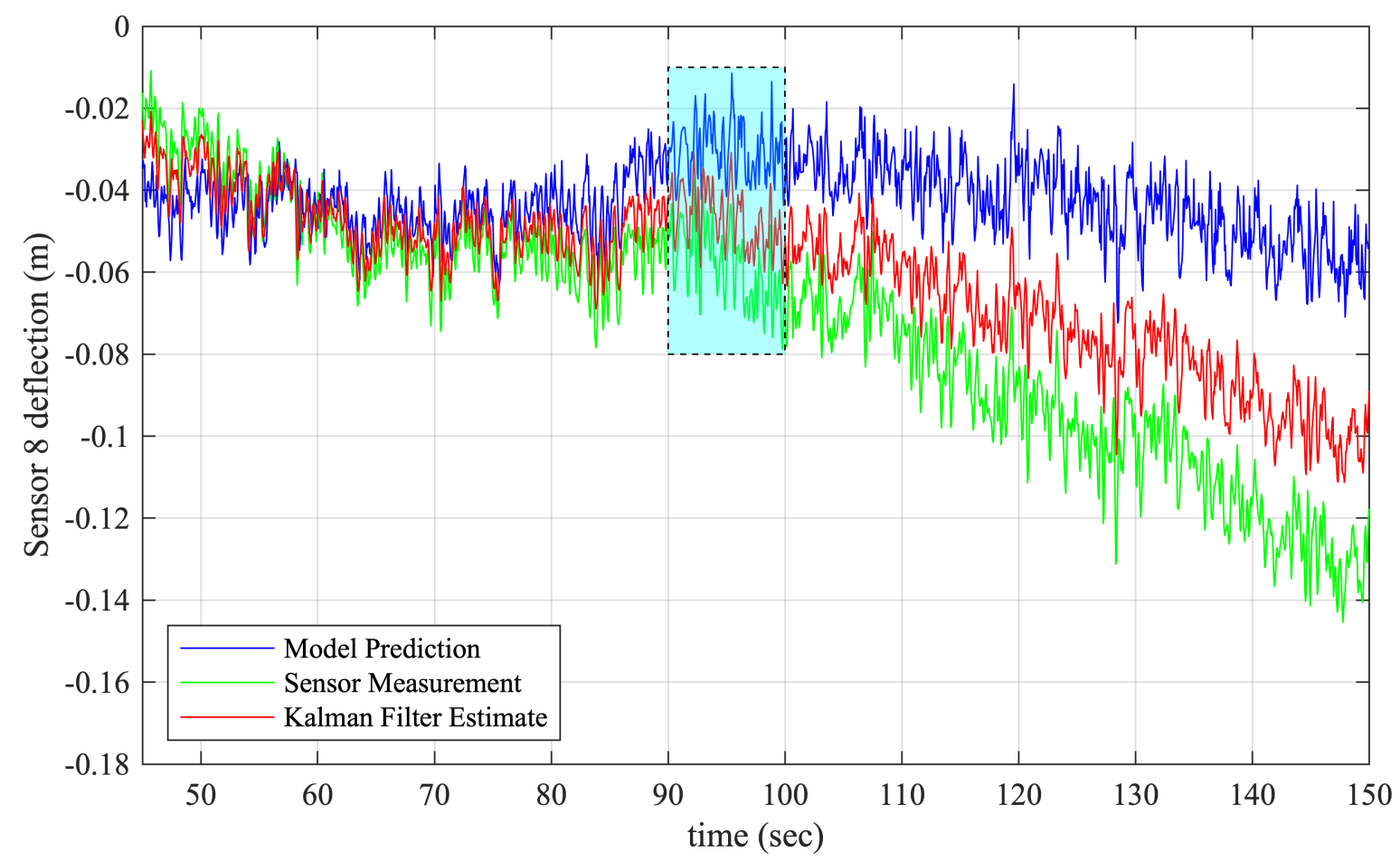

Figure 6.11: Kalman filter performance over an extended periods of time 


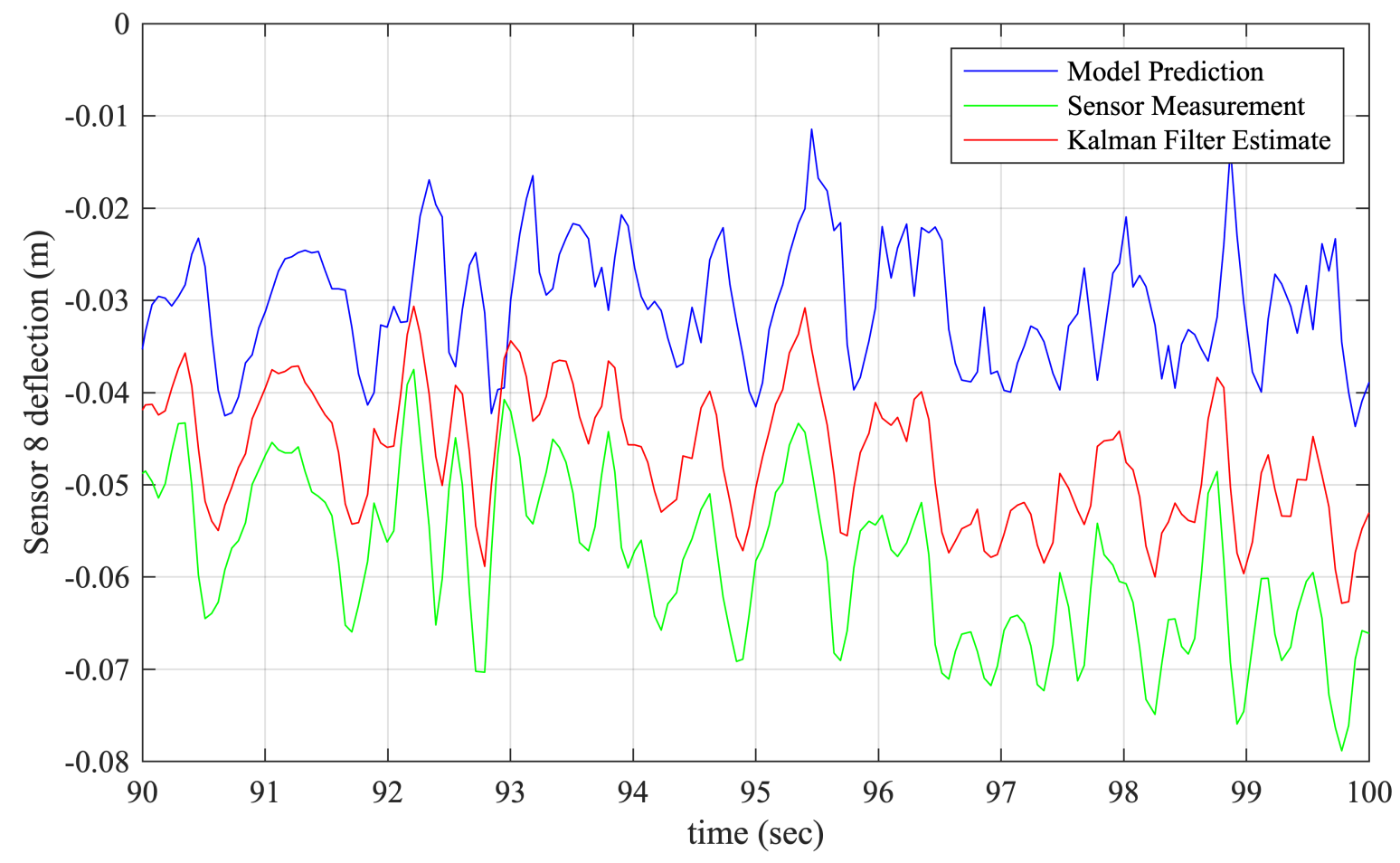

Figure 6.12: Region Of Drift Onset

Figures 6.13 and 6.14 show the deflection data respective of the leading edge and trailing edge sensors for the flat plate which was enclosed in a foam airfoil Figure 5.8. The experiment was conducted at 15 degree AOA and 2.5 PSF (approximately $45 \mathrm{ft} / \mathrm{s}$ ). Inspection of the data shows a greater baseline deflection of the leading edge compared to the trailing edge. This difference corresponds to the initial angle of attack of $15^{\circ}$. A constant post process calibration bias was applied. The state prediction (blue graph) shows significant process noise for all nodes when compared to the deflection measurement or actual deflection. This is attributed to the fact that the finite element model for the flat plate no longer fully holds whit the airfoil enclosure installed. During in the post-data processing covariance matrices $\mathrm{Q}$ and $\mathrm{R}$ were fine-tuned such that more weight was given to the measurement data than the prediction. The Kalman filter was subsequently run again with the offline data which the results reflect. The reader may refer to the appendix for details. The Kalman filter state estimator filters most of the noise. Table 6.4 shows the average difference between the state estimate and the actual deflection of each node. 

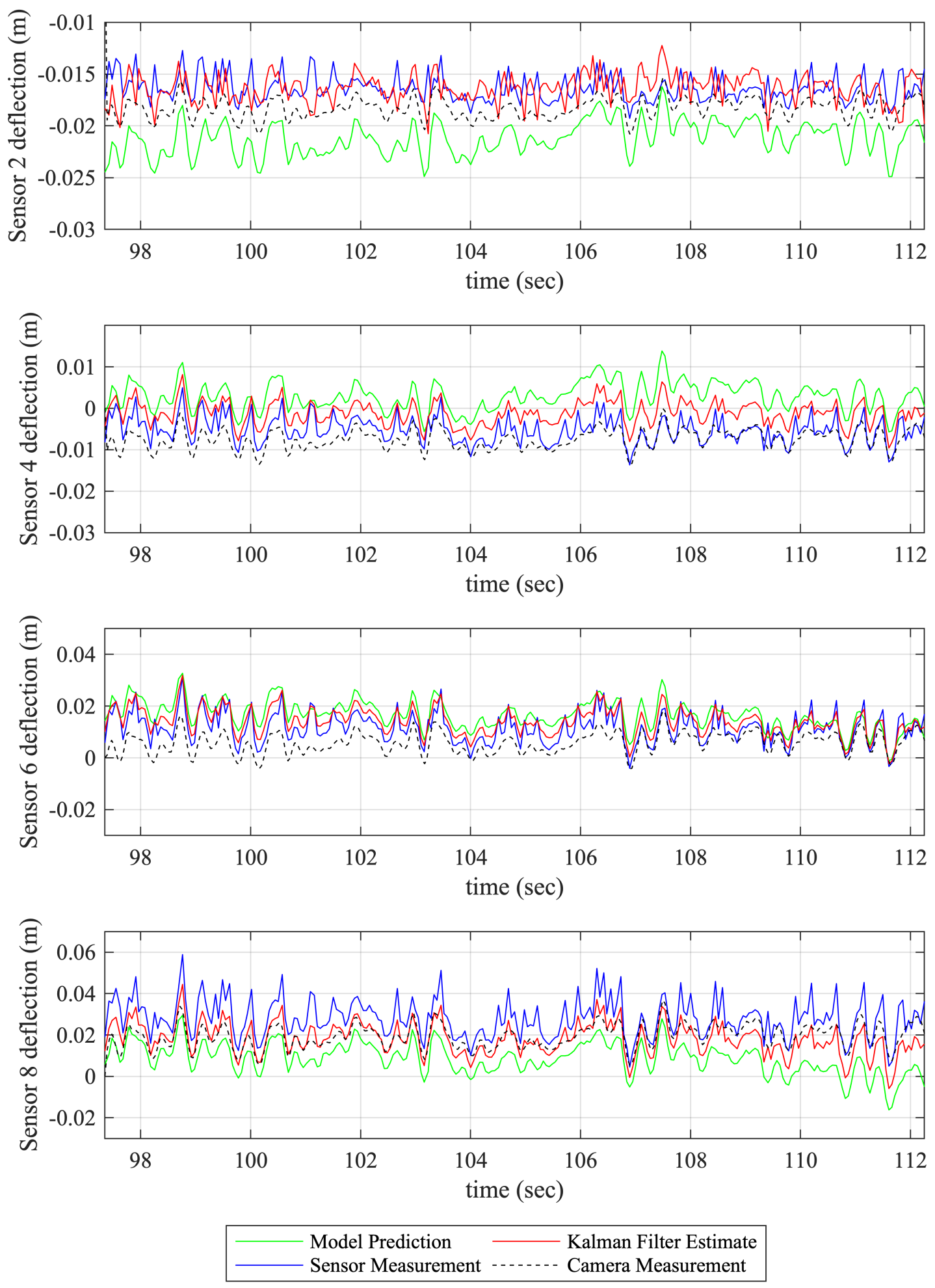

Figure 6.13: Wind Tunnel Test: 2.5 PSF, $15^{\circ}$ AOA:Leading Edge Sensor Row 

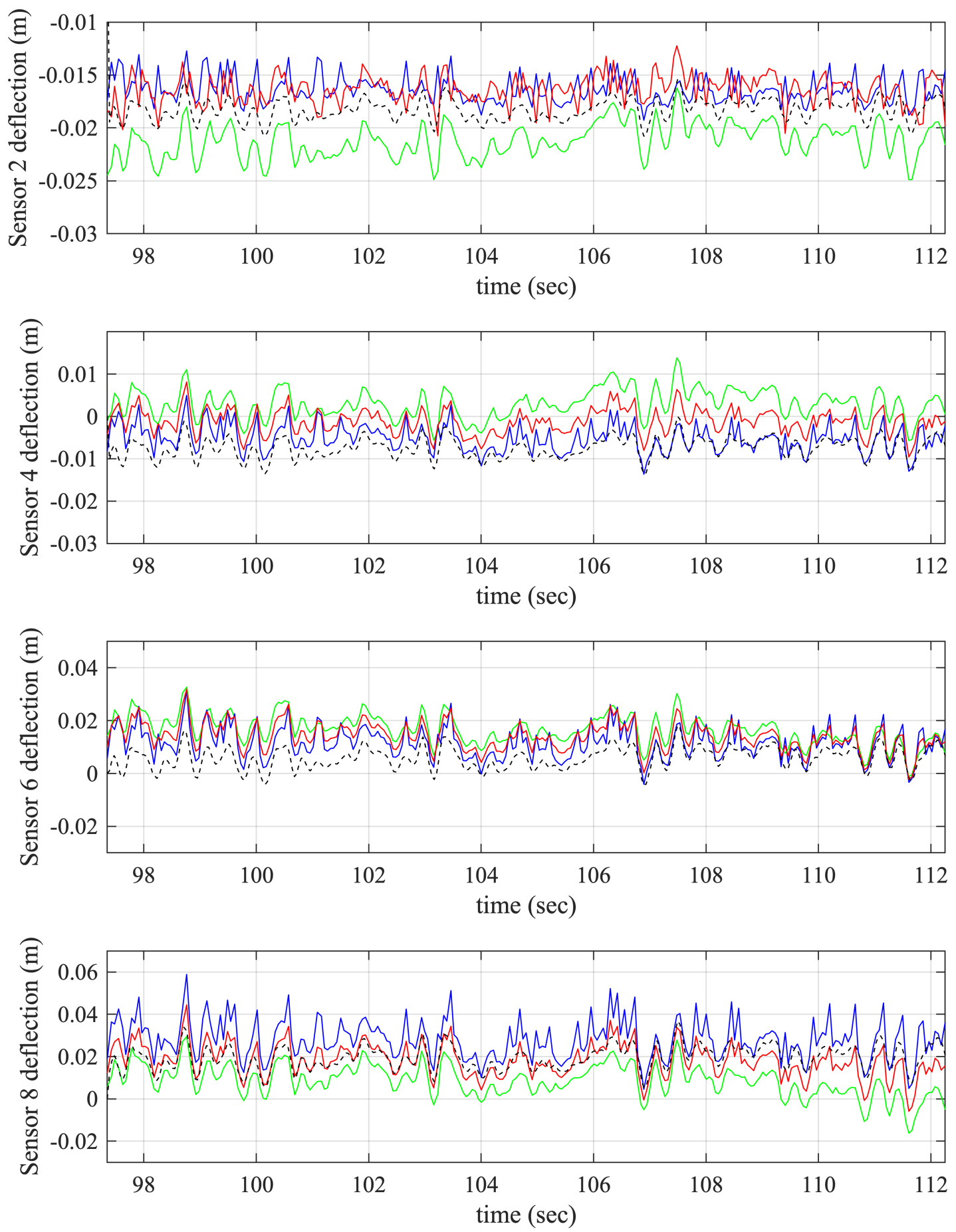

\begin{tabular}{|c|c|}
\hline $\begin{array}{l}\text { Model Prediction } \\
\text { Sensor Measurement }\end{array}$ & $\begin{array}{l}\text { Kalman Filter Estimate } \\
\text { Camera Measurement }\end{array}$ \\
\hline
\end{tabular}

Figure 6.14: Wind Tunnel Test: 2.5 PSF, $15^{\circ}$ AOA: Trailing Edge Sensor Row 
Table 6.4 show the RMSE for the wind tunnel test. as seen in previous experiments the error increases from wing root to wing tip. This occurs because the error is cumulative outwards due to the deflection approximation between every sensor. Will the plot Figure 6.14 show significantly more noise, mostly due to the vibration, the root mean square error indicates desirable performance characteristics of the system. Overall, the results suggest that the performance of the Kalman state estimator is satisfactory. Chapter 7 summarizes the research efforts, the process and the results in a concluding statement.

TABLE 6.4

RMS ERROR TABLE FOR WIND TUNNEL TEST

\begin{tabular}{|c|c|c|c|}
\hline Sensor/Node & Measurement RMSE & Prediction RMSE & Estimate RMSE \\
\hline Sensor 1 & 0.0015 & 0.0048 & 0.0011 \\
\hline Sensor 2 & 0.0029 & 0.0219 & 0.0019 \\
\hline Sensor 3 & 0.0118 & 0.0054 & 0.0009 \\
\hline Sensor 4 & 0.0105 & 0.0063 & 0.0023 \\
\hline Sensor 5 & 0.0145 & 0.0101 & 0.0060 \\
\hline Sensor 6 & 0.0108 & 0.0082 & 0.0058 \\
\hline Sensor 7 & 0.0125 & 0.0035 & 0.0197 \\
\hline Sensor 8 & 0.0107 & 0.0010 & 0.0088 \\
\hline Avg. RMSE & $\mathbf{0 . 0 0 9 2}$ & $\mathbf{0 . 0 0 5 3}$ & $\mathbf{0 . 0 0 8 6}$ \\
\hline
\end{tabular}

A conclusion is drawn in Chapter 7 and opportunities for future research, reflecting on the results that were provided in this chapter, are also discussed. 


\section{CHAPTER 7}

\section{CONCLUSION}

The work described within this document provides a detailed account of research efforts directed towards the design and the experimental testing of an inertial sensor system which can be used to quantify deformation of an aircraft structure in real time. The system involved the implementation of a Kalman filter state estimator. A detailed literature review illustrated relevant research conducted by others, whose work included various approaches to quantify displacement but also discuss methods which would prove integral to the direction of this project. Various approaches were considered to include electromagnetic ranging and interferometry which were described in detail. Most of these methods however proved to be inefficient or for other reasons not feasible. By far the most promising method was the implementation of microelectronic mechanical sensors (MEMS sensors). A variety of such sensors was analyzed and evaluated for their performance. A kinematic approach was taken and preliminary system was designed to detect the displacement of a pivoting beam and subsequently a bending and twisting structure. Preliminary testing revealed promising results of the kinematic approach but also limitations which were discussed in part 1 of the results chapter (chapter 4). A structural dynamics model to capture the deformation of a structure while in motion needed to be included. Knowledge gained from the preliminary testing led to an advanced method which was based on the notion of combining an analytical finite element model with real time sensor measurement. Extensive bench and wind tunnel testing was conducted. Part 2 of the results section details the performance characteristics and outlines limitations of sensors and other components of the system.Based on the findings a central conclusion can be drawn: The described system consisting of an analytical finite element model fused with low cost, 'off the shelf' microelectronic inertial sensors is capable of providing sufficiently accurate and timely translational and rotational motion data to deduce information on the structural deformation. Results suggest that even at small changes in angular velocity or linear acceleration during high frequency vibration encounters are detectable. 
The system design in its current developmental stage is restricted as to the type of deformation it can sense. This can be largely attributed to the structural model which is limited to vertical displacement and angles $\phi$ and $\theta$. A more advanced and elaborate finite element model should be considered and should expand on the aerodynamic aspects to include a more realistic lift distribution and drag effects for various airfoils. Structurally such a model should account for transverse shear loads and non homogeneous material composition. The sensors that are currently used exhibit significant limitations with respect to adverse drift. While both accuracy and range are sufficient, a sensor model that is more robust to high frequency mechanical vibrations will likely improve the performance in terms of long term accuracy and speed significantly. Last, in an effort to capture and model non-typical deformations or those which exceed the capabilities of a conventional state estimator or the applied structural model, the application of a neural network could be explored. The neural network can be trained by querying sensors across the wing structure during various deformations. The collected raw or preprocessed inertial data will be mapped to the current deformation. Over time the network will associate and correlate specific sensor readings with the respective deflections. 
BIBLIOGRAPHY 


\section{BIBLIOGRAPHY}

[1] Nguyen, N. T., Ting, E., Chaparro, D., Drew, M. C., and Swei, S. S.-M., "Multi-Objective Flight Control for Drag Minimization and Load Alleviation of High-Aspect Ratio Flexible Wing Aircraft," 58th AIAA/ASCE/AHS/ASC Structures, Structural Dynamics, and Materials Conference, edited by, AIAA SciTech Forum, Vol. , American Institute of Aeronautics and Astronautics, 2017. doi:doi:10.2514/6.2017-158910.2514/6.2017-1589, URL https:// doi.org/10.2514/6.2017-1589.

[2] Frost, S. A., Wright, C., and Khan, M. A., "Biomimetic optical sensor for real-time aircraft wing deflection measurement," AIAA Guidance, Navigation, and Control Conference, edited by , AIAA SciTech Forum, Vol. , American Institute of Aeronautics and Astronautics, 2015. doi:doi:10.2514/6.2015-008310.2514/6.2015-0083, URL https://doi.org/10. $2514 / 6.2015-0083$.

[3] Grigorie, T., Popov, A., and Botez, R., "Design and experimental validation of a control system for a morphing wing," AIAA Atmospheric flight mechanics conference, 2012, p. 4639.

[4] Popov, A. V., Grigorie, L. T., Botez, R. M., Mamou, M., and Mebarki, Y., "Closed-loop control validation of a morphing wing using wind tunnel tests," Journal of Aircraft, Vol. 47, No. 4, 2010, pp. 1309-1317.

[5] Popov, A. V., Labib, M., Fays, J., and Botez, R. M., "Closed-loop control simulations on a morphing wing," Journal of Aircraft, Vol. 45, No. 5, 2008, pp. 1794-1803.

[6] Menon, A. N., Chakravarthy, A., and Nguyen, N. T., "Adaptive Control for Hybrid PDE Models Inspired from Morphing Aircraft," 2018 AIAA Guidance, Navigation, and Control Conference, 2018, p. 0872.

[7] Ferrier, Y., Nguyen, N. T., and Ting, E., "Real-Time Adaptive Least-Squares Drag Minimization for Performance Adaptive Aeroelastic Wing," 34th AIAA Applied Aerodynamics Conference, 2016, p. 3567.

[8] Menon, A., Chakravarthy, A., Steck, J. E., and Nguyen, N. T., "Decentralized Optimal Adaptive Control Architectures for the VCCTEF Aircraft," 58th AIAA/ASCE/AHS/ASC Structures, Structural Dynamics, and Materials Conference, 2017, p. 1590.

[9] Nguyen, N., and Urnes, J., "Aeroelastic modeling of elastically shaped aircraft concept via wing shaping control for drag reduction," AIAA Atmospheric Flight Mechanics Conference, 2012, p. 4642.

[10] Nguyen, N. T., and Swei, S. S.-M., "Adaptive Linear Quadratic Gaussian Optimal Control Modification for Flutter Suppression of Adaptive Wing," AIAA Infotech@ Aerospace, Vol., edited by , 2015, p. 0118. 
[11] Urnes, J., and Nguyen, N., "A mission adaptive variable camber flap control system to optimize high lift and cruise lift to drag ratios of future $\mathrm{n}+3$ transport aircraft," 51st AIAA Aerospace Sciences Meeting including the New Horizons Forum and Aerospace Exposition, 2013, p. 214.

[12] Sofla, A., Meguid, S., Tan, K., and Yeo, W., "Shape morphing of aircraft wing: Status and challenges," Materials \& Design, Vol. 31, No. 3, 2010, pp. 1284-1292.

[13] Vos, R., Breuker, R. D., Barrett, R. M., and Tiso, P., "Morphing Wing Flight Control Via Postbuckled Precompressed Piezoelectric Actuators," Journal of Aircraft, Vol. 44, No. 4, 2007, pp. 1060-1068. doi:10.2514/1.21292, URL https://doi.org/10.2514/1. 21292.

[14] Draai, R. K. v. d., Schinkel, R. P. M. v., and Telesca, A., "A new approach to measuring model deflection," ICIASF 99. 18th International Congress on Instrumentation in Aerospace Simulation Facilities. Record (Cat. No.99CH37025), 1999, pp. 33/1-33/7. doi:10.1109/ ICIASF.1999.827173.

[15] Veerman, H. P. J., Kannemans, H., and Jentink, H. W., "Highly Accurate Aircraft InFlight Wing Deformation Measurements Based on Image Correlation," Advanced InFlight Measurement Techniques, edited by , Boden, F., Lawson, N., Jentink, H. W., and Kompenhans, J. (eds.), Springer Berlin Heidelberg, Vol. , Berlin, Heidelberg, 2013, pp. 15-32. doi:10.1007/978-3-642-34738-2_3, URL https://doi.org/10.1007/ 978-3-642-34738-2_3.

[16] Davis, J. D., "Measurement of Flexible Wing Deformations in Flight," Master's thesis, 2006.

[17] Dixon, P. B., Starling, D. J., Jordan, A. N., and Howell, J. C., "Ultrasensitive Beam Deflection Measurement via Interferometric Weak Value Amplification," Physical Review Letters, Vol. 102, No. 17, 2009, p. 173601. URL/https://link.aps.org/doi/10 . 1103 /PhysRevLett.102.173601.

[18] Deangelis, V. M., "In-flight deflection measurement of the HiMAT aeroelastically tailored wing," Journal of Aircraft, Vol. 19, No. 12, 1982, pp. 1088-1094. doi:10.2514/3.44816, URL https://doi.org/10.2514/3.44816.

[19] Deets, D. A., DeAngelis, V. M., and Lux, D. P., "HiMAT flight program: Test results and program assessment overview," 1986.

[20] Cusano, A., Capoluongo, P., Campopiano, S., Cutolo, A., Giordano, M., Felli, F., Paolozzi, A., and Caponero, M., "Experimental modal analysis of an aircraft model wing by embedded fiber Bragg grating sensors," IEEE Sensors Journal, Vol. 6, No. 1, 2006, pp. 67-77. doi: 10.1109/JSEN.2005.854152.

[21] Richards, L., Parker, A. R., Ko, W. L., and Piazza, A., "Real-time in-flight strain and deflection monitoring with fiber optic sensors," 2008. 
[22] Bogert, P., Haugse, E., and Gehrki, R., "Structural Shape Identification from Experimental Strains Using a Modal Transformation Technique," 44th AIAA/ASME/ASCE/AHS/ASC Structures, Structural Dynamics, and Materials Conference, edited by , Structures, Structural Dynamics, and Materials and Co-located Conferences, Vol. , American Institute of Aeronautics and Astronautics, 2003. doi:doi:10.2514/6.2003-162610.2514/6.2003-1626, URL https: //doi.org/10.2514/6.2003-1626.

[23] Menon, A., Rafi, M., Schirmer, G., Tran, T., Whitford, J., Yodo, N., Kostogorova-Beller, Y., Steck, J., Chakravarthy, A., and Keshavanarayana, S., "Measurement of wing deflections in flexible aircraft using the sansec smart sensor," IEEE Aerospace and Electronic Systems Magazine, Vol. 32, No. 10, 2017, pp. 14-23. doi:10.1109/MAES.2017.160133.

[24] Menon, A., Whitford, J., Kostogorova-Beller, Y., Steck, J. E., and Chakravarthy, A., "Flexible Deflection Measurements using the SansEC Smart Sensor," 58th AIAA/ASCE/AHS/ASC Structures, Structural Dynamics, and Materials Conference, 2017, p. 1586.

[25] Bahl, P., Padmanabhan, V. N., et al., "RADAR: An in-building RF-based user location and tracking system," IEEE infocom, Vol. 2, INSTITUTE OF ELECTRICAL ENGINEERS INC (IEEE), 2000, pp. 775-784.

[26] Gong, X., Liu, H., and Yan, X.-G., "Deformation Measuring Methods Based on Inertial Sensors for Airborne Distributed POS," International Journal of Aerospace Engineering, Vol. 2017, 2017, p. 12. doi:10.1155/2017/9343215, URL https://doi.org/10.1155/ $2017 / 9343215$.

[27] Kowalczuk, Z., and Merta, T., "Evaluating the position of a mobile robot using accelerometer data," Advanced and Intelligent Computations in Diagnosis and Control, Springer, 2016, pp. 131-143.

[28] Lanzisera, S. M., and Pister, K., "RF ranging for location awareness," Thesis, 2009.

[29] Bauer, A. O., Erskine, R., and Herold, K., Funkpeilung als alliierte Waffe gegen deutsche U-Boote 1939-1945: wie Schwächen und Versäumnisse bei der Funkführung der U-Boote zum Ausgang der" Schlacht im Atlantik" beigetragen haben, Liebich Funk, 1997.

[30] Woodard, S. E., "SansEC sensing technology. A new tool for designing space systems and components," 2011 Aerospace Conference, IEEE, 2011, pp. 1-11.

[31] Kalman, R. E., "A new approach to linear filtering and prediction problems," Journal of basic Engineering, Vol. 82, No. 1, 1960, pp. 35-45.

[32] Gilbert, H. B., Celik, O., and O’Malley, M. K., "Long-term double integration of acceleration for position sensing and frequency domain system identification," Advanced Intelligent Mechatronics (AIM), 2010 IEEE/ASME International Conference on, IEEE, 2010, pp. 453458.

[33] Reddy, J., An introduction to the finite element method, Vol. 1221, McGraw-Hill New York, USA, 2013. 
[34] Rao, S. S., Vibration of continuous systems, Wiley, 2019.

[35] Bhavsar, N. R., Shinde, H., and Bhat, M., "Determination of Mechanical Properties of PCB," International Journal on Mechanical Engineering and Robotics, Vol. 2, No. 4, 2014, pp. 23-27. 
APPENDICES 


\section{APPENDIX A}

\section{DATA SHEETS}

\section{Technical Data}

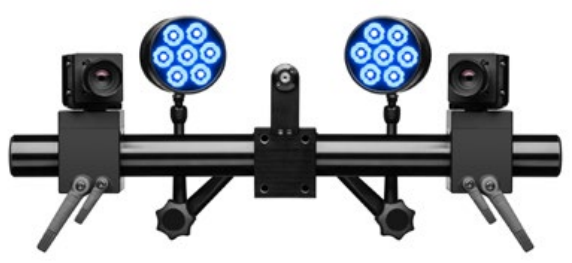

\section{ARAMIS Adjustable}

$2.3 \mathrm{M}$

\begin{tabular}{l|l}
\hline Camera Sensor & CMOS \\
\hline Camera Resolution & $1936 \times 1216$ Pixel \\
\hline Internal RAM & - \\
\hline Frame Rate & $130 \mathrm{fps} @$ full resolution \\
& $240 \mathrm{fps} @ 1 / 2$ image height \\
& $450 \mathrm{fps} @ 1 / 4$ image height \\
\hline Illumination & Dual Blue Light LED
\end{tabular}

Camera Frame [mm]

Measuring Area [mm]

Control Device

Ambient Conditions

Voltage Range (typical)

Power Consumption

Cable Length

Strain Measuring Range

Strain Measuring Resolution

Specimen Temperature
$500 \mid 800$

$10 \times 6$ up to $4000 \times 2500$

GOM Testing Controller

$+5^{\circ} \mathrm{C}$ to $+40^{\circ} \mathrm{C}$ (non-condensing)

$100-240$ V AC, $50-60 \mathrm{~Hz}$

typically $15 \mathrm{~W}$

maximum $100 \mathrm{~W}$

$10 \mathrm{~m}$

$30 \mathrm{~m}$

$0.005 \%$ up to $>2000 \%$

typ. $-100^{\circ} \mathrm{C}$ up to $+1500^{\circ} \mathrm{C}$
$6 \mathrm{M}$

CCD

$2752 \times 2200$ Pixel

$-$

25 fps@ @ull resolution

44 fps@binning

Dual Blue Light LED

$500 \mid 800$

$10 \times 8$ up to $5000 \times 4000$

GOM Testing Controller

$+5^{\circ} \mathrm{C}$ to $+40^{\circ} \mathrm{C}$ (non-condensing)

$100-240$ V AC, $50-60 \mathrm{~Hz}$

typically $15 \mathrm{~W}$

maximum $100 \mathrm{~W}$

$10 \mathrm{~m}$

$30 \mathrm{~m}$

$0.005 \%$ up to $>2000 \%$

up to $0.005 \%$

typ. $-100^{\circ} \mathrm{C}$ up to $+1500^{\circ} \mathrm{C}$

Figure A.1: Sensor: ARAMIS Adjustable Base 6M (Courtesy of GOM) 


\section{BNO055}

\section{INTELLIGENT ABSOLUTE ORIENTATION SENSOR, 9-AXIS SENSOR FUSION ALL-IN-ONE WINDOWS 8.x COMPLIANT SENSOR HUB}

\section{Basic Description}

Key features:

- Outputs fused sensor data

- 3 sensors in one device

- Small package

- Power Management

- Common voltage supplies

- Digital interface

- Consumer electronics suite

Quaternion, Euler angles, Rotation vector, Linear acceleration, Gravity, Heading an advanced triaxial 16bit gyroscope, a versatile, leading edge triaxial 14bit accelerometer and a full performance geomagnetic sensor

LGA package 28 pins Footprint $3.8 \times 5.2 \mathrm{~mm}^{2}$, height $1.13 \mathrm{~mm}^{2}$ Intelligent Power Management: normal, low power and suspend mode available $\mathrm{V}_{\mathrm{DD}}$ voltage range: $2.4 \mathrm{~V}$ to $3.6 \mathrm{~V}$ HID-I2C (Windows 8 compatible), I² , UART $\mathrm{V}_{\mathrm{DDIO}}$ voltage range: $1.7 \mathrm{~V}$ to $3.6 \mathrm{~V}$ MSL1, RoHS compliant, halogen-free Operating temperature: $-40^{\circ} \mathrm{C} \ldots+85^{\circ} \mathrm{C}$

Key features of integrated sensors:

Accele rometer features

- Programmable functionality

Acceleration ranges $\pm 2 \mathrm{~g} / \pm 4 \mathrm{~g} / \pm 8 \mathrm{~g} / \pm 16 \mathrm{~g}$ Low-pass filter bandwidths $1 \mathrm{kHz}-<8 \mathrm{~Hz}$ Operation modes:

- Normal

- Suspend

- Low power

- Standby

- Deep suspend

- On-chip interrupt controller

Motion-triggered interrupt-signal generation for

- any-motion (slope) detection

- slow or no motion recognition

- high-g detection

Figure A.2: BNO055 Data Sheet (Courtesy of Adafruit Inc.) 

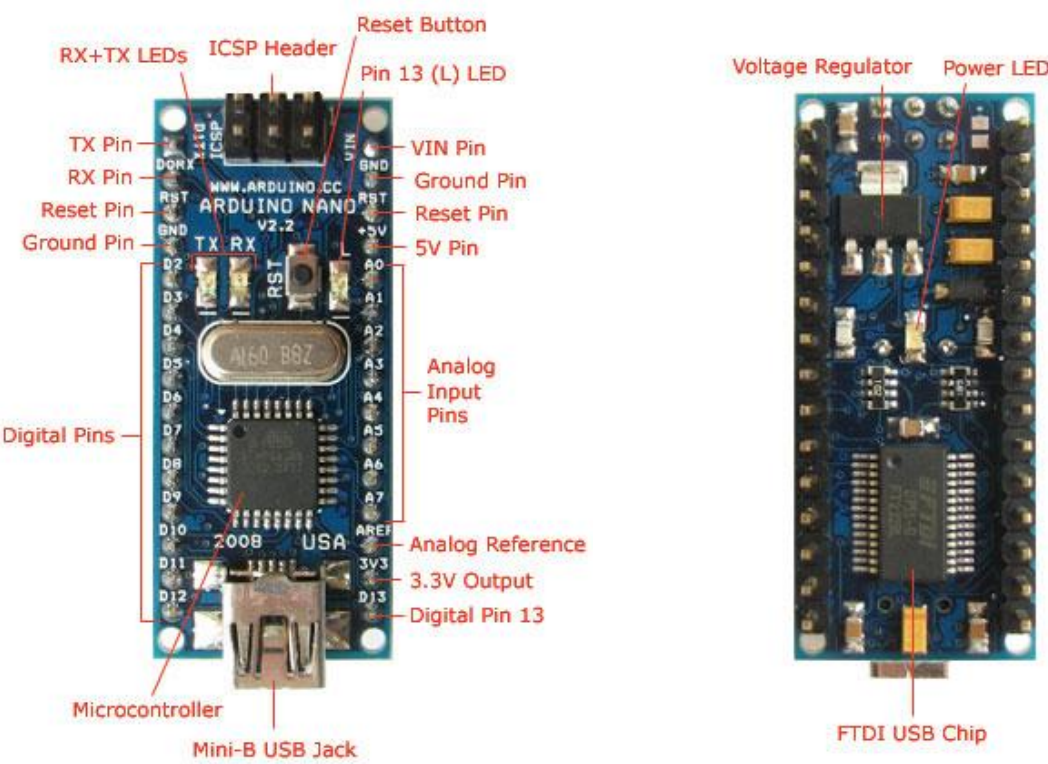

\section{Schematic and Design}

Arduino Nano 3.o (ATmega328): schematic, Eagle files.

Arduino Nano 2.3 (ATmega168): manual (pdf), Eagle files. Note: since the free version of Eagle does not handle more than 2 layers, and this version of the Nano is 4 layers, it is published here unrouted, so users can open and use it in the free version of Eagle.

\section{Specifications:}

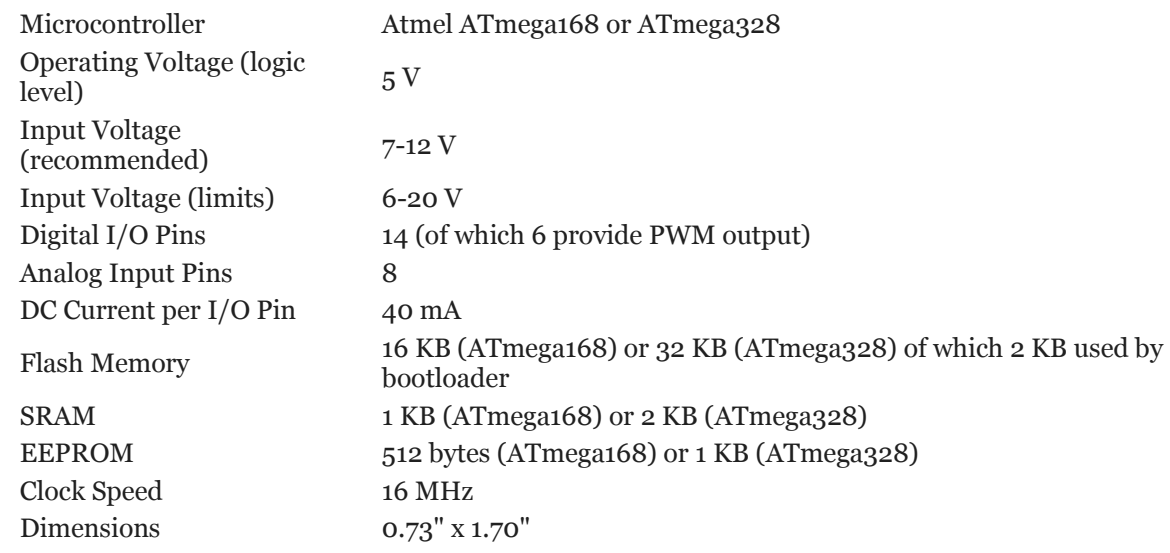

\section{Power:}

The Arduino Nano can be powered via the Mini-B USB connection, 6-20V unregulated external power supply (pin 30), or $5 \mathrm{~V}$ regulated external power supply (pin 27). The power source is automatically selected to the highest voltage source.

Figure A.3: Arduino Nano Data Sheet (Courtesy of element14) 